\title{
Survey of the vascular plants of Sierra Chica, the untouched area of the Paititi Natural Reserve (southeastern Tandilia mountain range, Buenos Aires province, Argentina)
}

\author{
María L. Echeverría, ${ }^{1}$ Sara I. Alonso, ${ }^{1}$ Viviana M. Comparatore ${ }^{2}$ \\ 1 Universidad Nacional de Mar del Plata, Facultad de Ciencias Agrarias, Ruta 226, km 73.5, CC 273, 7620, Balcarce, Buenos Aires, Argentina. \\ Instituto Nacional de Tecnología Agropecuaria, Centro Regional Buenos Aires Sur, Estación Experimental Agropecuaria Balcarce, Argentina. \\ 2 Universidad Nacional de Mar del Plata, Facultad de Ciencias Exactas y Naturales, Funes 3250, CC 7600, Mar del Plata, Buenos Aires, Argentina. \\ Instituto de Investigaciones Marinas y Costeras, CONICET. \\ Corresponding author. E-mail: echeverria.marialis@inta.gob.ar
}

\begin{abstract}
The Paititi Natural Reserve is located in the southeastern part of the Tandilia mountain range (Buenos Aires province, Argentina). With the aim of recording the floristic richness of Sierra Chica, an untouched area of the reserve, all vascular plants were inventoried and categorized by family, status, and life form. Altogether, 364 taxa from 72 families were found, with the most predominant families being Poaceae (20.7\%), Asteraceae (20.1\%), and Fabaceae (5.8\%). We found 95 adventive species, 4 cosmopolitan, and 265 native. The most abundant life forms were hemicryptophytes (33.1\%), therophytes $(30.6 \%)$, and criptophytes $(22.6 \%)$; the therophytes were mostly represented by adventive species. We also found species that were known from Buenos Aires province but not from mountainous areas. Despite being a small area within Argentina, Sierra Chica constitutes a valuable refuge for native flora of the Tandilia mountain range system, which is in danger of being affected by exotic plants.
\end{abstract}

\section{Key words}

Floristic inventory; life form; status; endemism; conservation; threatened species.

Academic editor: Guilherme Dubal dos Santos Seger | Received 2 June 2016 | Accepted 27 August 2017 | Published 15 December 2017

Citation: Echeverría ML, Alonso SI, Comparatore VM (2017) Survey of the vascular plants of Sierra Chica, the untouched area of the Paititi Natural Reserve (southeastern Tandilia mountain range, Buenos Aires province, Argentina). Check List 13 (6): 1003-1036. https://doi.org/10.15560/13.6.1003

\section{Introduction}

In the last decades, agricultural intensification (Benton et al. 2003) and urban and rural population growth (MEA 2005) have been the main causes of biodiversity decline in agricultural landscapes (Krebs et al. 1999). Fire, habitat fragmentation, introduction of exotic species, and high rates of agrochemical application have generated negative impacts on different habitats, and therefore, on the diversity of plants and animals (Frangi et al. 1980;
Tscharntke et al. 2005). The province of Buenos Aires, located in the east-central region of Argentina, due to the advance of the farming frontier, does not escape this phenomenon (Bilenca and Miñarro 2004, Paruelo et al. 2005, Viglizzo et al. 2011). However, the Tandilia and Ventania mountain range systems disrupt the typical plain landscape of the province, limiting the extent of agricultural fields, and acting as biodiversity reservoirs (Fig. 1). The rocky outcrops are known hotbeds of biodiversity that allow refuge for native, endemic, and threatened species, 


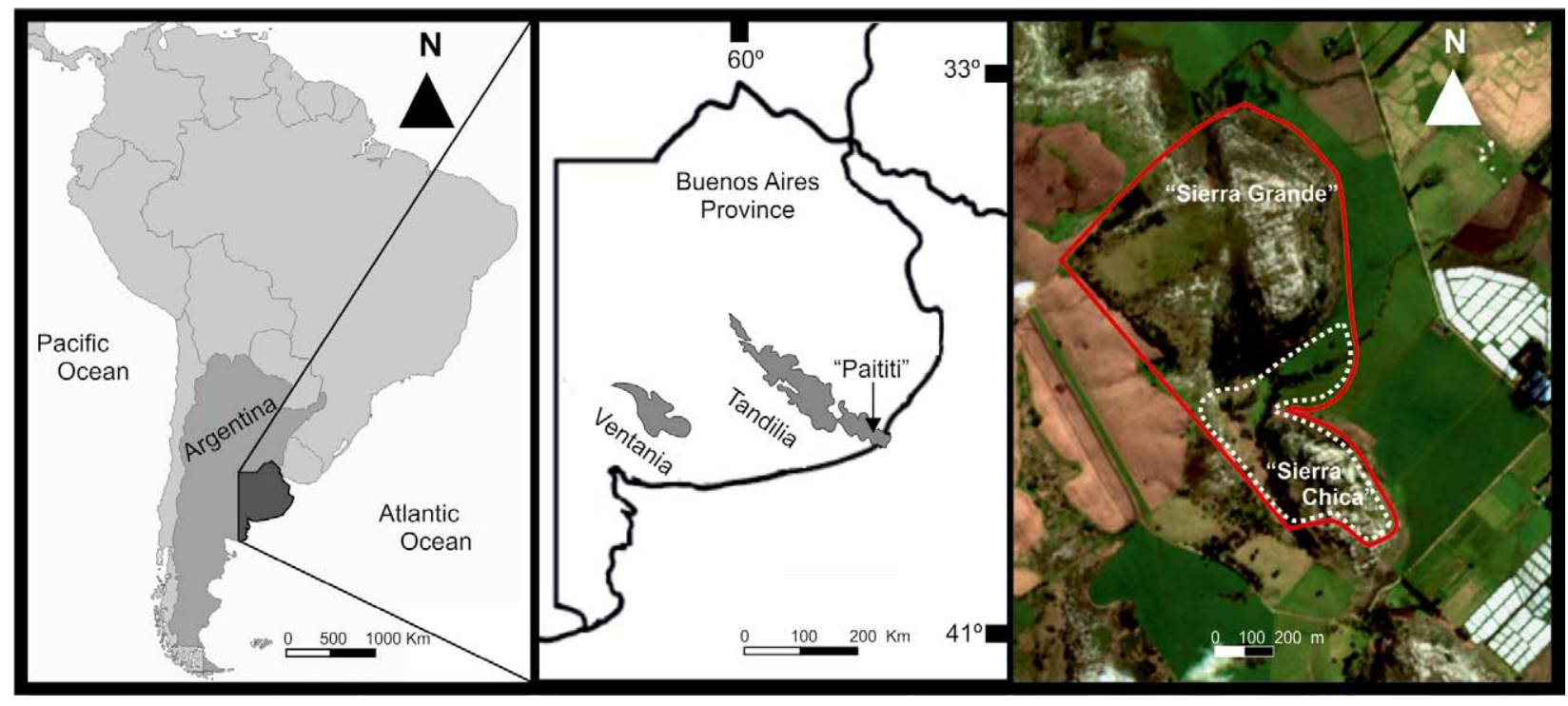

Figure 1. Location of Paititi Natural Reserve, Tandilia System of Mountain Range, Buenos Aires Province, Argentina. The area surrounded by a red line corresponds to the Paititi Natural Reserve while the area surrounded with a dotted white line corresponds to "Sierra Chica". Satellite image obtained from Sentinel Playground (Sentinel Playground, 2017).

and contribute to the conservation of these species (OPDS 2011, Cantero et al. 2014, Kristensen et al. 2014). In this regard, Bertonatti and Corcuera (2000) considered the mountain ranges as areas of outstanding biodiversity, and Bilenca and Miñarro (2004) highlighted that in Buenos Aires province, $67 \%$ of the vascular species with conservation priority can be found in Tandilia and Ventania mountain range systems.

The available information on the flora of the Tandilia system, (Frangi 1975, Valicenti et al. 2005, Escaray 2007, Alonso et al. 2009a, Kristensen et al. 2014), part of Azul, Tandil, and Balcarce counties, highlights the great value of the floristic richness (mainly native and endemic taxa) of these mountains. The Tandilia system consists of mountain ranges interrupted by valleys, which extend $350 \mathrm{~km}$ from the center of Buenos Aires province to its southeastern Atlantic coast (Fig. 1). Available data from surveys, herbarium materials, and floristic inventories, include 578 species and infraspecific taxa for this area (Alonso et al. 2009b). However, this number is preliminary and could substantially increase if the mountain ranges that have not been fully inventoried were studied, such as those belonging to southeast extremity of this orographic system. The Tandilia system, near Mar del Plata, is affected by anthropogenic disturbances including farming, recreational activities, infrastructure projects, and natural resource extraction, such as mining and collection of medicinal and ornamental plants. Such alterations can cause the disappearance of native or even endemic species, their replacement by foreign floristic elements and the reduction of the ecosystem services associated with these environments (Méndez 2009, OPDS 2011, Álvarez et al. 2012, Kristensen et al. 2014, Sanhueza and Zalba 2014, Echeverría et al. 2015). Because of this, it is useful to assess the biodiversity, level of endemism, and degree of degradation of southeastern Tandilia mountain range.

Estancia Paititi is a farm in the southeastern Bue- nos Aires province that has been considered a valuable grassland area (Bilenca and Miñarro 2004) and an area of interest for conservation and ecotourism (Chebez 2005). Estancia Paititi is divided into 2 areas: 1 of them has deep fertile soils and is dedicated to organic farming (crops and pastures), while the other has 2 rocky outcrops and is a natural reserve (Paititi Natural Reserve).

The objectives of this reserve are to work actively towards the conservation and research of natural and cultural resources and to raise awareness of the care of the environment. Since 2014, Paititi Reserve is a member of the Argentine Network of Private Natural Reserves (RARNAP 2016). The reserve was divided in 2 areas, Sierra Grande, dedicated to educational, recreational, and livestock activities, and Sierra Chica, which remains untouched, with minimal anthropic interference. The latter area is set aside for research on archeology, zoology, and botany (Fig. 1). A thorough knowledge of the flora of this reserve is essential to quantify floristic richness and to develop guidelines for future studies and conservation programs. With this aim, a floristic survey of Sierra Chica, the untouched area of Paititi Natural Reserve, was done.

\section{Methods}

Study site. Paititi Natural Reserve (37 $54^{\prime} 00^{\prime \prime}$ S, $057^{\circ} 49^{\prime} 00^{\prime \prime} \mathrm{W}$; geodetic datum WGS84) is located at the southeast edge of La Peregrina mountain range, General Pueyrredón county, Buenos Aires province, Argentina. These rocky outcrops belong to the Tandilia mountain range system that traverses the province of Buenos Aires from its center to the southeast (Fig. 1). The mountains are mainly composed by orthoquartzites from the Lower Paleozoic, established over a Precambrian crystalline basement (Teruggi and Kilmurray 1980). Surrounding these mountains, the soil matrix is formed by various 
geological materials resulting from loess deposition during the late Cenozoic. The reserve is located in the Pampeana Phytogeographical Province (Pampeano Austral District), which is characterized by the presence of a climax grassland community (Bothriochloa laguroides, Nassella neesiana, Jarava plumosa, Piptochaetium montevidense and Aristida murina), bunches of Tussock Paspalum (Paspalum quadrifarium), and shrubs (Baccharis dracunculifolia ssp. tandilensis, Colletia paradoxa, and Dodonaea viscosa) (Cabrera and Zardini 1978). The climate is subhumid-humid, temperate-cold in winter and temperate-warm in summer, with a mean annual temperature of $14{ }^{\circ} \mathrm{C}$, and with maximum temperatures reaching $32{ }^{\circ} \mathrm{C}$, and minimum temperatures around $0{ }^{\circ} \mathrm{C}$. In winter, frosts occur and sometimes it snows. The annual rainfall is around $850 \mathrm{~mm}$ and although the rains are distributed throughout the year, they are most intense in winter and scarce during the summer (Falasca et al. 2000, INTA 2016). The Paititi reserve covers an area of 220 ha (RARNAP 2016) of which 40 ha are part of Sierra Chica. This is a rocky outcrop separated from Sierra Grande by a watercourse that originates in the hills of an adjoining property and whose route is accompanied by an arboreal stratum. Sierra Chica has an altitudinal range of 88 to $156 \mathrm{~m}$ above sea level. The summits and upper slopes of this mountain are variously shaded and with variable soil depth, ranging from shallow $(<10 \mathrm{~cm})$ to deep $(>60 \mathrm{~cm})$. Deep soils allow the growth of trees and shrubs (Fig. 2).

Data collection and analysis. A thorough survey of vascular plants was made from December 2013 to June 2015 in Sierra Chica. Surveys were held every $2-3$ weeks from spring to autumn, and every 4 weeks in winter, covering the periods of flowering and fructification of the different plant entities. After collecting reference plant material, the specimens were identified based on the following: Flora de la provincia de Buenos Aires (Cabrera 1963, 1965a, 1965b, 1967, 1968, 1970), Flora Rioplatense (Hurrell 2009a, 2013), and Flora Argentina (Al-Shehbaz and Salariato 2012, Múlgura et al. 2012, Zuloaga et al. 2012a, 2012b, Barboza 2013, Zuloaga et al. 2014a, 2014b). The vascular plants were classified into subclasses and superorders following Chase and Reveal (2009). Families within subclasses and superorders were according to Christenhusz et al. (2011a, 2011b) for fern and gymnosperms, respectively, and the classification system of The Angiospermae Phylogenetic Group IV (2016), for the angiosperms. Species names were updated based on the Flora Argentina database (http://www.floraargentina. edu.ar/). This database was also used to determine species' status as native (species naturally occurring in the area), adventive (exotic species, with natural introduction or mediated by humans), and cosmopolitan (species that are widely distributed). Within native category, species were classified in 3 groups according to their distribution range: species of wide distribution in the American continent, species exclusively of the Southern Cone Region of

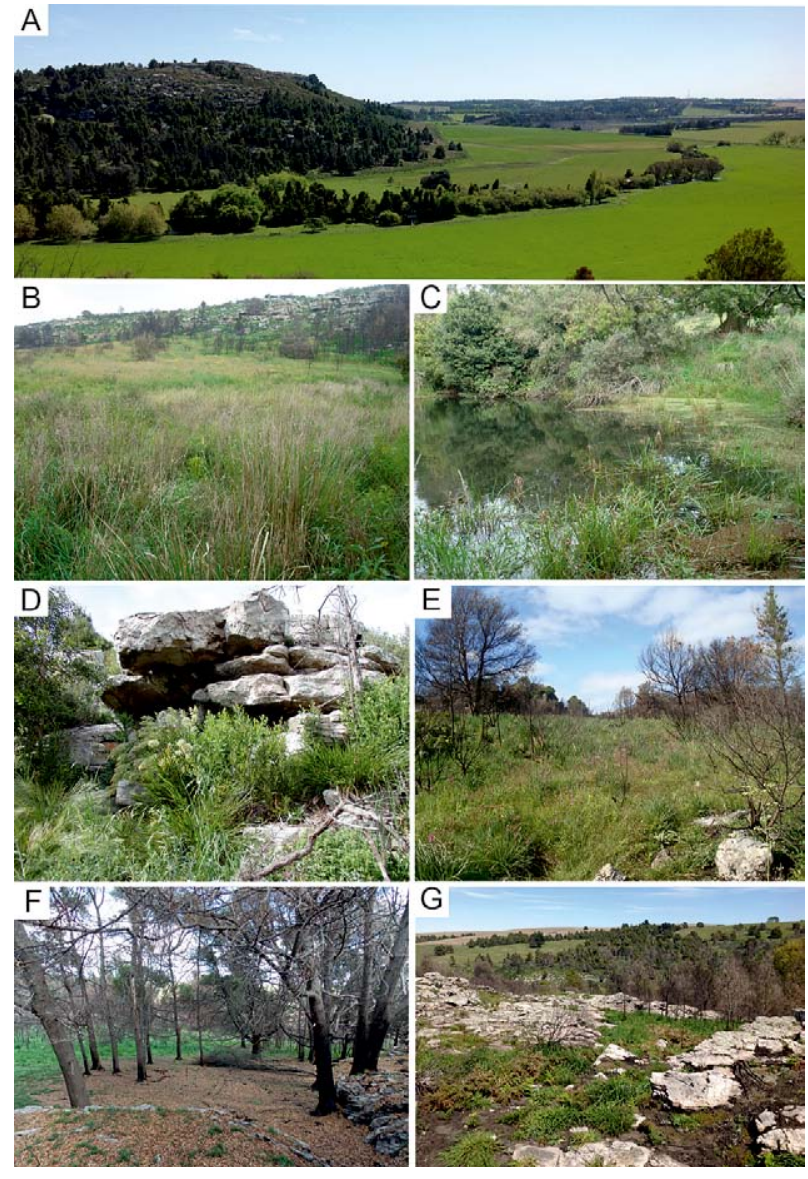

Figure 2. Study area. A. Overview of the Paititi Natural Reserve. B. Grassland area. C. Stream area. D. Mountain range slope area. E. Middle mountain range area. F. Acacias area. G. Mountain range summit area.

South America, and species endemic to mountain ranges of Buenos Aires province (Flora Argentina 2017). Species were also classified according to their type of habitat (terrestrial, aquatic floating, and aquatic marshy) and life form (therophytes, hemicryptophytes, cryptophytes, chamaephytes, lianas, epiphytes, and phanerophytes) (Raunkiaer 1934). Those species that were considered to be threatened by Delucchi (2006), according to the IUCN Red List Categories and Criteria, were noted. Reference specimens were deposited in the BAL Herbarium (E.E.A. Balcarce, Instituto Nacional de Tecnología Agropecuaria Balcarce, Buenos Aires, Argentina).

Forty-two species were described; these include 1 species with an uncertain identification, others that are newly recorded in Buenos Aires mountain ranges, and the rest that are native species (including endemic species) that, according to Delucchi (2006), are threatened. However, those newly recorded species that are frequently described in the literature as alien or native weeds, ornamental trees, fruit shrubs, forage plants, or typical members of watercourses or ponds of the province are not described.

For each of these 42 described species, their abundance was estimated at the sites where each was most frequently found: rare $=$ fewer than 5 sites; infrequent $=$ $5-9$ sites; frequent $=10-15$ sites; very frequent $\geq 16$ sites. 
Table 1. Vascular species of the untouched area of the Sierra Chica of the Paititi Natural Reserve (Tandilia mountain range system, Argentina) and synonyms more frequently used in the area. Families are sorted alphabetically within each subclass. The superorder (Chase and Reveal, 2009), is mentioned in parenthesis after the family as: AST = Asteranae; CAR = Caryophyllanae; LIL = Lilianae; MAG = Magnolianae; RAN $=$ Ranunculanae; $\mathrm{ROS}=$ Rosanae; $\mathrm{SAX}=$ Saxifraganae. This table also shows status $(\mathrm{Na}=$ native, $\mathrm{Co}=$ cosmopolitan, $\mathrm{Ad}=$ adventive), and distribution boundaries of the native species (AC = American continent, $S C=$ Southern Cone Region of South America, BA = mountain ranges of Buenos Aires province), threatened species according to Delucchi (2006) =\#, life form "LF" ( $T=$ therophytes, $C=c r y p t o p h y t e s, C h$ = chamaephytes, $\mathrm{P}=$ phanerophytes, $\mathrm{H}=$ hemicryptophytes, $\mathrm{L}=$ lianas, $\mathrm{E}=$ epyphytes), type of habitat "Hab" (Ter = terrestrial, $\mathrm{FI}=$ aquatic floating, $\mathrm{Ma}=$ aquatic marshy), location "Loc" (see Figure 3 and Table 2), references of the presence of each species in the mountain ranges of Buenos Aires province "Ref P" and references used for species identification "Ref I" (1 = Cabrera 1968, 2 = Cabrera 1970, $3=$ Cabrera 1967, 4 = Cabrera 1965a, 5 = Cabrera 1965b, 6 = Cabrera 1963, 7 = Zuloaga et al. 2012 a, $8=$ Zuloaga et al. 2012 b, $9=$ Zuloaga et al. 2014 a, $10=$ Zuloaga et al. 2014b, 11 = Múlgura et al. 2012, 12 = Barboza 2013, $13=$ Hurrell 2009a, $14=$ Grondona 1948, $15=$ Hurrell 2013, $16=$ Ariza Espinar 2005, 17 = Flora Argentina 2017, 18 = Azevedo-Gonçalves \& Matzenbacher 2006, 19 = Dimitri 1978, 20 = Al-Shehbaz \& Salariato 2012, 21 = Calviño \& Martinez 2007, 22 = Frangi 1975, 23 = Alonso et al., 2009a, 24 = Escaray 2007, 25 = Valicenti et al. 2005, $26=$ Long y Grassini 1997; 27 = Álvarez et al. 2012, 28 = Valicenti et al. 2000, 29 = Frangi \& Bottino 1995), voucher collectors "Voucher" (A = S.I. Alonso, C = V.M. Comparatore, $\mathrm{E}=$ M.L. Echeverría, $\mathrm{CL}=\mathrm{A} . \mathrm{M}$. Clausen, $\mathrm{NU}=\mathrm{M}$.C. Nuciari, $\mathrm{LM}=\mathrm{L}$. Montes, $\mathrm{V}=\mathrm{C} . \mathrm{B}$. Villamil, $\mathrm{O}=\mathrm{K} . \mathrm{A}$. Okada, $\mathrm{FCA} s / \mathrm{n}=$ without number, donated by the Identification Species Service of the FCA, UNMdP) and the BAL Herbarium number "BAL" within parenthesis. The "ACE" (Alonso S.I., Comparatore V.M. and Echeverría M.L.) and "E" (Echeverría M.L.) collections belong to the Paititi Reserve.

\begin{tabular}{|c|c|c|c|c|c|c|c|}
\hline Taxon & Status, \# & LF & Hab & Loc & Ref P & Ref I & Voucher and BAL \\
\hline \multicolumn{8}{|l|}{ Subclass Polypodiae } \\
\hline \multicolumn{8}{|l|}{ Azollaceae } \\
\hline Azolla filiculoides Lam. & $\mathrm{Na}, \mathrm{AC}$ & $\mathrm{T}$ & $\mathrm{FI}$ & 1 & 28 & 1,17 & ACE 3990 (8221) \\
\hline \multicolumn{8}{|l|}{ Blechnaceae } \\
\hline $\begin{array}{l}\text { Blechnum australe L. ssp. auriculatum (Cav.) de la Sota (=B. } \\
\text { auriculatum Cav.) }\end{array}$ & $\mathrm{Na}, \mathrm{SC}$ & $C$ & Ter & 18 & 1 & 1,17 & ACE 4011 (8222), A 844 (109) \\
\hline \multicolumn{8}{|l|}{ Dennstaedtiaceae } \\
\hline $\begin{array}{l}\text { Pteridium arachnoideum (Kaulf.) Maxon (=P. aquilinum (L.) Kuhn } \\
\text { var. arachnoideum (Kaulf.) Herter) }\end{array}$ & $\mathrm{Na}, \mathrm{AC}$ & $C$ & Ter & 21 & 1 & 1,17 & ACE 3646 (8223), A 842 (295) \\
\hline \multicolumn{8}{|l|}{ Dryopteridacea } \\
\hline Polystichum montevidense (Spreng.) Rosenst. & $\mathrm{Na}, \mathrm{AC}$ & $\mathrm{C}$ & Ter & 15 & 1 & 1,17 & O 6608 (4054), FCA s/n (6173) \\
\hline Rumohra adiantiformis (G. Forst.) Ching & $\mathrm{Na}, \mathrm{AC}$ & $\mathrm{C}$ & Ter & 15 & 22 & 1,17 & ACE 4114 (8830), O 3132 (4062) \\
\hline \multicolumn{8}{|l|}{ Pteridaceae } \\
\hline Adiantum raddianum C. Presl & $\mathrm{Na}, \mathrm{AC} / \#$ & $\mathrm{C}$ & Ter & 3 & 1 & 1,17 & ACE 4012 (8224), FCA s/n (7273) \\
\hline $\begin{array}{l}\text { Cassebeera triphylla (Lam.) Kaulf. (=Doryopteris tryphylla (Lam.) } \\
\text { Christ. }\end{array}$ & $\mathrm{Na}, \mathrm{AC}$ & $C$ & Ter & 13 & 1 & 1,17 & ACE 4085 (8829), O 1627 (4056) \\
\hline Pellaea ternifolia (Cav.) Link & $\mathrm{Na}, \mathrm{AC}$ & $\mathrm{C}$ & Ter & 20 & 1 & 1,17 & ACE 3490 (8225), O 6607 (4052) \\
\hline \multicolumn{8}{|l|}{ Woodsiaceae } \\
\hline Woodsia montevidensis (Spreng.) Hieron. & $\mathrm{Na}, \mathrm{AC}$ & $\mathrm{C}$ & Ter & 11 & 1 & 1,17 & ACE 3651 (8226), A 855 (108) \\
\hline \multicolumn{8}{|l|}{ Subclass Pinidae } \\
\hline \multicolumn{8}{|l|}{ Pinaceae } \\
\hline Pinus radiata $\mathrm{D}$. Don & Ad & $\mathrm{P}$ & Ter & 20 & 26 & 19 & ACE 4027 (8227), FCA s/n (6260) \\
\hline Pinus sylvestris L. & $\mathrm{Ad}$ & $\mathrm{P}$ & Ter & 10 & No & 19 & ACE 3935 (8228) \\
\hline \multicolumn{8}{|l|}{ Subclass Magnoliidae (Dicotyledon species) } \\
\hline \multicolumn{8}{|l|}{ Acanthaceae (AST) } \\
\hline Stenandrium dulce (Cav.) Nees (=S. trinerve Nees) & $\mathrm{Na}, \mathrm{AC}$ & $\mathrm{H}$ & Ter & 13 & 26 & 5,17 & ACE 3752 (8229), NU 403 (2280) \\
\hline \multicolumn{8}{|l|}{ Adoxaceae (AST) } \\
\hline Sambucus australis Cham. \& Schltdl. & $\mathrm{Na}, \mathrm{AC}$ & $\mathrm{P}$ & Ter & 3 & No & 5,17 & ACE 4115 (8822), Alo 1186 (627) \\
\hline \multicolumn{8}{|l|}{ Amaranthaceae (CAR) } \\
\hline Alternanthera philoxeroides (Mart.) Griseb. f. philoxeroides & $\mathrm{Na}, \mathrm{AC}$ & $\mathrm{H}$ & $\mathrm{FI}$ & 1 & No & 3,17 & ACE 4000 (8230), A 761 (285) \\
\hline Amaranthus hybridus L. ssp. hybridus (=A. quitensis Kunth) & Ad & $\mathrm{T}$ & Ter & 3 & No & 3,17 & ACE 3593 (8231), O 1555 (4697) \\
\hline Gomphrena perennis L. var. perennis & $\mathrm{Na}, \mathrm{AC}$ & $\mathrm{H}$ & Ter & 13 & 22 & 3,17 & ACE 3491 (8233), LM 696 (724) \\
\hline Pfaffia gnaphalioides (L. f.) Martius & $\mathrm{Na}, \mathrm{AC}$ & $\mathrm{H}$ & Ter & 13 & 3 & 3,17 & ACE 3669 (8232), NU 818 (3055) \\
\hline \multicolumn{8}{|l|}{ Apiaceae (AST) } \\
\hline Ammi majus L. & Ad & $\mathrm{T}$ & Ter & 7 & 23 & 4,15 & ACE 3671 (8234), O 5313 (4111) \\
\hline Bowlesia incana Ruiz \& Pav. & $\mathrm{Na}, \mathrm{AC}$ & $\mathrm{T}$ & Ter & 7 & 26 & 4,17 & O 4113 (4083), FCA s/n (7068) \\
\hline Conium maculatum L. & Ad & $\mathrm{T}$ & Ter & 8 & 25 & 4,17 & ACE 3680 (8235), O 3317 (4093) \\
\hline $\begin{array}{l}\text { Cyclospermum leptophyllum (Pers.) Sprague (=Apium } \\
\text { leptophyllum (Pers.) Muell.) }\end{array}$ & $\mathrm{Na}, \mathrm{AC}$ & $\mathrm{T}$ & Ter & 8 & 23 & 4,17 & ACE 4075 (8823), O 2442 (4096) \\
\hline Eryngium elegans Cham. \& Schltdl. & $\mathrm{Na}, \mathrm{AC}$ & $\mathrm{H}$ & Ter & 14 & 22 & 4,17 & ACE 3885 (8236), NU 205 (6111) \\
\hline Eryngium horridum Malme & $\mathrm{Na}, \mathrm{AC}$ & $\mathrm{H}$ & Ter & 11 & 22 & 4,17 & ACE 3624 (8237), O 1461 (4066) \\
\hline Eryngium nudicaule Lam. & $\mathrm{Na}, \mathrm{AC}$ & $\mathrm{H}$ & Ter & 18 & 22 & 4,17 & ACE 3664 (8238), O 1696 (4069) \\
\hline Eryngium regnellii Malme & $\mathrm{Na}, \mathrm{AC}$ & $\mathrm{H}$ & Ter & 11 & 21 & 17,21 & ACE 4031 (8239) \\
\hline Eryngium serra Cham. \& Schltdl. & $\mathrm{Na}, \mathrm{SC} / \#$ & $\mathrm{H}$ & Ter & 14 & 22 & 4,17 & ACE $3611 \mathrm{~b}(8240)$, O 2319 (4078) \\
\hline Foeniculum vulgare Mill. & Ad & $\mathrm{H}$ & Ter & 11 & 24 & 4,17 & ACE 3673 (8241), O 1700 (4084) \\
\hline Hydrocotyle bonariensis Lam. & $\mathrm{Na}, \mathrm{AC}$ & $\mathrm{H}$ & $\mathrm{Ma}$ & 4 & 22 & 4,17 & ACE 4103 (8824), LM 431 (767) \\
\hline \multicolumn{8}{|l|}{ Apocynaceae (AST) } \\
\hline Araujia sericifera Brot. (=A. hortorun Fournier) & $\mathrm{Na}, \mathrm{AC}$ & $\mathrm{L}$ & Ter & 7 & 23 & 5,17 & ACE 3953a (8242) \\
\hline Oxypetalum solanoides Hook. \& Arn. & $\mathrm{Na}, \mathrm{AC}$ & $\mathrm{Ch}$ & Ter & 11 & 23 & 5,17 & ACE 3848 (8243), NU 694 (2853) \\
\hline
\end{tabular}


Table 1. Continued.

\begin{tabular}{|c|c|c|c|c|c|c|c|}
\hline Taxon & Status, \# & LF & Hab & Loc & Ref $P$ & Ref I & Voucher and BAL \\
\hline \multicolumn{8}{|l|}{ Araliaceae (AST) } \\
\hline Hedera helix $\mathrm{L}$. & $\mathrm{Ad}$ & $\mathrm{L}$ & Ter & 2 & 26 & 4,17 & ACE 3687 (8244), FCA s/n (6031) \\
\hline \multicolumn{8}{|l|}{ Asteraceae (AST) } \\
\hline $\begin{array}{l}\text { Acanthostyles buniifolius (Hook. \& Arn.) R. M. King \& H. Rob. } \\
\text { (=Eupatorium buniifolius Hooker et Arnot) }\end{array}$ & $\mathrm{Na}, \mathrm{AC} / \#$ & $P$ & Ter & 11 & 6 & $6 ; 9,17$ & ACE 4021 (8245), A 775 (292) \\
\hline Achyrocline satureioides (Lam.) DC. & $\mathrm{Na}, \mathrm{AC}$ & $\mathrm{Ch}$ & Ter & 17 & 6 & $6,9,17$ & ACE 4074 (8825), V 4021 (2441) \\
\hline Ambrosia tenuifolia Spreng. & $\mathrm{Na}, \mathrm{AC}$ & $\mathrm{Ch}$ & Ter & 8 & 23 & $6,9,17$ & ACE 3608 (8246), LM 707 (695) \\
\hline Arctium minus (Hill) Bernh. & $\mathrm{Ad}$ & $\mathrm{T}$ & Ter & 3 & No & $6,9,17$ & ACE 4001 (8247), A 704 (327) \\
\hline Baccharis articulata (Lam) Pers. & $\mathrm{Na}, \mathrm{AC}$ & $\mathrm{P}$ & Ter & 9 & 22 & $6,9,16,17$ & ACE 4077 (8248), O 3205 (3296) \\
\hline Baccharis crispa Spreng. & $\mathrm{Na}, \mathrm{AC}$ & $\mathrm{Ch}$ & Ter & 19 & 6 & $6,9,16,17$ & ACE 3628 (8518), O 2515 (3293) \\
\hline Baccharias coridifolia DC. & $\mathrm{Na}, \mathrm{AC}$ & $\mathrm{P}$ & Ter & 11 & 22 & $6,9,16,17$ & ACE 4034 (8249), A 868 (356) \\
\hline $\begin{array}{l}\text { Baccharis dracunculifolia DC. ssp. tandilensis (Speg.) Giuliano } \\
\text { (=B. tandilensis Speg.) }\end{array}$ & $\mathrm{Na}, \mathrm{BA} / \#$ & $\mathrm{P}$ & Ter & 11 & 6 & $6,9,17$ & ACE 4078 (8250), O 2547 (3312) \\
\hline Baccharis glutinosa Pers. (=B. pingrae DC var. pingrae) & $\mathrm{Na}, \mathrm{AC}$ & $\mathrm{C}$ & Ter & 4 & 23 & $6,9,16,17$ & ACE 4072 (8812), V 3802 (2448) \\
\hline $\begin{array}{l}\text { Baccharis linearifolia (Lam.) Pers. ssp. linearifolia (=B. rufescens } \\
\text { Sprengel var. ventanicola Cabrera) }\end{array}$ & $\mathrm{Na}, \mathrm{AC}$ & $\mathrm{Ch}$ & Ter & 9 & 6 & $6,9,17$ & ACE 4080 (8813), O 1678 (3305) \\
\hline Berroa gnaphalioides (Less.) Beauverd & $\mathrm{Na}, \mathrm{AC}$ & $\mathrm{H}$ & Ter & 13 & No & $6,9,15,17$ & ACE 3879 (8251), O 1915 (3285) \\
\hline Bidens subalternans DC. var. subalternans & $\mathrm{Na}, \mathrm{AC}$ & $\mathrm{T}$ & Ter & 3 & No & 6,17 & ACE 3691 (8252), LM 968 (2233) \\
\hline $\begin{array}{l}\text { Campovassouria cruciata (Vell.) R. M. King \& H. Rob. } \\
\text { (=Eupatorium bupleurifolium DC) }\end{array}$ & $\mathrm{Na}, \mathrm{AC}$ & $P$ & Ter & 16 & 6 & $6,9,17$ & ACE 4053 (8253), LM 705 (722), \\
\hline $\begin{array}{l}\text { Campuloclinium macrocephalum (Less.) D. C. (=Eupatorium } \\
\text { macrocephalum Less.) }\end{array}$ & $\mathrm{Na}, \mathrm{AC}$ & $\mathrm{H}$ & Ter & 14 & 6 & $6,9,17$ & E 143 (8254), CL 929 (2105) \\
\hline Carduus acanthoides $\mathrm{L}$. & $\mathrm{Ad}$ & $\mathrm{T}$ & Ter & 20 & 23 & $6,9,17$ & ACE 4083 (8837), A 609 (186) \\
\hline Carduus pycnocephalus L. & $\mathrm{Ad}$ & $\mathrm{T}$ & Ter & 3 & 23 & $6,9,17$ & ACE 4055 (8255), NU 802 (2999) \\
\hline Carthamus lanatus L. & $\mathrm{Ad}$ & $\mathrm{T}$ & Ter & 7 & No & $6,9,17$ & ACE 4084 (8826), A 835 (293) \\
\hline Centaurea calcitrapa L. & $\mathrm{Ad}$ & $\mathrm{T}$ & Ter & 7 & 22 & $6,9,17$ & ACE 4087 (8827), A 1079 (528) \\
\hline Chaptalia exscapa (Pers.) Baker var. exscapa & $\mathrm{Na}, \mathrm{AC}$ & $\mathrm{H}$ & Ter & 13 & 26 & 6,17 & A 1173 (5454), A 1173 (825) \\
\hline Chaptalia piloselloides (Vahl) Baker & $\mathrm{Na}, \mathrm{SC}$ & $\mathrm{H}$ & Ter & 13 & 22 & 6,17 & ACE 4037 (8256) \\
\hline $\begin{array}{l}\text { Chromolaena hirsuta (Hook. \& Arn.) R. M. King \& H. Rob. } \\
\text { (=Eupatorium subhastatum Hooker \& Arnott) }\end{array}$ & $\mathrm{Na}, \mathrm{SC}$ & $\mathrm{H}$ & Ter & 14 & 6 & $6,9,17$ & ACE 3465 (8257), A 872 (741) \\
\hline $\begin{array}{l}\text { Chromolaena squarrulosa (Hook. \& Arn.) R. M. King \& H. Rob. } \\
\text { (=Eupatorium squarrulosum Hooker et Arnott) }\end{array}$ & $\mathrm{Na}, \mathrm{SC} / \#$ & $\mathrm{H}$ & Ter & 14 & 6 & $6,9,17$ & E 187 (8258), A 875 (755) \\
\hline Chrysolaena flexuosa (Sims) H. Rob. (=Vernonia flexuosa Sims) & $\mathrm{Na}, \mathrm{AC}$ & $\mathrm{H}$ & Ter & 14 & 22 & $6,10,17$ & ACE 4150 (8259), A 829 (890) \\
\hline Cirsium vulgare (Savi) Ten. & $\mathrm{Ad}$ & $\mathrm{T}$ & Ter & 12 & 23 & $6,9,17$ & ACE 4088 (8826), A 516 (188) \\
\hline $\begin{array}{l}\text { Conyza bonariensis (L.) Cronquist var. angustifolia (Cabrera) } \\
\text { Cabrera }\end{array}$ & $\mathrm{Na}, \mathrm{AC}$ & $\mathrm{T}$ & Ter & 19 & No & $6,9,16,17$ & ACE $4040 \mathrm{~b}(8260)$ \\
\hline Conyza bonariensis (L.) Cronquist var. bonariensis Colla & $\mathrm{Na}, \mathrm{AC}$ & $\mathrm{T}$ & Ter & 11 & 24 & $6,9,16,17$ & 4040a (8261), O 1663 (3053) \\
\hline $\begin{array}{l}\text { Conyza sumatrensis (Retz.) E. Walker var. leiotheca (S. F. Blake) } \\
\text { Pruski \& G. Sancho (=C. floribunda H.B.K.) }\end{array}$ & $\mathrm{Na}, \mathrm{AC}$ & $\mathrm{T}$ & Ter & 11 & 24 & $6,9,16,17$ & ACE 4039 (8262) \\
\hline $\begin{array}{l}\text { Conyza sumatrensis (Retz.) E. Walker var. sumatrensis (=Conyza } \\
\text { bonariensis (L.) Cronquist var. microcephala (Cabrera) Cabrera) }\end{array}$ & $\mathrm{Na}, \mathrm{AC}$ & $\mathrm{T}$ & Ter & 19 & No & $6,9,16,17$ & ACE 4058 (8263) \\
\hline Cotula australis (Sieber ex Spreng.) Hook. F. & $\mathrm{Ad}$ & $\mathrm{T}$ & Ter & 3 & No & $6,9,15,17$ & O 6931 (4732), O 6931 (4732) \\
\hline Crepis capillaris (L.) Wallr. & $\mathrm{Ad}$ & $\mathrm{T}$ & Ter & 17 & 24 & 6,17 & ACE 4056 (8264), O 1471 (3072) \\
\hline Criscia stricta (Spreng.) Katinas (=Trixis stricta Less.) & $\mathrm{Na}, \mathrm{AC}$ & $\mathrm{H}$ & Ter & 13 & 6 & 6,17 & ACE 4090 (8836), O 2256 (3121) \\
\hline Cynara cardunculus $\mathrm{L}$. & $\mathrm{Ad}$ & $\mathrm{H}$ & Ter & 18 & 23 & $6,9,17$ & ACE 4091 (8835), FCA s/n (5455) \\
\hline Facelis retusa (Lam.) Sch. Bip. & $\mathrm{Na}, \mathrm{AC} / \#$ & $\mathrm{~T}$ & Ter & 13 & 22 & $6,9,15,17$ & O 4705 (3099), O 4705 (3099) \\
\hline Galinsoga parviflora Cav. & $\mathrm{Na}, \mathrm{AC}$ & $\mathrm{T}$ & Ter & 3 & No & 6,17 & ACE 3666 (8265), FCA s/n (5490) \\
\hline Gamochaeta americana (Mill.) Wedd & $\mathrm{Na}, \mathrm{AC}$ & $\mathrm{T}$ & Ter & 18 & 15 & $6,9,15,17$ & ACE $3900(8266)$ \\
\hline Gamochaeta argentina Cabrera & $\mathrm{Na}, \mathrm{SC}$ & $\mathrm{T}$ & Ter & 15 & 6 & $6,9,15,17$ & ACE 3186 (8267), A 1445 (2306) \\
\hline Gamochaeta coarctata (Willd.) Kerguélen (=G. spicata Cabrera) & $\mathrm{Na}, \mathrm{AC}$ & $\mathrm{H}$ & Ter & 13 & 23 & $6,9,15,17$ & ACE 3552 (8268), O 1867 (3268) \\
\hline Gamochaeta falcata (Lam.) Cabrera & $\mathrm{Na}, \mathrm{AC}$ & $\mathrm{H}$ & Ter & 11 & No & $6,9,15,17$ & ACE 3616 (8269) \\
\hline Gamochaeta filaginea (D.C.) Cabrera & $\mathrm{Na}, \mathrm{SC}$ & $\mathrm{H}$ & Ter & 18 & 22 & $6,9,15,17$ & ACE 3938 (8270), O 2624 (3276) \\
\hline $\begin{array}{l}\text { Gamochaeta pensylvanica (Willd.) Cabrera (=G. platensis } \\
\text { (Cabrera) Cabrera) }\end{array}$ & $\mathrm{Na}, \mathrm{AC} / \#$ & $\mathrm{H}$ & Ter & 21 & No & $6,9,15,17$ & ACE 3182 (8271), O 2258 (3274) \\
\hline Gamochaeta simplicicaulis (Willd. ex Spreng.) Cabrera & $\mathrm{Na}, \mathrm{AC}$ & $\mathrm{T}$ & Ter & 13 & No & $6,9,15,17$ & ACE 3901 (8272), FCA s/n (5497) \\
\hline Gamochaeta stachydifolia (Lam.) Cabrera & $\mathrm{Na}, \mathrm{AC}$ & $\mathrm{H}$ & Ter & 15 & 6 & $6,9,15,17$ & ACE 3184 (8273), CL 194 (2329) \\
\hline $\begin{array}{l}\text { Gyptis tanacetifolia (Gillies ex Hook. \& Arn.) D. J. N. Hind \& Flann } \\
\text { (=Eupatorium tanacetifolium Gill.) }\end{array}$ & $\mathrm{Na}, \mathrm{SC}$ & $\mathrm{H}$ & Ter & 13 & 6 & $6,9,17$ & ACE 4101 (8831), A 739 (333) \\
\hline Helianthus annuus L. & $\mathrm{Ad}$ & $\mathrm{T}$ & Ter & 4 & No & 6,17 & A 663 (383), A 663 (383) \\
\hline Helminthotheca echioides (L.) Holub. (=Picris echioides L.) & $\mathrm{Ad}$ & $\mathrm{T}$ & Ter & 7 & No & $6,15,17$ & ACE 4109 (8832), O 1524 (3169) \\
\hline Hieracium tandilense Sleumer & $\mathrm{Na}, \mathrm{BA} / \#$ & $\mathrm{C}$ & Ter & 15 & 6 & $6,15,17$ & ACE 3933a (8274), NU 538 (2120) \\
\hline Hypochaeris chillensis (Kunth) Hieron. (=H. tweediei Cabr.) & $\mathrm{Na}, \mathrm{AC}$ & $\mathrm{H}$ & Ter & 11 & 24 & $6,15,17$ & ACE 3983 (8275), O 2272 (3359) \\
\hline $\begin{array}{l}\text { Hypochaeris neopinnatifida Azevédo-Gonçalves \& Matzenb. } \\
\text { (=H. rosengurttii var. pinnatifida Cabr.) }\end{array}$ & $\mathrm{Na}, \mathrm{SC} / \#$ & $\mathrm{H}$ & Ter & 10 & 6 & $6,17,18$ & ACE 3764 (8276), O 4659 (3347) \\
\hline Hypochaeris pampasica Cabrera & $\mathrm{Na}, \mathrm{SC} / \#$ & $\mathrm{H}$ & Ter & 11 & 24 & $6,15,17$ & ACE 3852 (8277),NU 502 (5524) \\
\hline Hypochaeris radicata $\mathrm{L}$. & $\mathrm{Ad}$ & $\mathrm{H}$ & Ter & 11 & 22 & $6,15,17$ & ACE 3980 (8278), A 592 (386) \\
\hline Hysterionica pinifolia (Poir.) Baker & $\mathrm{Na}, \mathrm{AC}$ & $\mathrm{C}$ & Ter & 13 & 6 & $6,9,17$ & E 146 (8279), LM 697 (719) \\
\hline
\end{tabular}


Table 1. Continued.

\begin{tabular}{|c|c|c|c|c|c|c|c|}
\hline Taxon & Status, \# & LF & Hab & Loc & Ref $\mathbf{P}$ & Ref I & Voucher and BAL \\
\hline Lactuca serriola $\mathrm{L}$. & Ad & $\mathrm{T}$ & Ter & 18 & 23 & $6,9,17$ & ACE 3187 (8280), A 821 (547) \\
\hline Lucilia acutifolia (Poir.) Cass. & $\mathrm{Na}, \mathrm{AC}$ & $\mathrm{H}$ & Ter & 13 & 6 & $6,9,15,17$ & E 136 (8281), NU 812 (3009) \\
\hline Micropsis sphatulata (Pers.) Cabrera & $\mathrm{Na}, \mathrm{AC}$ & $\mathrm{T}$ & Ter & 18 & No & $6,9,15,17$ & ACE 3906 (8282), A 1447 (2308) \\
\hline Panphalea heterophylla Less. & $\mathrm{Na}, \mathrm{AC}$ & $\mathrm{T}$ & Ter & 18 & 6 & 6,17 & O 3227 (3166), FCA s/n (5550) \\
\hline Podocoma hirsuta (Hook. \& Arn.) Baker & $\mathrm{Na}, \mathrm{AC}$ & $\mathrm{C}$ & Ter & 8 & 6 & $6,9,16,17$ & ACE 3467 (8283), CL 1119 (6830) \\
\hline $\begin{array}{l}\text { Pseudognaphalium gaudichaudianum (DC.) Anderb. } \\
\text { (=Gnaphalium gaudichaudianum DC) }\end{array}$ & $\mathrm{Na}, \mathrm{AC}$ & $\mathrm{T}$ & Ter & 15 & 24 & $6,9,15,17$ & ACE 3936a (8284), LM 955 (2720) \\
\hline Pterocaulon cordobense Kuntze & $\mathrm{Na}, \mathrm{AC}$ & $\mathrm{H}$ & Ter & 11 & No & $6,15,17$ & E 139 (8365) \\
\hline Senecio grisebacchii Baker var. subincanus Cabrera & $\mathrm{Na}, \mathrm{SC}$ & $\mathrm{Ch}$ & Ter & 20 & 22 & $\begin{array}{l}6,10,15 \\
17\end{array}$ & ACE 3893 (8285), A 1226 (5591) \\
\hline Senecio madagascariensis Poir. (=S. burchelli DC) & $\mathrm{Ad}$ & $\mathrm{Ch}$ & Ter & 18 & 23 & $\begin{array}{c}6,10,15 \\
17\end{array}$ & ACE 4116 (8833), LM 649 (5592) \\
\hline Senecio selloi (Spreng.) DC. & $\mathrm{Na}, \mathrm{SC} / \#$ & $\mathrm{Ch}$ & Ter & 21 & 22 & $\begin{array}{l}6,10,15 \\
17\end{array}$ & ACE 4117 (8834), V 3576 (2540) \\
\hline Senecio vulgaris $\mathrm{L}$. & $\mathrm{Ad}$ & $\mathrm{T}$ & Ter & 13 & No & $\begin{array}{l}6,10,15 \\
17\end{array}$ & ACE 3642 (8286), NU 48 (366) \\
\hline Silybum marianum (L.) Gaertn & Ad & $\mathrm{T}$ & Ter & 19 & 23 & $6,9,15,17$ & O 4167 (3128), FCA s/n (5604) \\
\hline Solidago chilensis Meyen & $\mathrm{Na}, \mathrm{AC}$ & $\mathrm{C}$ & Ter & 8 & 23 & $6,9,16,17$ & ACE 4120 (8835), NU 379 (5605) \\
\hline Soliva sessilis Riuz \& Pav. (=S. pterosperma Less.) & $\mathrm{Na}, \mathrm{AC}$ & $\mathrm{T}$ & Ter & 13 & 22 & $6,9,15,17$ & ACE 3805 (8287), O 3204 (3239) \\
\hline Sommerfeltia spinulosa (Spreng.) Less. & $\mathrm{Na}, \mathrm{SC} / \#$ & $\mathrm{Ch}$ & Ter & 18 & 6 & $6,9,17$ & ACE 3486 (8288), O 3138 (3250) \\
\hline Sonchus asper (L.) Hill & Ad & $\mathrm{T}$ & Ter & 18 & 25 & $6,9,15,17$ & O 3134 (4098), ACE 4148 (8289) \\
\hline Sonchus oleraceus L. & Ad & $\mathrm{T}$ & Ter & 18 & 24 & $6,9,15,17$ & ACE 4121 (8806), A 1243 (756) \\
\hline $\begin{array}{l}\text { Stevia satureiifolia (Lam.) Sch. Bip. ex Klotzsch var. patagonica } \\
\text { Hieron. }\end{array}$ & $\mathrm{Na}, \mathrm{SC} / \#$ & $\mathrm{Ch}$ & Ter & 13 & 6 & $6,9,17$ & E 147 (8290), CL 1055 (2810) \\
\hline $\begin{array}{l}\text { Symphyotrichum squamatum (Spreng.) G. L. Nesom (=Aster } \\
\text { squamatus Hieron.) }\end{array}$ & $\mathrm{Na}, \mathrm{AC}$ & $\mathrm{C}$ & Ter & 10 & 23 & $6,9,16,17$ & ACE 4076 (8836), NU 496 (5381) \\
\hline Tagetes minuta L. & $\mathrm{Na}, \mathrm{AC}$ & $\mathrm{T}$ & Ter & 7 & 26 & 6,17 & ACE 4122 (8291), O 1557 (3127) \\
\hline Taraxacum officinale F. H. Wigg. & Ad & $\mathrm{H}$ & Ter & 3 & 24 & $6,9,17$ & ACE 4123 (8840), O 4092 (3123) \\
\hline Xanthium spinosum L. & $\mathrm{Na}, \mathrm{AC}$ & $\mathrm{T}$ & Ter & 7 & 26 & 6,17 & ACE 4126 (8838), O 1559 (3316) \\
\hline \multicolumn{8}{|l|}{ Boraginaceae (AST) } \\
\hline Echium plantagineum $\mathrm{L}$. & Ad & $\mathrm{T}$ & Ter & 8 & 23 & 5,17 & E 138 (8292), O 1556 (4429) \\
\hline \multicolumn{8}{|l|}{ Brassicaceae (ROS) } \\
\hline Brassica rapa L. (= B. campestris L.) & Ad & $\mathrm{T}$ & Ter & 7 & No & 3,20 & ACE 4081 (8839), A 582 (57) \\
\hline Cardamine chenopodiifolia Pers. & $\mathrm{Na}, \mathrm{AC}$ & $\mathrm{T}$ & Ma & 4 & 3 & 3,20 & ACE 3607 (8293), LM 836 (750) \\
\hline Hirschfeldia incana (L.) Lagr.-Foss. & Ad & $\mathrm{T}$ & Ter & 7 & 26 & 3,20 & ACE 3698 (8294), O 4164 (3382) \\
\hline Lepidium bonariense $\mathrm{L}$. & $\mathrm{Na}, \mathrm{AC}$ & $\mathrm{T}$ & Ter & 11 & 23 & 3,20 & ACE 3810 (8295), CL 279 (5977) \\
\hline Lepidium didymum L. (=Coronopus didymus (L.) Smith) & $\mathrm{Na}, \mathrm{AC}$ & $\mathrm{T}$ & Ter & 13 & 26 & 3,20 & ACE 3602 (8296), O 4118 (3432) \\
\hline Lepidium tandilense Boelcke & $\mathrm{Na}, \mathrm{SC} / \#$ & $\mathrm{~T}$ & Ter & 11 & 24 & 3,20 & ACE 3641a (8297), FCA s/n (5983) \\
\hline $\begin{array}{l}\text { Nasturtium officinale W. T. Aiton (=Rorippa nasturtium- } \\
\text { aquaticum (L.) Hayek) }\end{array}$ & Ad & $\mathrm{H}$ & Ma & 14 & 22 & 3,20 & O 4191 (3408), CL 41 (5986) \\
\hline Raphanus sativus L. & Ad & $\mathrm{T}$ & Ter & 17 & 25 & 3,20 & O 4158 (3368), CL 49 (5988) \\
\hline Rapistrum rugosum (L.) All. ssp. rugosum & Ad & $\mathrm{T}$ & Ter & 3 & No & 3,20 & ACE 3670 (8298), O 1531 (3409) \\
\hline Sisymbrium officinale (L.) Scop. & Ad & $\mathrm{T}$ & Ter & 3 & 26 & 3,20 & ACE 4118 (8841), A 1449 (2302) \\
\hline \multicolumn{8}{|l|}{ Cactaceae (CAR) } \\
\hline Gymnocalycium gibbosum (Haw.) Pfeiff. ex Mittler & $\mathrm{Na}, \mathrm{SC} / \#$ & $\mathrm{H}$ & Ter & 20 & 4 & 4,17 & ACE 4100 (8810) \\
\hline \multicolumn{8}{|l|}{ Campanulaceae (AST) } \\
\hline $\begin{array}{l}\text { Triodanis perfoliata (L.) Nieuwl. ssp. biflora (Ruiz \& Pav.) } \\
\text { Lammers (=T. biflora (Ruiz \& Pavon) Greene) }\end{array}$ & $\mathrm{Na}, \mathrm{AC}$ & $\mathrm{T}$ & Ter & 8 & 24 & 5,17 & O 5191 (4466), O 4742 (4465) \\
\hline Wahlenbergia linarioides (Lam.) A. DC. & $\mathrm{Na}, \mathrm{AC}$ & $\mathrm{H}$ & Ter & 13 & 5 & 5,17 & ACE 3858 (8299), Nu 326 (2278) \\
\hline \multicolumn{8}{|l|}{ Caryophyllaceae (CAR) } \\
\hline Cerastium glomeratum Thuill. & Ad & $\mathrm{T}$ & Ter & 11 & 22 & 3,17 & ACE 3170 (8300), Alo 1473 (7076) \\
\hline Cerastium mollissimum Phil. var. mollissimum & $\mathrm{Na}, \mathrm{AC}$ & $\mathrm{H}$ & Ter & 11 & 3 & 3,17 & ACE 3783 (8301), O 4136 (4262) \\
\hline Paronychia brasiliana DC. & $\mathrm{Na}, \mathrm{AC}$ & $\mathrm{H}$ & Ter & 11 & 26 & 3,17 & ACE 3770 (8302), Alo 1430 (2200) \\
\hline Silene antirrhina L. var. antirrhina & Ad & $\mathrm{T}$ & Ter & 11 & 3 & 3,17 & ACE 3781 (8303), O 2260 (4267) \\
\hline Silene gallica $\mathrm{L}$. & $\mathrm{Ad}$ & $\mathrm{T}$ & Ter & 11 & 22 & 3,17 & ACE 3668 (8304), O 1692 (4270) \\
\hline Spergula grandis Pers. (=Spergularia grandis (Pers.) Saint Hilaire) & $\mathrm{Na}, \mathrm{SC}$ & $\mathrm{H}$ & Ter & 11 & 26 & 3,17 & ACE 3647 (8305) \\
\hline $\begin{array}{l}\text { Spergula ramosa (Cambess.) D. Dietr. var. ramosa (=Spergularia } \\
\text { ramosa Cambess.) }\end{array}$ & $\mathrm{Na}, \mathrm{AC}$ & $\mathrm{H}$ & Ter & 11 & 23 & 3,17 & ACE 3707 (8306), Nu 5 (683) \\
\hline Spergula villosa Pers. (=Spergularia villosa (Pers.) Cambess.) & $\mathrm{Na}, \mathrm{AC}$ & $\mathrm{H}$ & Ter & 13 & No & 3,17 & ACE 3639 (8307), FCA s/n (7082) \\
\hline Stellaria media (L.) Cirillo & Ad & $\mathrm{T}$ & Ter & 7 & 26 & 3,17 & ACE 3601b (8308), O $4123(4260)$ \\
\hline \multicolumn{8}{|l|}{ Celtidaceae (ROS) } \\
\hline Celtis australis L. & Ad & $\mathrm{P}$ & Ter & 3 & No & 19 & ACE 4003 (8309), FCA s/n (5836) \\
\hline $\begin{array}{l}\text { Celtis ehrenbergiana (Klotzsch) Liebm. var. ehrenbergiana } \\
\text { (=Celtis spinosa Spreng; Celtis tala Gill.) }\end{array}$ & $\mathrm{Na}, \mathrm{AC}$ & $\mathrm{P}$ & Ter & 3 & 27 & 3,17 & ACE 4086 (8842), A 260 (7128) \\
\hline \multicolumn{8}{|l|}{ Chenopodiaceae (CAR) } \\
\hline Chenopodium album $\mathrm{L}$. & Ad & $\mathrm{T}$ & Ter & 7 & 25 & 3,17 & ACE 3595 (8310), A 581 (338) \\
\hline
\end{tabular}


Table 1. Continued.

\begin{tabular}{|c|c|c|c|c|c|c|c|}
\hline Taxon & Status, \# & LF & Hab & Loc & Ref $P$ & Ref I & Voucher and BAL \\
\hline $\begin{array}{l}\text { Dysphania ambrosioides (L.) Mosyakin \& Clemants } \\
\text { (= Chenopodium ambrosioides L.) }\end{array}$ & $\mathrm{Na}, \mathrm{AC}$ & $\mathrm{H}$ & Ter & 3 & 24 & 3,17 & ACE 3998 (8311), LM 689 (683) \\
\hline \multicolumn{8}{|l|}{ Cistaceae (ROS) } \\
\hline $\begin{array}{l}\text { Crocanthemum brasiliense (Lam.) Spach (= Helianthemum } \\
\text { brasiliense (Lam.) Persoon) }\end{array}$ & $\mathrm{Na}, \mathrm{SC}$ & $\mathrm{H}$ & Ter & 14 & 4 & 4,17 & ACE 3161 (8312), O 4668 (4159) \\
\hline \multicolumn{8}{|l|}{ Convolvulaceae (AST) } \\
\hline Convolvulus arvensis $\mathrm{L}$. & $\mathrm{Ad}$ & C & Ter & 7 & No & 5,17 & E 154 (8313), A 1832 (7100) \\
\hline Convolvulus hermanniae L'Hér. & $\mathrm{Na}, \mathrm{AC}$ & $\mathrm{H}$ & Ter & 13 & 5 & 5,17 & E 130 (8314), O 2301 (4472) \\
\hline Dichondra microcalyx (Hallier f.) Fabris & $\mathrm{Na}, \mathrm{AC}$ & $\mathrm{H}$ & Ter & 11 & 24 & 5,17 & $\begin{array}{l}\text { ACE } 4095(8842) \\
\text { FCA s/n (6530) }\end{array}$ \\
\hline Dichondra sericea Sw. var. sericea & $\mathrm{Na}, \mathrm{AC}$ & $\mathrm{H}$ & Ter & 11 & 5 & 5,17 & ACE 3799 (8315), O 2253 (4468) \\
\hline Evolvulus sericeus Sw. var. sericeus & $\mathrm{Na}, \mathrm{AC}$ & $\mathrm{H}$ & Ter & 20 & 5 & 5,17 & $\begin{array}{l}\text { ACE } 4028(8316), F \\
\text { CA s/n (6537) }\end{array}$ \\
\hline \multicolumn{8}{|l|}{ Crassulaceae (SAX) } \\
\hline Crassula peduncularis (Sm.) F. Meigen & $\mathrm{Na}, \mathrm{AC}$ & $\mathrm{T}$ & Ter & 18 & 26 & 3,17 & ACE 3179 (8317) \\
\hline \multicolumn{8}{|l|}{ Cucurbitaceae (ROS) } \\
\hline $\begin{array}{l}\text { Apodanthera sagittifolia (Griseb.) Mart. Crov. var. villosa (Cogn.) } \\
\text { Mart. Crov. }\end{array}$ & $\mathrm{Na}, \mathrm{SC}$ & $\mathrm{H}$ & Ter & 18 & 5 & 5,17 & ACE 4146 (8318), A 740 (410) \\
\hline \multicolumn{8}{|l|}{ Euphorbiaceae (ROS) } \\
\hline Euphorbia portulacoides L. var. portulacoides & $\mathrm{Na}, \mathrm{AC}$ & C & Ter & 13 & 23 & 4,17 & ACE 3499 (8319), O 4664 (4178) \\
\hline \multicolumn{8}{|l|}{ Fabaceae (ROS) } \\
\hline Acacia melanoxylon R. Br. & $\mathrm{Ad}$ & $P$ & Ter & 17 & 23 & $3,17,19$ & ACE 4073 (8843), FCA s/n (4979) \\
\hline Adesmia bicolor (Poir.) DC. & $\mathrm{Na}, \mathrm{AC}$ & $\mathrm{H}$ & Ter & 19 & 23 & 3,15 & ACE 3890 (8320), LM 199 (309) \\
\hline Gleditsia triacanthos L. & $\mathrm{Ad}$ & $P$ & Ter & 10 & 26 & $3,17,19$ & FCA s/n (5012) \\
\hline Lathyrus hookeri G. Don & $\mathrm{Na}, \mathrm{AC}$ & $C$ & Ter & 14 & 3 & 3,17 & ACE 4058b (8321), O 2302 (4210) \\
\hline Lathyrus nervosus Lam. & $\mathrm{Na}, \mathrm{SC}$ & $C$ & Ter & 14 & 3 & 3,17 & ACE 3730 (8322), O 3326 (4221) \\
\hline Lathyrus pubescens Hook. \& Arn. var. pubescens & $\mathrm{Na}, \mathrm{SC}$ & $C$ & Ter & 12 & 3 & 3,17 & ACE 3775 (8323), O 2320 (4216) \\
\hline Lathyrus pusillus Elliott (= L. crassipes Gillies ex Hooker \& Arnott) & $\mathrm{Na}, \mathrm{AC}$ & $\mathrm{T}$ & Ter & 11 & No & 3,17 & ACE 3773 (8324), O $4662(4218)$ \\
\hline Lathyrus tomentosus Lam. & $\mathrm{Na}, \mathrm{SC}$ & C & Ter & 12 & 3 & 3,17 & ACE 3743 (8325), O 2250 (4212) \\
\hline Lotus tenuis Waldst. \& Kit. ex Willd. & $\mathrm{Ad}$ & $\mathrm{H}$ & Ter & 7 & 28 & 3,17 & O 3665 (4225), LM 354 (225) \\
\hline Medicago arabica (L.) Huds. & $\mathrm{Ad}$ & $\mathrm{T}$ & Ter & 8 & 23 & 3,17 & FCA s/n (5095), FCA s/n (5088) \\
\hline Medicago Iupulina $\mathrm{L}$. & $\mathrm{Ad}$ & $\mathrm{T}$ & Ter & 7 & 23 & 3,17 & O 1522 (4228), NU 707 (2888) \\
\hline Medicago polymopha L. var. vulgaris (Benth.) Shinners & $\mathrm{Ad}$ & $\mathrm{T}$ & Ter & 8 & No & 3,17 & ACE 3197 (8326), A 1946 (2100) \\
\hline Melilotus officinalis (L.) Lam. & $\mathrm{Ad}$ & $\mathrm{H}$ & Ter & 18 & No & 3,17 & ACE 3623 (8327), NU 705 (2886) \\
\hline Mimosa rocae Lorentz \& Niederl. & $\mathrm{Na}, \mathrm{BA} / \#$ & $\mathrm{Ch}$ & Ter & 18 & 3 & 3,17 & ACE 3796 (8328), V 4048 (2438) \\
\hline Trifolium polymorphum Poir. var. polymorphum & $\mathrm{Na}, \mathrm{AC}$ & $\mathrm{H}$ & Ter & 13 & 26 & 3,17 & ACE 3823 (8329), A 833 (531) \\
\hline Trifolium pratense $\mathrm{L}$. & Ad & $\mathrm{H}$ & Ter & 7 & No & 3,17 & ACE 4124 (8527), LM 181 (5157) \\
\hline Trifolium repens $\mathrm{L}$. & $\mathrm{Ad}$ & $\mathrm{H}$ & Ter & 8 & 24 & 3,17 & ACE 3678 (8520), O 3279 (4248) \\
\hline Vicia graminea Sm. & $\mathrm{Na}, \mathrm{AC}$ & $\mathrm{T}$ & Ter & 13 & No & 3,17 & ACE 3741 (8330), NU 830 (6676) \\
\hline Vicia linearifolia Hook. \& Arn. & $\mathrm{Na}, \mathrm{AC}$ & $\mathrm{T}$ & Ter & 11 & No & 3,17 & ACE 3803 (8331), NU 692 (2855) \\
\hline Vicia nana Vogel & $\mathrm{Na}, \mathrm{SC}$ & $\mathrm{T}$ & Ter & 11 & 3 & 3,17 & ACE 3766 (8332), O 4661 (4256) \\
\hline Vicia setifolia Kunth. var. bonariensis Burkart & $\mathrm{Na}, \mathrm{BA} / \#$ & $\mathrm{~T}$ & Ter & 13 & 3 & 3,17 & ACE 3806 (8333) \\
\hline \multicolumn{8}{|l|}{ Fumariaceae (RAN) } \\
\hline Fumaria capreolata $\mathrm{L}$. & $\mathrm{Ad}$ & $\mathrm{T}$ & Ter & 3 & No & 3,17 & ACE 3696 (8334) \\
\hline \multicolumn{8}{|l|}{ Gentianaceae (AST) } \\
\hline Centaurium pulchellum (Sw.) Druce & $\mathrm{Ad}$ & $\mathrm{T}$ & Ter & 8 & 26 & 5,17 & ACE 3963 (8335), CL 112 (729) \\
\hline \multicolumn{8}{|l|}{ Geraniaceae (RON) } \\
\hline Geranium molle L. & $\mathrm{Ad}$ & $\mathrm{T}$ & Ter & 20 & 25 & 4,17 & ACE 3827 (8336), O 4599 (4197) \\
\hline Geranium robertianum L. & $\mathrm{Ad}$ & $\mathrm{T}$ & Ter & 21 & 4 & 4,17 & ACE 3112 (8337), O 2310 (4157) \\
\hline \multicolumn{8}{|l|}{ Hypericaceae (ROS) } \\
\hline Hypericum connatum Lam. & $\mathrm{Na}, \mathrm{AC}$ & $\mathrm{Ch}$ & Ter & 11 & 4 & 4,17 & ACE 3795 (8338), CL 1129 (6680) \\
\hline \multicolumn{8}{|l|}{ Lamiaceae (AST) } \\
\hline Ballota nigra $\mathrm{L}$. & $\mathrm{Ad}$ & $\mathrm{Ch}$ & Ter & 3 & No & 5,17 & ACE 4010 (8339) \\
\hline Lepechinia floribunda (Benth.) Epling & $\mathrm{Na}, \mathrm{AC}$ & $\mathrm{P}$ & Ter & 12 & 5 & 5,17 & ACE 3660 (8340) \\
\hline Marrubium vulgare L. & $\mathrm{Ad}$ & $\mathrm{Ch}$ & Ter & 3 & 26 & 5,17 & ACE 3984 (8341), FCA s/n (4931) \\
\hline Mentha pulegium L. & $\mathrm{Ad}$ & $\mathrm{H}$ & $\mathrm{Ma}$ & 2 & 25 & 5,17 & ACE 4035 (8342), NU 494 (4934) \\
\hline Prunella vulgaris $\mathrm{L}$. & $\mathrm{Ad}$ & $\mathrm{H}$ & $\mathrm{Ma}$ & 3 & 23 & 5,17 & ACE 4013 (8343), O 2377 (4450) \\
\hline Scutellaria racemosa Pers. & $\mathrm{Na}, \mathrm{AC}$ & $\mathrm{H}$ & Ter & 13 & 23 & 5,17 & ACE 3920 (8344), NU 4 (546) \\
\hline \multicolumn{8}{|l|}{ Lauraceae (MAG) } \\
\hline Laurus nobilis L. & $\mathrm{Ad}$ & $P$ & Ter & 3 & 27 & 19 & ACE 4105 (8846), A 1465 (7117) \\
\hline \multicolumn{8}{|l|}{ Linaceae (ROS) } \\
\hline $\begin{array}{l}\text { Cliococca selaginoides (Lam.) C. M. Rogers \& Mildner (=Linum } \\
\text { selaginoides Lam.) }\end{array}$ & $\mathrm{Na}, \mathrm{AC}$ & $\mathrm{H}$ & Ter & 11 & 4 & 4,17 & ACE 3949 (8345), O 4722 (4164) \\
\hline \multicolumn{8}{|l|}{ Loasaceae (AST) } \\
\hline Blumenbachia insignis Schard. & $\mathrm{Na}, \mathrm{AC}$ & $\mathrm{T}$ & Ter & 13 & 4 & 4,17 & O 1890 (4124), O 1890 (4124) \\
\hline
\end{tabular}


Table 1. Continued.

\begin{tabular}{|c|c|c|c|c|c|c|c|}
\hline Taxon & Status, \# & LF & Hab & Loc & Ref $P$ & Ref I & Voucher and BAL \\
\hline \multicolumn{8}{|l|}{ Lythraceae (ROS) } \\
\hline Cuphea glutinosa Cham. \& Schltdl. & $\mathrm{Na}, \mathrm{AC}$ & C & Ter & 13 & 4 & 4,17 & E 160 (8346), O 1680 (4123) \\
\hline Lythrum hyssopifolia L. & $\mathrm{Ad}$ & $\mathrm{T}$ & Ma & 1 & 25 & 4,17 & ACE 3945 (8347), V 4056 (2436) \\
\hline \multicolumn{8}{|l|}{ Malvaceae (ROS) } \\
\hline Krapovickasia flavescens (Cav.) Fryxell (=Sida flavescens Cav.) & $\mathrm{Na}, \mathrm{AC}$ & $\mathrm{H}$ & Ter & 13 & 4 & 4,17 & ACE 4049 (8348), A 426 (202) \\
\hline Modiola caroliniana (L.) G. Don & $\mathrm{Na}, \mathrm{AC}$ & $\mathrm{H}$ & Ter & 14 & 26 & 4,17 & ACE 3198 (8349), A 906 (462) \\
\hline Pavonia cymbalaria A. St.-Hil. \& Naudin & $\mathrm{Na}, \mathrm{SC}$ & $\mathrm{Ch}$ & Ter & 18 & 4 & 4,17 & ACE 4036 (8350), O 1499 (4170) \\
\hline Sida rhombifolia L. & $\mathrm{Na}, \mathrm{AC}$ & $\mathrm{Ch}$ & Ter & 11 & 23 & 4,17 & ACE 3946 (8351), A 1299 (2213) \\
\hline \multicolumn{8}{|l|}{ Montiaceae (CAR) } \\
\hline Calandrinia ciliata (Ruiz \& Pav.) DC. & $\mathrm{Na}, \mathrm{AC}$ & $\mathrm{T}$ & Ter & 21 & 3 & 3,17 & ACE 3183 (8352), O 4135 (4359) \\
\hline \multicolumn{8}{|l|}{ Myrtaceae (ROS) } \\
\hline Eucalyptus camaldulensis Dehnh. & $\mathrm{Ad}$ & $\mathrm{P}$ & Ter & 9 & 25 & 19 & ACE 4099 (8846), FCA s/n (5248) \\
\hline $\begin{array}{l}\text { Psidium salutare (Humb., Bonpl. \& Kunth) O. Berg var. } \\
\text { mucronatum (Cambess.) Landrum (=P. luridum (Spreng.) Burret) }\end{array}$ & $\mathrm{Na}, \mathrm{SC}$ & C & Ter & 13 & 23 & 4,17 & ACE 4111 (8847), CL 924 (2107) \\
\hline \multicolumn{8}{|l|}{ Oleaceae (AST) } \\
\hline Ligustrum lucidum W. T. Aiton & $\mathrm{Ad}$ & $\mathrm{P}$ & Ter & 3 & 26 & $5,17,19$ & ACE 4141 (8519) \\
\hline \multicolumn{8}{|l|}{ Onagraceae (ROS) } \\
\hline Ludwigia peploides (Kunth) P. H. Raven & $\mathrm{Na}, \mathrm{AC}$ & $\mathrm{H}$ & $\mathrm{FI}$ & 1 & 22 & 4,17 & ACE 3996 (8353), CL 284 (5225) \\
\hline Oenothera parodiana Munz & $\mathrm{Na}, \mathrm{AC}$ & $\mathrm{T}$ & Ter & 19 & 4 & 4,17 & ACE 4063 (8354) \\
\hline \multicolumn{8}{|l|}{ Orobanchaceae (AST) } \\
\hline $\begin{array}{l}\text { Agalinis communis (Cham. \& Schltdl.) D’Arcy (=Gerardia } \\
\text { communis Cham. \& Schltdl.) }\end{array}$ & $\mathrm{Na}, \mathrm{AC}$ & $\mathrm{Ch}$ & Ter & 13 & 23 & 5,17 & ACE 3940 (8355), A 566 (7155) \\
\hline $\begin{array}{l}\text { Agalinis genistifolia (Cham. \& Schltdl.) D'Arcy (=Gerardia } \\
\text { genistifolia Chas. \& Schltdl.) }\end{array}$ & $\mathrm{Na}, \mathrm{SC}$ & $\mathrm{Ch}$ & Ter & 13 & 5 & 5,17 & E 144 (8356), O 2314 (4418) \\
\hline Bartsia trixago L. (=Bellardia trixago (L.) Allioni) & $\mathrm{Ad}$ & $\mathrm{T}$ & Ter & 10 & 23 & 5,17 & ACE 3169 (8357), O 3206 (4411) \\
\hline \multicolumn{8}{|l|}{ Oxalidaceae (ROS) } \\
\hline Oxalis articulata Savigny ssp. articulata & $\mathrm{Na}, \mathrm{AC}$ & $\mathrm{C}$ & Ter & 18 & 4 & 4,17 & ACE 3804 (8358), LM 698 (690) \\
\hline Oxalis brasiliensis Lodd. (=O. macachin Arechav.) & $\mathrm{Na}, \mathrm{SC}$ & $\mathrm{C}$ & Ter & 18 & 22 & 4,17 & E 135 (8359), O 4637 (4145) \\
\hline Oxalis conorrhiza Jacq. (=O. cordobensis Knuth) & $\mathrm{Na}, \mathrm{AC}$ & $\mathrm{C}$ & Ter & 13 & 24 & 4,17 & ACE 3747 (8360), FCA s/n (6033) \\
\hline Oxalis floribunda Lehm. (=O. articulata Savigny var. hirsuta Progel) & $\mathrm{Na}, \mathrm{SC}$ & C & Ter & 13 & 4 & 4,17 & ACE 3801 (8361) \\
\hline Oxalis lasiopetala Zucc. (=O. gracillima Arechav.) & $\mathrm{Na}, \mathrm{SC}$ & $\mathrm{C}$ & Ter & 20 & 4 & 4,17 & ACE 4151 (8848), V 616 (2556) \\
\hline Oxalis perdicaria (Molina) Bertero $(=0$. mallobolba Cav.) & $\mathrm{Na}, \mathrm{SC}$ & C & Ter & 20 & 26 & 4,17 & ACE 4032 (8362), CL 1107 (6652) \\
\hline \multicolumn{8}{|l|}{ Passifloraceae (ROS) } \\
\hline Passiflora caerulea L. & $\mathrm{Na}, \mathrm{AC}$ & $\mathrm{L}$ & Ter & 3 & 4 & 4,17 & ACE 4108 (8849), A 832 (111) \\
\hline \multicolumn{8}{|l|}{ Phytolaccaceae (CAR) } \\
\hline Phytolacca dioica $\mathrm{L}$. & $\mathrm{Na}, \mathrm{AC}$ & $\mathrm{P}$ & Ter & 3 & No & 3,17 & ACE 4007 (8363) \\
\hline \multicolumn{8}{|l|}{ Plantaginaceae (AST) } \\
\hline Linaria canadensis (L.) Dum. Cours. (=L. texana Scheele) & $\mathrm{Ad}$ & $\mathrm{H}$ & Ter & 15 & 24 & 5,17 & ACE 3199 (8364), O 4667 (4426) \\
\hline Plantago myosuros Lam. & $\mathrm{Na}, \mathrm{AC}$ & $\mathrm{T}$ & Ter & 11 & 22 & 5,17 & ACE 3819 (8366), O 1530 (4385) \\
\hline $\begin{array}{l}\text { Plantago tandilensis (Pil.) Rahn =(P. brasiliense Sims var. } \\
\text { tandilensis Pilger) }\end{array}$ & $\mathrm{Na}, \mathrm{BA} / \#$ & $\mathrm{Ch}$ & Ter & 15 & 5 & 5,17 & ACE 3717 (8367), O 2288 (4382) \\
\hline Plantago tomentosa Lam. ssp. tomentosa (=P. paralias Decne) & $\mathrm{Na}, \mathrm{AC}$ & $\mathrm{H}$ & Ter & 11 & 26 & 5,17 & ACE 3909 (8368), FCA s/n (4403) \\
\hline Veronica arvensis L. & $\mathrm{Ad}$ & $\mathrm{T}$ & Ter & 7 & 26 & 5,17 & ACE 3740 (8569), O 4130 (4414) \\
\hline \multicolumn{8}{|l|}{ Polygalaceae (ROS) } \\
\hline Polygala australis A. W. Benn. & $\mathrm{Na}, \mathrm{SC}$ & $\mathrm{T}$ & Ter & 13 & 27 & 4,17 & ACE 3705 (8370), A 1487 (2377) \\
\hline Polygala linoides Poir. var. linoides Phil. & $\mathrm{Na}, \mathrm{AC}$ & $\mathrm{T}$ & Ter & 13 & 22 & $4,17,29$ & ACE 3168 (8371), NU 462 (6048) \\
\hline \multicolumn{8}{|l|}{ Polygonaceae (CAR) } \\
\hline Polygonum acuminatum Kunth var. acuminatum & $\mathrm{Na}, \mathrm{AC}$ & $\mathrm{H}$ & $\mathrm{Ma}$ & 2 & 24 & 3,17 & ACE 3994 (8372), CL 1557 (7131) \\
\hline Rumex crispus L. & $\mathrm{Ad}$ & $\mathrm{H}$ & $\mathrm{Ma}$ & 3 & 26 & 3,17 & ACE 3661 (8373), O 6161 (4314) \\
\hline Rumex obtusifolius L. & $\mathrm{Ad}$ & $\mathrm{H}$ & Ter & 19 & 23 & 3,17 & ACE 4050 (8374), 04102 (4293) \\
\hline \multicolumn{8}{|l|}{ Portulacaceae (CAR) } \\
\hline Portulaca oleracea $\mathrm{L}$. & $\mathrm{Ad}$ & $\mathrm{T}$ & Ter & 3 & No & 3,17 & O $2423(4358)$ \\
\hline Portulaca papulosa Schltdl. & $\mathrm{Na}, \mathrm{SC}$ & $\mathrm{T}$ & Ter & 18 & 3 & 3,17 & ACE 4110 (8375), A 577 (89) \\
\hline Primulaceae (AST) & & & & & & & \\
\hline Anagallis arvensis $\mathrm{L}$. & $\mathrm{Ad}$ & $\mathrm{T}$ & Ter & 13 & 22 & 5,17 & ACE 3521 (8376), O 4110 (4456) \\
\hline Centunculus minimus $\mathrm{L}$. & $\mathrm{Ad}$ & $\mathrm{T}$ & Ter & 13 & No & 5,17 & ACE 3910 (8377) \\
\hline Pelletiera verna A. St.-Hil. (=Pelletiera serpyllifolia (Poir.) Kuntze) & $\mathrm{Na}, \mathrm{AC}$ & $\mathrm{T}$ & Ter & 13 & No & 5,17 & ACE 3902 (8378) \\
\hline Ranunculaceae (RAN) & & & & & & & \\
\hline Anemone decapetala Ard. var. foliolosa Eichler & $\mathrm{Na}, \mathrm{AC}$ & C & Ter & 13 & 22 & 3,17 & E 198 (8379), CL 997 (2868) \\
\hline Rhamnaceae (ROS) & & & & & & & \\
\hline Colletia paradoxa (Spreng.) Escal. & $\mathrm{Na}, \mathrm{SC} / \#$ & $\mathrm{P}$ & Ter & 18 & 23 & 4,17 & ACE 3694a (8380), O 1657 (4148) \\
\hline Colletia spinosissima F.J. Gmel. & $\mathrm{Na}, \mathrm{AC}$ & $\mathrm{P}$ & Ter & 3 & 24 & 4,17 & ACE 3694b (8865), A 1357 (2295) \\
\hline Rosaceae (ROS) & & & & & & & \\
\hline Margyricarpus pinnatus (Lam.) Kuntze & $\mathrm{Na}, \mathrm{AC}$ & $\mathrm{Ch}$ & Ter & 18 & 22 & 3,17 & ACE 3522 (8381), NU 454 (5985) \\
\hline Prunus mahaleb L. & $\mathrm{Ad}$ & $\mathrm{P}$ & Ter & 3 & 26 & 17,19 & ACE 3174 (8382) \\
\hline
\end{tabular}


Table 1. Continued.

\begin{tabular}{|c|c|c|c|c|c|c|c|}
\hline Taxon & Status, \# & LF & Hab & Loc & Ref P & Ref I & Voucher and BAL \\
\hline Rubus ulmifolius Schott & $\mathrm{Ad}$ & $\mathrm{P}$ & Ter & 18 & No & 3,17 & ACE 4113 (8851), A 221 (5800) \\
\hline \multicolumn{8}{|l|}{ Rubiaceae (AST) } \\
\hline $\begin{array}{l}\text { Borreria dasycephala (Cham. \& Schltdl.) Bacigalupo \& E. L. } \\
\text { Cabral (=Diodia dasycephala Cham. \& Schltdl.) }\end{array}$ & $\mathrm{Na}, \mathrm{AC}$ & $\mathrm{T}$ & Ter & 13 & No & 5,17 & ACE 3936b (8383), A 769 (2386) \\
\hline $\begin{array}{l}\text { Galianthe laxa (Cham. \& Schltdl.) E. L. Cabral ssp. laxa (=Borreria } \\
\text { laxa Cham. \& Schltdl.) }\end{array}$ & $\mathrm{Na}, \mathrm{AC}$ & $\mathrm{H}$ & Ter & 2 & No & 5,17 & ACE 4252 (8384) \\
\hline Galium aparine L. & $\mathrm{Ad}$ & $\mathrm{T}$ & Ter & 3 & 23 & 5,17 & ACE 3597 (8385), O 5093 (4521) \\
\hline Galium hirtum Lam. (=Relbunium hirtum (Lam.) Schumann) & $\mathrm{Na}, \mathrm{SC}$ & $\mathrm{T}$ & Ter & 17 & 5 & 5,17 & ACE 3180 (8386), O 4665 (4518) \\
\hline $\begin{array}{l}\text { Galium hypocarpium (L.) Endl. ex Griseb. (=Relbunium } \\
\text { hypocarpium (L.) Hemsley) }\end{array}$ & $\mathrm{Na}, \mathrm{AC} / \#$ & $\mathrm{H}$ & Ter & 17 & No & 5,17 & ACE 3712 (8387) \\
\hline Sherardia arvensis $\mathrm{L}$. & $\mathrm{Ad}$ & $\mathrm{T}$ & Ter & 19 & 26 & 5,17 & ACE 4061 (8388), CL 211 (691) \\
\hline \multicolumn{8}{|l|}{ Sapindaceae (ROS) } \\
\hline Dodonaea viscosa Jacq. & Co & $\mathrm{P}$ & Ter & 18 & 24 & 4,17 & ACE 4096 (8864), O 1704 (4113) \\
\hline \multicolumn{8}{|l|}{ Scrophulariaceae (AST) } \\
\hline Buddleja thyrsoides Lam. & $\mathrm{Na}, \mathrm{SC}$ & $\mathrm{P}$ & Ter & 11 & 5 & 5,17 & ACE 3808 (8389), NU 460 (4312) \\
\hline \multicolumn{8}{|l|}{ Solanaceae (AST) } \\
\hline Cestrum parqui L'Hér. & $\mathrm{Na}, \mathrm{AC}$ & $\mathrm{P}$ & Ter & 3 & 27 & $5,12,17$ & E 132 (8390), O 1511 (4548) \\
\hline Datura ferox $\mathrm{L}$. & $\mathrm{Na}, \mathrm{AC}$ & $\mathrm{T}$ & Ter & 13 & No & $5,12,17$ & ACE 4094 (8809) \\
\hline Jaborosa runcinata Lam. & $\mathrm{Na}, \mathrm{SC}$ & C & Ter & 7 & No & $5,12,17$ & ACE 4104 (8852), CL 1099 (6640) \\
\hline Petunia axilaris (Lam.) Britton, Stern \& Poggenb. & $\mathrm{Na}, \mathrm{AC}$ & $\mathrm{T}$ & Ter & 18 & 5 & $5,12,17$ & E 157 (8391), LM 693 (696) \\
\hline Physalis viscosa $\mathrm{L}$. & $\mathrm{Na}, \mathrm{AC}$ & $C$ & Ter & 6 & 26 & $5,12,17$ & ACE 4018 (8392), LM 699 (692) \\
\hline Salpichroa origanifolia (Lam.) Baill. & $\mathrm{Na}, \mathrm{AC}$ & $\mathrm{C}$ & Ter & 4 & 5 & $5,12,17$ & E 134 (8393), A 1000 (2314) \\
\hline $\begin{array}{l}\text { Solanum chenopodioides Lam. (=S. gracilius Herter; } S \text {. } \\
\text { sublobatum Willd. ex Schult.) }\end{array}$ & $\mathrm{Na}, \mathrm{SC}$ & $\mathrm{T}$ & Ter & 11 & 5 & $5,12,17$ & ACE 3632 (8394), CL 479 (6242) \\
\hline Solanum commersonii Dunal ex Poir. & $\mathrm{Na}, \mathrm{SC}$ & $\mathrm{C}$ & Ter & 14 & 5 & $5,12,17$ & ACE 4119 (8853), NU 781 (2979) \\
\hline $\begin{array}{l}\text { Solanum granulosum-leprosum Dunal (=S. verbascifolium L. var. } \\
\text { auriculatum (Ait.) Kuntze) }\end{array}$ & $\mathrm{Na}, \mathrm{AC}$ & $\mathrm{P}$ & Ter & 3 & No & $5,12,17$ & ACE 3699 (8395), FCA s/n (6338) \\
\hline Solanum pseudocapsicum L. & $\mathrm{Na}, \mathrm{AC}$ & $\mathrm{Ch}$ & Ter & 7 & 23 & $5,12,17$ & ACE 3679 (8396), A 1859a (7108) \\
\hline Solanum sisymbriifolium Lam. & $\mathrm{Na}, \mathrm{AC}$ & $\mathrm{Ch}$ & Ter & 3 & 25 & $5,12,17$ & ACE 3697 (8397), LM 706 (723) \\
\hline \multicolumn{8}{|l|}{ Urticaceae (ROS) } \\
\hline Parietaria debilis $\mathrm{G}$. Forst. & Co & $\mathrm{T}$ & Ter & 21 & 24 & 3,17 & ACE 3185 (8398), A 765 (259) \\
\hline \multicolumn{8}{|l|}{ Verbenaceae (AST) } \\
\hline $\begin{array}{l}\text { Glandularia tenera (Spreng.) Cabrera (=G. pulchella (Sweet) } \\
\text { Tronc. var. pulchella) }\end{array}$ & $\mathrm{Na}, \mathrm{AC} / \#$ & $\mathrm{H}$ & Ter & 18 & 5 & 5,11 & ACE 3899 (8399), V 3810 (2435) \\
\hline $\begin{array}{l}\text { Phyla nodiflora (L.) Greene var. minor (=Gillies \& Hook.) N. } \\
\text { O'Leary \& Múlgura (=P. canescens (Kunth) Greene) }\end{array}$ & $\mathrm{Na}, \mathrm{AC}$ & $\mathrm{H}$ & Ter & 7 & No & 5,11 & O 1579 (4498), FCA s/n (6378) \\
\hline Verbena bonariensis L. var. bonariensis & $\mathrm{Na}, \mathrm{AC}$ & $\mathrm{T}$ & Ter & 14 & 23 & 5,11 & ACE 3967b (8400), O 16 (4489) \\
\hline Verbena intermedia Gillies \& Hook. ex Hook. & $\mathrm{Na}, \mathrm{SC}$ & $\mathrm{H}$ & Ter & 14 & 5 & 5,11 & ACE 3627 (8401), CL 238 (826) \\
\hline Verbena montevidensis Spreng. & $\mathrm{Na}, \mathrm{AC}$ & $\mathrm{T}$ & Ter & 3 & 23 & 5,11 & ACE 3641b (8402), NU 115 (757) \\
\hline \multicolumn{8}{|l|}{ Violaceae (ROS) } \\
\hline Pombalia parviflora (Mutis ex L. f.) Paula-Souza & $\mathrm{Na}, \mathrm{AC}$ & $\mathrm{H}$ & Ter & 13 & 4 & 4,17 & ACE 3193 (8403), NU 215 (6112) \\
\hline \multicolumn{8}{|l|}{ Subclass Magnoliidae (Monocotyledon species) } \\
\hline \multicolumn{8}{|l|}{ Amaryllidaceae (LIL) } \\
\hline Habranthus gracilifolius Herb. & $\mathrm{Na}, \mathrm{SC} / \#$ & $\mathrm{C}$ & Ter & 11 & 1 & 1,17 & O 4759 (4568), ACE 4102 (8854) \\
\hline $\begin{array}{l}\text { Habranthus tubispathus (L'Her.) Traub (=H. andersonii Herb.ex } \\
\text { Lindl.) }\end{array}$ & $\mathrm{Na}, \mathrm{AC}$ & $\mathrm{C}$ & Ter & 18 & 1 & 1,17 & O 3320 (4570), LM 682 (673) \\
\hline Ipheion uniflorum (Lindl.) Raf. (=Tristagma uniflorum (Lindl.) Traub) & $\mathrm{Na}, \mathrm{AC}$ & $\mathrm{C}$ & Ter & 20 & 1 & 1,17 & FCA s/n (5281), FCA s/n (5276) \\
\hline Nothoscordum gaudichaudianum Kunth & $\mathrm{Na}, \mathrm{SC}$ & $\mathrm{C}$ & Ter & 13 & No & 12,17 & ACE 3811 (8404) \\
\hline $\begin{array}{l}\text { Nothoscordum gracile (Dryand. ex Aiton) Stearn var. gracile (=N. } \\
\text { inodorum (Ait) Nicholson) }\end{array}$ & $\mathrm{Na}, \mathrm{AC}$ & $\mathrm{C}$ & Ter & 12 & 1 & 1,17 & ACE 3167 (8405), LM 658 (2406) \\
\hline $\begin{array}{l}\text { Nothoscordum montevidense Beauverd var. latitepalum (Guagl.) } \\
\text { Ravenna }\end{array}$ & $\mathrm{Na}, \mathrm{SC}$ & $\mathrm{C}$ & Ter & 13 & 24 & 1,17 & ACE 3734 (8406), LM 634 (542) \\
\hline $\begin{array}{l}\text { Nothoscordum nudicaule (Lehm.) Guagl. (=N. euosmum (Link \& } \\
\text { Otto) Kunth) }\end{array}$ & $\mathrm{Na}, \mathrm{AC}$ & $\mathrm{C}$ & Ter & 13 & 1 & 1,17 & ACE 3787 (8407), FCA s/n (5293) \\
\hline $\begin{array}{l}\text { Rodophiala bifida (Herb.) Traub (=Hippeastrum bifidum (Herb.) } \\
\text { Baker) }\end{array}$ & $\mathrm{Na}, \mathrm{AC} / \#$ & $\mathrm{C}$ & Ter & 11 & 1 & 1,17 & ACE 4112 (8408), O 4767 (4567) \\
\hline \multicolumn{8}{|l|}{ Asparagaceae (LIL) } \\
\hline Asparagus officinalis L. & $\mathrm{Ad}$ & C & Ter & 8 & No & 1,17 & ACE 3924 (8409), CL 44 (5297) \\
\hline \multicolumn{8}{|l|}{ Bromeliaceae (LIL) } \\
\hline Tillandsia bergeri Mez & $\mathrm{Na}, \mathrm{SC} / \#$ & $\mathrm{E}$ & Ter & 18 & 1 & 1,17 & ACE3966 (8410), Cl 926 (2104) \\
\hline \multicolumn{8}{|l|}{ Commelinaceae (LIL) } \\
\hline Commelina erecta L. var. angustifolia (Michx.) Fernald & $\mathrm{Na}, \mathrm{AC}$ & $\mathrm{C}$ & Ter & 11 & 1 & 1,17 & ACE 4047 (8411), Nu 108 (458) \\
\hline \multicolumn{8}{|l|}{ Cyperaceae (LIL) } \\
\hline Bulbostylis capillaris (L.) C. B. Clarke var. capillaris & $\mathrm{Na}, \mathrm{AC}$ & $\mathrm{T}$ & Ter & 18 & 1 & 1,17 & ACE 3514 (8412), A 735 (384) \\
\hline Carex bonariensis Desf. ex Poir. var. bonariensis & $\mathrm{Na}, \mathrm{AC}$ & $\mathrm{C}$ & Ter & 13 & No & 1,17 & ACE 3991 (8413), NU 26 (791) \\
\hline $\begin{array}{l}\text { Carex chilensis Brongn. ex Duperrey (=C. riparia var. chilensis } \\
\text { (Brong.) Kukenthal) }\end{array}$ & $\mathrm{Na}, \mathrm{SC}$ & $\mathrm{C}$ & $\mathrm{Ma}$ & 2 & 26 & 1,17 & ACE 3993 (8414), O 5102 (3455) \\
\hline
\end{tabular}


Table 1. Continued.

\begin{tabular}{|c|c|c|c|c|c|c|c|}
\hline Taxon & Status, \# & LF & Hab & Loc & Ref P & Ref I & Voucher and BAL \\
\hline Carex divulsa Stokes & $\mathrm{Ad}$ & $\mathrm{H}$ & Ter & 17 & No & 1,17 & ACE 4146 (8415), LM 833 (758) \\
\hline Carex phalaroides Kunth & $\mathrm{Na}, \mathrm{AC}$ & c & Ter & 8 & 1 & 1,17 & ACE 3706 (8416) \\
\hline Carex sororia Kunth ssp. sororia & $\mathrm{Na}, \mathrm{SC}$ & $\mathrm{C}$ & Ma & 2 & 26 & 1,17 & ACE 3683 (8417) \\
\hline Cyperus entrerianus Boeck. & $\mathrm{Na}, \mathrm{AC}$ & C & Ma & 2 & 28 & 1,17 & E 179 (8418), A 603 (162) \\
\hline Cyperus haspan L. var. haspan (=C. cayennensis (Lam.) Britton) & $\mathrm{Na}, \mathrm{AC}$ & c & Ma & 2 & 1 & 1,17 & ACE 4147 (8419) \\
\hline Cyperus reflexus Vahl. & $\mathrm{Na}, \mathrm{AC}$ & c & Ter & 18 & 22 & 1,17 & ACE 3645 (8420), O 2117 (3450) \\
\hline $\begin{array}{l}\text { Cyperus rigens J. Presl \& C. Presl var. serrae (Boeck) Pedersen } \\
\text { (=C. laetus ssp. laetus Presl) }\end{array}$ & $\mathrm{Na}, \mathrm{AC}$ & C & Ma & 13 & 1 & 1,17 & ACE 3675a (8421), A 604 (277) \\
\hline Eleocharis bonariensis Nees & $\mathrm{Na}, \mathrm{AC}$ & $\mathrm{H}$ & Ma & 1 & 27 & 1,17 & ACE 3989 (8422), O 3662 (3508) \\
\hline Eleocharis macrostachya Britton & $\mathrm{Na}, \mathrm{AC}$ & $\mathrm{H}$ & Ma & 3 & 26 & 1,17 & ACE 3839 (8423), O 1805 (3494) \\
\hline Eleocharis montevidensis Kunth & $\mathrm{Na}, \mathrm{AC}$ & $\mathrm{H}$ & $\mathrm{Ma}$ & 14 & 1 & 1,17 & ACE 3941 (8424), FCA s/n (6639) \\
\hline \multicolumn{8}{|l|}{ Iridaceae (LIL) } \\
\hline $\begin{array}{l}\text { Cypella herbertii (Lindl.) Herb. ssp. wolffhuegelii (Hauman) } \\
\text { Ravenna }\end{array}$ & $\mathrm{Na}, \mathrm{BA} / \#$ & C & Ter & 11 & 1 & 1,17 & O 3323 (4587), O 3323 (4587) \\
\hline Gelasine elongata (Graham) Ravenna (=G. azurea Herb.) & $\mathrm{Na}, \mathrm{SC}$ & c & Ter & 10 & 1 & 1,17 & ACE 3923 (8426), LM 660 (4780) \\
\hline $\begin{array}{l}\text { Herbertia lahue (Molina) Goldblatt ssp. amoena (Gris.) Goldblatt } \\
\text { (=Alophia lahue (Mol.) Espin. ssp amoena (Gris.) Ravenna) }\end{array}$ & $\mathrm{Na}, \mathrm{SC}$ & C & Ter & 13 & 1 & 1,17 & ACE 3825 (8427), O 2573 (4584) \\
\hline $\begin{array}{l}\text { Sisyrinchium chilense Hook (=S. iridifolium H.B.K. ssp } \\
\text { valdivianum (Phil.) Ravenna) }\end{array}$ & $\mathrm{Na}, \mathrm{AC}$ & $\mathrm{H}$ & Ter & 14 & 24 & 1,17 & ACE 3917a (8429), A 542 (69) \\
\hline Sisyrinchium minutiflorum Klatt. & $\mathrm{Na}, \mathrm{SC}$ & $\mathrm{T}$ & Ter & 18 & 12 & $1,12,17$ & ACE 3817 (8430) \\
\hline Sisyrinchium platense I. M. Johnst. & $\mathrm{Na}, \mathrm{SC}$ & $\mathrm{H}$ & Ter & 14 & 26 & $1,12,17$ & ACE 3917b (8431), CL 202 (688) \\
\hline Sisyrinchium sp. * & $\mathrm{Na}, \mathrm{SC}$ & $\mathrm{H}$ & Ter & 14 & - & $1,12,17$ & ACE 3550 (8428) \\
\hline \multicolumn{8}{|l|}{ Juncaceae (LIL) } \\
\hline Juncus balticus Willd. & $\mathrm{Na}, \mathrm{AC}$ & $\mathrm{C}$ & Ter & 14 & 23 & 1,17 & ACE 3735 (8432), NU 182 (4790) \\
\hline Juncus bufonius L. var. buffonius & $\mathrm{Na}, \mathrm{AC}$ & $T$ & Ma & 2 & 24 & 1,17 & ACE 3912 (8433), O 1817 (4612) \\
\hline Juncus capillaceus Lam. & $\mathrm{Na}, \mathrm{AC}$ & $\mathrm{H}$ & Ma & 11 & 25 & 1,17 & ACE 3688 (8434), O 1463 (4603) \\
\hline Juncus imbricatus Laharpe. & $\mathrm{Na}, \mathrm{AC}$ & $\mathrm{C}$ & Ter & 13 & 26 & 1,17 & ACE 3610b (8435), O 1997 (4611) \\
\hline Juncus microcephalus Kunth & $\mathrm{Na}, \mathrm{AC}$ & c & Ter & 3 & 1 & 1,17 & E 181 (8436), O 1959 (4599) \\
\hline Juncus pallescens Lam. (=J. dombeyanus Gay) & $\mathrm{Na}, \mathrm{AC}$ & $\mathrm{C}$ & Ma & 13 & 23 & 1,17 & ACE 3943 (8437), A 1193 (2192) \\
\hline Juncus uruguensis Griseb. & $\mathrm{Na}, \mathrm{AC} / \#$ & $\mathrm{H}$ & Ter & 18 & 23 & 1,17 & ACE 3754 (8438), A 1975 (6913) \\
\hline \multicolumn{8}{|l|}{ Lemnaceae (LIL) } \\
\hline Lemna gibba L. & Co & $\mathrm{T}$ & $\mathrm{Fl}$ & 1 & No & 1,17 & ACE 4143 (8439) \\
\hline \multicolumn{8}{|l|}{ Orchidaceae (LIL) } \\
\hline $\begin{array}{l}\text { Bipinnula pennicillata (Reichb. f.) Cisternas \& Salazar } \\
\text { (=Geoblasta pennicillata (Reichb) Hoehne ex Correa) }\end{array}$ & $\mathrm{Na}, \mathrm{SC} / \#$ & C & Ter & 14 & 1 & 1,17 & ACE 3997 (8440), A 28 (7119) \\
\hline Brachystele dilatata (Lindl.) Schltr. & $\mathrm{Na}, \mathrm{AC}$ & $\mathrm{C}$ & Ter & 17 & 1 & 1,17 & ACE 3933c (8441), NU 224 (5353) \\
\hline Chloraea membranacea Lindl. & $\mathrm{Na}, \mathrm{SC}$ & $C$ & Ter & 3 & 1 & 1,17 & FCA s/n (5356), NU 34 (150) \\
\hline \multicolumn{8}{|l|}{ Poaceae (LIL) } \\
\hline $\begin{array}{l}\text { Amelichloa brachychaeta (Godr.) Arriaga \& Barkworth (=Stipa } \\
\text { brachychaeta Godron) }\end{array}$ & $\mathrm{Na}, \mathrm{AC}$ & $\mathrm{H}$ & Ter & 10 & 28 & $2,8,17$ & ACE 3836 (8442), O 2170 (3924) \\
\hline $\begin{array}{l}\text { Aristida spegazzinii Arechav. (=A. spegazzini Arechav. var. } \\
\text { pallescens Hackel) }\end{array}$ & $\mathrm{Na}, \mathrm{AC}$ & $\mathrm{H}$ & Ter & 18 & 2 & $2,7,17$ & ACE 3501 (8443), A 1202 (1862) \\
\hline Bothriochloa laguroides (DC.) Herter var. laguroides & $\mathrm{Na}, \mathrm{AC}$ & $\mathrm{C}$ & Ter & 8 & 22 & $2,7,17$ & E 178 (8444), LM 376 (12) \\
\hline Briza maxima L. & $\mathrm{Ad}$ & $\mathrm{T}$ & Ter & 11 & 23 & $2,8,17$ & O 2149 (4027), NU 458 (1089) \\
\hline Briza minor $\mathrm{L}$. & $\mathrm{Ad}$ & $\mathrm{T}$ & Ter & 21 & 22 & $2,8,17$ & ACE 3791 (8445), O 1690 (4030) \\
\hline $\begin{array}{l}\text { Bromidium tandilense (Kuntze) Rúgolo (=Agrostis tandilensis } \\
\text { (Kuntze) Parodi) }\end{array}$ & $\mathrm{Na}, \mathrm{AC}$ & $\mathrm{T}$ & Ter & 18 & No & $2,8,17$ & ACE 3716 (8446), A 1442 (1576) \\
\hline Bromus auleticus Trin. ex Nees & $\mathrm{Na}, \mathrm{AC}$ & $\mathrm{H}$ & Ter & 11 & 23 & $2,8,17$ & ACE 3863 (8447), CL 307 (1056) \\
\hline $\begin{array}{l}\text { Bromus catharticus Vahl var. catharticus (=B. unioloides } \\
\text { Humboldt) }\end{array}$ & $\mathrm{Na}, \mathrm{AC}$ & $\mathrm{H}$ & Ter & 17 & 23 & $2,8,17$ & ACE 3784 (8448), CL 995 (6954) \\
\hline Bromus hordaceus L. (=B. mollis L.) & $\mathrm{Ad}$ & $\mathrm{T}$ & Ter & 13 & 26 & $2,8,17$ & ACE 3886 (8449), CL 1037 (2816) \\
\hline $\begin{array}{l}\text { Chascolytrum brizoides (Lam.) Essi, Longhi-Wagner \& Souza- } \\
\text { Chies (=Briza brizoides (Lam.) Kuntze) }\end{array}$ & $\mathrm{Na}, \mathrm{AC}$ & $\mathrm{H}$ & Ter & 20 & 2 & $2,8,17$ & ACE 3897 (8450), NU 459 (577) \\
\hline Chascolytrum rufum J. Presl (=Briza rufa (Presl) Steudel) & $\mathrm{Na}, \mathrm{AC}$ & $\mathrm{H}$ & Ter & 4 & 22 & $2,8,17$ & ACE 3919 (8451), O 4677 (4013) \\
\hline Chascolytrum subaristatum (Lam.) Desv. (=Briza subaristata Lam.) & $\mathrm{Na}, \mathrm{AC}$ & $\mathrm{H}$ & Ter & 18 & 2 & $2,8,17$ & ACE 3715 (8452), LM 540 (190) \\
\hline $\begin{array}{l}\text { Chascolytrum uniolae (Ness) Essi, Longhi-Wagner \& Souza- } \\
\text { Chies (=Briza uniolae (Nees) Ness ex Steudel) }\end{array}$ & $\mathrm{Na}, \mathrm{AC}$ & $\mathrm{H}$ & Ter & 19 & 2 & $2,8,17$ & ACE 3539 (8453), LM 558 (147) \\
\hline Cortaderia selloana (Schult. \& Schult. f.) Asch. \& Graebn. & $\mathrm{Na}, \mathrm{AC}$ & $\mathrm{H}$ & Ter & 11 & 22 & $2,7,17$ & ACE 4089 (8505), A 450 (224) \\
\hline Cynodon dactylon (L.) Pers. & $\mathrm{Ad}$ & $\mathrm{C}$ & Ter & 7 & 26 & $2,7,17$ & ACE 4092 (8506), O 2195 (4005) \\
\hline Cynosurus echinatus L. & $\mathrm{Ad}$ & $\mathrm{T}$ & Ter & 4 & 23 & $2,8,17$ & ACE 4054 (8454), NU 222 (1252) \\
\hline Dactylis glomerata L. & $\mathrm{Ad}$ & $\mathrm{T}$ & Ter & 17 & 26 & $2,8,17$ & ACE 4093 (8511), CL 310 (1576) \\
\hline Danthonia cirrata Hack. \& Arechav. & $\mathrm{Na}, \mathrm{AC}$ & $\mathrm{C}$ & Ter & 10 & 2 & $2,7,17$ & ACE 3535 (8455), NU 327 (1287) \\
\hline Danthonia montevidensis Hack. \& Arechav. & $\mathrm{Na}, \mathrm{SC} / \#$ & $\mathrm{H}$ & Ter & 18 & No & $2,7,17$ & ACE 4029 (8456), O 2143 (3801) \\
\hline Deyeuxia alba J. Presl ssp. alba (=D. armata (Doell) Parodi) & $\mathrm{Na}, \mathrm{AC}$ & $\mathrm{C}$ & Ter & 13 & 2 & $2,8,17$ & ACE 3845 (8457), LM 651 (269) \\
\hline Deyeuxia viridiflavescens (Poir.) Kunh & $\mathrm{Na}, \mathrm{AC}$ & $\mathrm{C}$ & Ter & 18 & 2 & $2,8,17$ & ACE 3483 (8458), O 5233 (3790) \\
\hline $\begin{array}{l}\text { Dichanthelium sabulorum (Lam.) Gould \& C.A. Clark. (=Panicum } \\
\text { sabulorum Lam.) }\end{array}$ & $\mathrm{Na}, \mathrm{AC}$ & $\mathrm{H}$ & Ter & 11 & 2 & $2,7,17$ & ACE 3638 (8459), O 5197 (3564) \\
\hline
\end{tabular}


Table 1. Continued.

\begin{tabular}{|c|c|c|c|c|c|c|c|}
\hline Taxon & Status, \# & LF & Hab & Loc & Ref $P$ & Ref I & Voucher and BAL \\
\hline Echinochloa crus-galli (L.) P. Beauv. var. crus-galli & $\mathrm{Ad}$ & $\mathrm{T}$ & $\mathrm{Ma}$ & 2 & 26 & $2,7,17$ & A 1766 (1927), NU 404 (1309) \\
\hline Echinochloa crus-galli (L.) P. Beauv. var. mitis (Pursh.) Peterm. & $\mathrm{Ad}$ & $\mathrm{T}$ & $\mathrm{Ma}$ & 2 & No & $2,7,17$ & ACE 4097 (8460) \\
\hline Echinochloa crus-pavonis (Kunth) Schultz. & $\mathrm{Ad}$ & $\mathrm{T}$ & $\mathrm{Ma}$ & 2 & No & $2,7,17$ & ACE 3682 (8507) \\
\hline Eleusine indica (L.) Gaertn & $\mathrm{Ad}$ & $\mathrm{T}$ & Ter & 11 & No & $2,7,17$ & ACE 4098 (8508), V 4066 (1804) \\
\hline Elionurus muticus (Spreng.) Kuntze & $\mathrm{Na}, \mathrm{AC}$ & $\mathrm{H}$ & Ter & 18 & 2 & $2,7,17$ & ACE 3891 (8461), V 3627 (2539) \\
\hline Eragrostis airoides Nees & $\mathrm{Na}, \mathrm{AC}$ & $\mathrm{H}$ & Ter & 13 & 2 & $2,7,17$ & E 156 (8462), V 3928 (1812) \\
\hline Eragrostis lugens Nees & $\mathrm{Na}, \mathrm{AC}$ & $\mathrm{H}$ & Ter & 20 & 22 & $2,7,17$ & ACE 3649 (8463), A 733 (397) \\
\hline Festuca arundinacea Schreb. & $\mathrm{Ad}$ & $\mathrm{C}$ & Ter & 17 & 2 & $2,8,17$ & ACE 3985 (8464), CL 314 (1636) \\
\hline Glyceria multiflora Steud. & $\mathrm{Na}, \mathrm{AC}$ & $\mathrm{H}$ & Ma & 1 & 22 & $2,8,17$ & ACE 3999 (8465), NU 7 (397) \\
\hline Holcus lanatus $\mathrm{L}$. & $\mathrm{Ad}$ & $\mathrm{T}$ & Ter & 6 & 24 & $2,8,17$ & E 176 (8466), O 2150 (3602) \\
\hline $\begin{array}{l}\text { Hordeum murinum L. ssp. leporinum (Link) Arcang. (=H. } \\
\text { leporinum Link.) }\end{array}$ & $\mathrm{Ad}$ & $\mathrm{T}$ & Ter & 13 & 23 & $2,8,17$ & O 5136 (3607), LM 536 (1371) \\
\hline Hordeum stenostachys Godr. & $\mathrm{Na}, \mathrm{AC}$ & $\mathrm{H}$ & Ter & 18 & 26 & $2,8,17$ & ACE 3484 (8467), CL 205 (845) \\
\hline Jarava filifolia (Nees.) Ciald. (=Stipa filifolia Nees) & $\mathrm{Na}, \mathrm{SC}$ & $\mathrm{H}$ & Ter & 13 & 2 & $2,8,17$ & ACE 4145 (8468), CL 189 (1867) \\
\hline Jarava juncoides (Speg.) Peñailillo (=Stipa juncoides Speg.) & $\mathrm{Na}, \mathrm{SC}$ & $\mathrm{H}$ & Ter & 13 & 2 & $2,8,17$ & ACE 3556 (8469), CL 177 (737) \\
\hline Leersia hexandra Sw. & $\mathrm{Na}, \mathrm{AC}$ & $\mathrm{C}$ & $\mathrm{Ma}$ & 1 & 22 & $2,7,17$ & ACE 3992 (8470), A 1258 (1379) \\
\hline Lolium multiflorum Lam. & $\mathrm{Ad}$ & $\mathrm{T}$ & Ter & 8 & 24 & $2,8,17$ & ACE 3976 (8471), CL 263 (1385) \\
\hline Lolium perenne L. & $\mathrm{Ad}$ & $\mathrm{H}$ & Ter & 8 & No & $2,8,17$ & ACE 3829 (8472), CL 201 (1381) \\
\hline Melica brasiliana Ard. & $\mathrm{Na}, \mathrm{SC}$ & C & Ter & 20 & 2 & $2,8,17$ & ACE 3534 (8473), LM 543 (38) \\
\hline Melica macra Nees & $\mathrm{Na}, \mathrm{SC}$ & $\mathrm{C}$ & Ter & 13 & 26 & $2,8,17$ & ACE 4065 (8475), O 5217 (3647) \\
\hline Melica parodiana Torres & $\mathrm{Na}, \mathrm{SC} / \#$ & $\mathrm{C}$ & Ter & 18 & 2 & $2,8,17$ & ACE 3930 (8476) \\
\hline $\begin{array}{l}\text { Melica rigida Cav. (M. aurantiaca Desr. ex Lam. var. rigida (Cav.) } \\
\text { Papp) }\end{array}$ & $\mathrm{Na}, \mathrm{SC}$ & C & Ter & 10 & No & 8,17 & ACE 3761 (8474) \\
\hline Nassella filiculmis Barkworth (=Stipa filiculmis Delile) & $\mathrm{Na}, \mathrm{SC}$ & $\mathrm{H}$ & Ter & 13 & 2 & $2,8,17$ & ACE 3889 (8477), O 7159 (4683) \\
\hline $\begin{array}{l}\text { Nassella megapotamia Barkworth (=Stipa megapotamia } \\
\text { Spreng. ex Trin.) }\end{array}$ & $\mathrm{Na}, \mathrm{SC}$ & $\mathrm{H}$ & Ter & 11 & 2 & $2,8,17$ & ACE 3831 (8478), A 1225 (1520) \\
\hline $\begin{array}{l}\text { Nassella melanosperma Barkworth (=Stipa melanosperma } \\
\text { J. Presl) }\end{array}$ & $\mathrm{Na}, \mathrm{AC}$ & $\mathrm{H}$ & Ter & 11 & 24 & $2,8,17$ & ACE 3881 (8479), O 5283 (4669) \\
\hline Nassella neesiana Barkworth (=Stipa neesiana Trin. \& Rupr.) & $\mathrm{Na}, \mathrm{AC}$ & $\mathrm{H}$ & Ter & 11 & 22 & $2,8,17$ & E 98 (8480), O 5106 (3900) \\
\hline Panicum bergii Arechav. & $\mathrm{Na}, \mathrm{AC}$ & $\mathrm{H}$ & Ter & 18 & 26 & $2,7,17$ & E 93 (8481), CL 232 (687) \\
\hline Paspalum dilatatum Poir. ssp. dilatatum & $\mathrm{Na}, \mathrm{AC}$ & $\mathrm{C}$ & Ter & 8 & 26 & $2,7,17$ & ACE 4106 (8509), NU 398 (1165) \\
\hline Paspalum distichum $\mathrm{L}$. & $\mathrm{Na}, \mathrm{AC}$ & $\mathrm{C}$ & $\mathrm{Ma}$ & 2 & 26 & $2,7,17$ & ACE 4004 (8482), A 744 (314) \\
\hline Paspalum exaltatum J. Presl & $\mathrm{Na}, \mathrm{SC}$ & $\mathrm{C}$ & Ter & 5 & 22 & $2,7,17$ & ACE 3951 (8483), A 1589 (6752) \\
\hline Paspalum plicatulum Michx. & $\mathrm{Na}, \mathrm{AC}$ & $\mathrm{C}$ & Ter & 14 & 26 & $2,7,17$ & ACE 3625 (8484), V 3945 (1816) \\
\hline Paspalum quadrifarium Lam. & $\mathrm{Na}, \mathrm{SC}$ & $\mathrm{C}$ & Ter & 14 & 2 & $2,7,17$ & ACE 3693 (8485), LM 687 (1133) \\
\hline Phalaris angusta Nees ex Trin. & $\mathrm{Na}, \mathrm{AC}$ & $\mathrm{T}$ & Ter & 12 & 22 & $2,8,17$ & ACE 3758 (8486), O 5294 (4654) \\
\hline Phalaris aquatica L. & $\mathrm{Ad}$ & $\mathrm{C}$ & Ter & 4 & No & $2,8,17$ & ACE 3832 (8487), NU 356 (1410) \\
\hline Piptochaetium bicolor (Vahl) E. Desv. & $\mathrm{Na}, \mathrm{SC}$ & $\mathrm{H}$ & Ter & 18 & 22 & $2,8,17$ & E 96 (8488), LM 542 (135) \\
\hline Piptochaetium lasianthum Griseb. & $\mathrm{Na}, \mathrm{SC}$ & $\mathrm{H}$ & Ter & 18 & 2 & $2,8,17$ & ACE 3860 (8489), O 3224 (3732) \\
\hline Piptochaetium montevidense (Spreng.) Parodi & $\mathrm{Na}, \mathrm{AC}$ & $\mathrm{H}$ & Ter & 18 & 2 & $2,8,17$ & ACE 3847 (8490), LM 745 (198) \\
\hline Piptochaetium ruprechtianum E. Desv. & $\mathrm{Na}, \mathrm{SC}$ & $\mathrm{H}$ & Ter & 18 & 2 & $2,8,17$ & ACE 3862 (8491), LM 541 (58) \\
\hline $\begin{array}{l}\text { Piptochaetium stipoides (Trin. \& Rupr.) Hack. ex Arechav. var. } \\
\text { stipoides }\end{array}$ & $\mathrm{Na}, \mathrm{AC}$ & $\mathrm{H}$ & Ter & 18 & 22 & $2,8,17$ & ACE 3882 (8492), O 5266 (4680) \\
\hline Poa bonariensis (Lam.) Kunth (=P. montevidensis Arechav.) & $\mathrm{Na}, \mathrm{AC}$ & $\mathrm{C}$ & Ter & 8 & 23 & $2,8,17$ & ACE 3839 (8493), O 5198 (3816) \\
\hline Poa compressa L. & $\mathrm{Ad}$ & $\mathrm{C}$ & Ter & 3 & No & $2,8,17$ & ACE 3536 (8494) \\
\hline Poa iridifolia Hauman & $\mathrm{Na}, \mathrm{BA} / \#$ & $\mathrm{H}$ & Ter & 15 & 2 & $2,8,17$ & ACE 3965 (8495), V 3756 (1811) \\
\hline Poa lanigera Nees & $\mathrm{Na}, \mathrm{AC}$ & $\mathrm{H}$ & Ter & 13 & No & $2,8,17$ & ACE 3738 (8496), NU 20 (1011) \\
\hline Poa lanuginosa Poir. & $\mathrm{Na}, \mathrm{AC}$ & $\mathrm{C}$ & Ter & 13 & 2 & $2,8,17$ & ACE 3749 (8497), O 2350 (3820) \\
\hline Poa ligularis Nees ex Steud var. ligularis & $\mathrm{Na}, \mathrm{SC}$ & $\mathrm{H}$ & Ter & 13 & 2 & $2,8,17$ & ACE 3802 (8598), CL 1000 (2874) \\
\hline $\begin{array}{l}\text { Poa ligularis Nees ex Steud var. resinulosa (Ness ex Steud.) } \\
\text { Fernández Pepi \& Giussani (=P. resinulosa Nees ex Steud.) }\end{array}$ & $\mathrm{Na}, \mathrm{SC}$ & $\mathrm{H}$ & Ter & 11 & 2 & $2,8,17$ & ACE 3163 (8599), LM 957 (1427) \\
\hline Schizachyrium spicatum (Spreng.) Herter & $\mathrm{Na}, \mathrm{AC}$ & $\mathrm{H}$ & Ter & 18 & 22 & $2,7,17$ & ACE 3895 (8500), V 4028 (1810) \\
\hline $\begin{array}{l}\text { Setaria parviflora (Poir.) Kerguélen var. parviflora (=S. geniculata } \\
\text { (Lam.) Beauvois) }\end{array}$ & $\mathrm{Na}, \mathrm{AC}$ & $\mathrm{C}$ & Ter & 18 & 23 & $2,7,17$ & E 152 (8501), CL 364 (1479) \\
\hline Setaria vaginata Spreng. var. bonariensis Nicora & $\mathrm{Na}, \mathrm{SC}$ & $\mathrm{H}$ & Ter & 11 & 2 & $2,7,17$ & ACE 4009 (8502) \\
\hline Sorghastrum pellitum (Hack.) Parodi & $\mathrm{Na}, \mathrm{AC}$ & $\mathrm{H}$ & Ter & 14 & 23 & $2,7,17$ & E 101 (8503), O 3253 (3880) \\
\hline Sporobolus indicus (L.) R. Br. & $\mathrm{Na}, \mathrm{AC}$ & $\mathrm{H}$ & Ter & 18 & 23 & $2,7,17$ & ACE 3636 (8504), LM 338 (21) \\
\hline Vulpia australis (Ness ex Steud.) C.H. Blom & $\mathrm{Na}, \mathrm{SC}$ & $\mathrm{T}$ & Ter & 18 & 26 & $2,8,17$ & ACE 4059 (8510), O 5181 (3871) \\
\hline Vulpia bromoides (L.) Gray (=V. dertonensis (All.) Gola) & $\mathrm{Ad}$ & $\mathrm{T}$ & Ter & 18 & 2 & $2,8,17$ & ACE 3755 (8512), NU 23 (669) \\
\hline Vulpia muralis (Kunth) Nees & $\mathrm{Ad}$ & $\mathrm{T}$ & Ter & 18 & No & 8,17 & ACE 3957 (8515) \\
\hline Vulpia myuros (L.) C. C. Gmel. f. megalura (Nutt.) Auquier (= & $\mathrm{Ad}$ & $\mathrm{T}$ & Ter & 13 & 26 & $2,8,17$ & ACE 3986 (8513), O 5213 (3876) \\
\hline
\end{tabular}
megalura (Nutt.) Rydberg)

Typhaceae (LIL)

Typha latifolia L. 
Table 2. Site identifiers correspond to labels in Figure 3. The spatial references are geographic coordinates relative to the WGS84 datum.

\begin{tabular}{llll}
\hline Site & Habitat remarks & Latitude (S) & Longitude (W) \\
\hline 1 & Pond and stream & $37^{\circ} 55^{\prime} 26.12^{\prime \prime}$ & $057^{\circ} 49^{\prime} 19.60^{\prime \prime}$ \\
2 & Pond and stream & $37^{\circ} 55^{\prime} 25.97^{\prime \prime}$ & $057^{\circ} 49^{\prime} 18.95^{\prime \prime}$ \\
3 & Shaded banks of the stream & $37^{\circ} 55^{\prime} 27.47^{\prime \prime}$ & $057^{\circ} 49^{\prime} 19.76^{\prime \prime}$ \\
4 & Arboreal stratum & $37^{\circ} 55^{\prime} 30.93^{\prime \prime}$ & $057^{\circ} 49^{\prime} 20.36^{\prime \prime}$ \\
5 & Intermediate slope & $37^{\circ} 55^{\prime} 25.62^{\prime \prime}$ & $057^{\circ} 49^{\prime} 22.67^{\prime \prime}$ \\
6 & Grassland & $37^{\circ} 55^{\prime} 24.63^{\prime \prime}$ & $057^{\circ} 49^{\prime} 20.63^{\prime \prime}$ \\
7 & Grassland & $37^{\circ} 55^{\prime} 26.89^{\prime \prime}$ & $057^{\circ} 49^{\prime} 17.00^{\prime \prime}$ \\
8 & Grassland & $37^{\circ} 55^{\prime} 28.79^{\prime \prime}$ & $057^{\circ} 49^{\prime} 18.56^{\prime \prime}$ \\
9 & Grassland & $37^{\circ} 55^{\prime} 39.34^{\prime \prime}$ & $057^{\circ} 49^{\prime} 04.42^{\prime \prime}$ \\
10 & Valley of the E summit & $37^{\circ} 55^{\prime} 33.55^{\prime \prime}$ & $057^{\circ} 49^{\prime} 15.00^{\prime \prime}$ \\
11 & Lower slope & $37^{\circ} 55^{\prime} 38.76^{\prime \prime}$ & $057^{\circ} 49^{\prime} 16.52^{\prime \prime}$ \\
12 & Upper slope & $37^{\circ} 55^{\prime} 36.04^{\prime \prime}$ & $057^{\circ} 49^{\prime} 19.09^{\prime \prime}$ \\
13 & Upper slope & $37^{\circ} 55^{\prime} 39.71^{\prime \prime}$ & $057^{\circ} 49^{\prime} 12.61^{\prime \prime}$ \\
14 & Rocky, grassland slope & $37^{\circ} 55^{\prime} 39.43^{\prime \prime}$ & $057^{\circ} 49^{\prime} 10.68^{\prime \prime}$ \\
15 & Humid, grassland slope & $37^{\circ} 55^{\prime} 33.23^{\prime \prime}$ & $057^{\circ} 49^{\prime} 12.38^{\prime \prime}$ \\
16 & NE summit & $37^{\circ} 55^{\prime} 36.64^{\prime \prime}$ & $057^{\circ} 49^{\prime} 10.25^{\prime \prime}$ \\
17 & Valley in the N summit & $37^{\circ} 55^{\prime} 39.89^{\prime \prime}$ & $057^{\circ} 49^{\prime} 07.93^{\prime \prime}$ \\
18 & "Acacias" area & $37^{\circ} 55^{\prime} 40.26^{\prime \prime}$ & $057^{\circ} 49^{\prime} 05.53^{\prime \prime}$ \\
19 & Central summit & $37^{\circ} 55^{\prime} 40.47^{\prime \prime}$ & $057^{\circ} 49^{\prime} 03.84^{\prime \prime}$ \\
20 & Valley in the SE summit & $37^{\circ} 55^{\prime} 43.37^{\prime \prime}$ & $057^{\circ} 49^{\prime} 00.87^{\prime \prime}$ \\
21 & SE summit & $37^{\circ} 55^{\prime} 31.87^{\prime \prime}$ & $057^{\circ} 49^{\prime} 11.92^{\prime \prime}$ \\
\hline & & & \\
\hline & & & \\
\hline & & &
\end{tabular}

\section{Results}

Identified taxa in the inventoried area of Paititi Natural Reserve are presented in Table 1. Some species were only found in specific sites of the reserve, while others were found in many different sites. The location where each species was more frequently found is described in Fig. 3 and detailed in Tables 1 and 2. In the untouched area the richness of vascular plants reached 364 species and infraspecific taxa. In the untouched area the richness of vascular plants reached 360 species and 4 species were represented by 2 varieties each (Conyza bonariensis, $C$. sumatrensis, Echinochloa crus-galli, and Poa ligularis). One Sisyrinchium sp. could not be identified to species.

Of all recorded species, $97.5 \%$ belong to the Spermatophytes (subclasses Pinidae and Magnoliidae), and 2.5\% to the Polypodiidae (Pteridaceae, with 3 species, the best represented family). Among the Spermatophytes, only 2 species of Subclass Pinidae were found, while among Magnoliidae (angiosperms), 234 species and infraspecific taxa belong to basal dicot and eudicot superorders (64.3\%) and 119 to the monocot superorder $(32.7 \%)$. The recorded vascular plants belong to 72 families, with 34 families represented with only 1 specific taxon and 11 with 2 taxa (Table 1). Six families had more than 10 taxa each, Poaceae with 76 taxa (20.9\%), Asteraceae with 73 (20.1\%), Fabaceae with 21 (5.8\%), Cyperaceae with 13 (3.6\%), and Apiaceae and Solanaceae with 11 taxa each (3.0\%). The genera of subclass Magnoliidae that contributed with most species were: Gamochaeta (8), Juncus (7), Baccharis, Oxalis and Poa (6), Carex, Eryngium, Lathyrus, Paspalum, Piptochaetium and Solanum (5), and Chascolytrum, Cyperus, Hypochaeris, Melica, Nassella, Sisyrinchium, Senecio, Vicia and Vulpia (4).

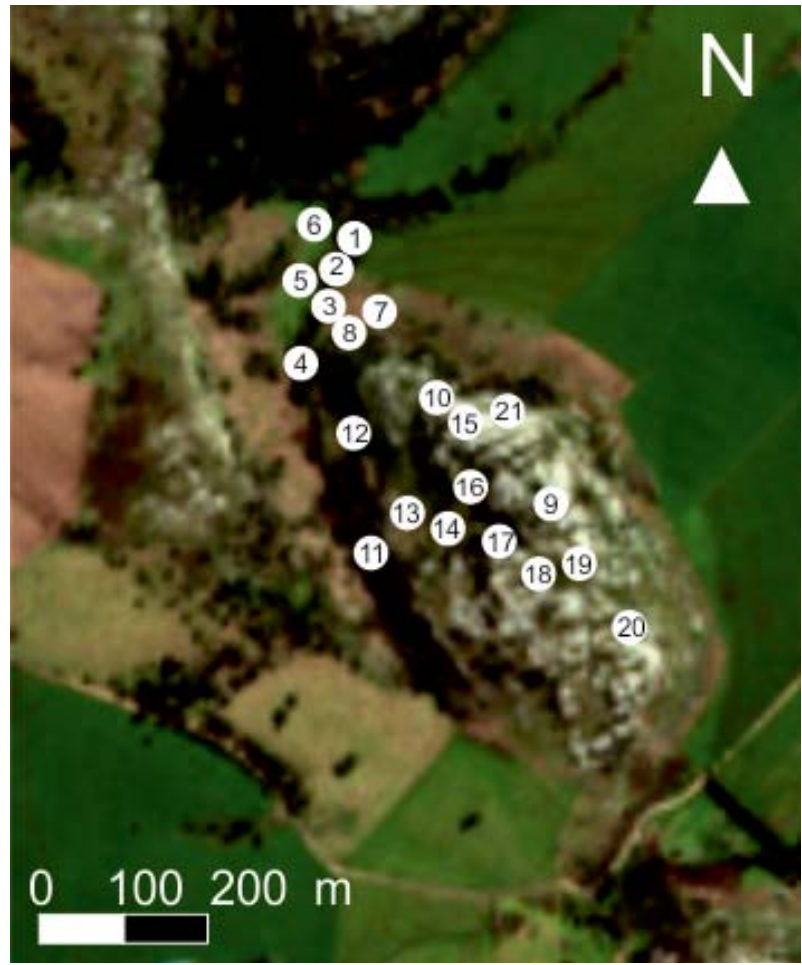

Figure 3. Location. Each number corresponds to a location where certain species were more frequently found. This figure complements tables 1 and 2. Satellite image obtained from Sentinel Playground (Sentinel Playground, 2017).

Regarding the geographic origin of the species and the infraspecific taxa, $1.1 \%$ were cosmopolitan, $26.1 \%$ were adventive, and $72.8 \%$ were native (Fig. 4 ). Within this last group, 187 species grow in several places of the American continent, 78 species occur exclusively in the Southern Cone Region of South America (Flora Argentina 2017), and only 7 species are endemic to mountain ranges of Buenos Aires province (Table 1). From the total number of taxa, $30.6 \%$ were annual or biennial plants (therophytes), while $69.4 \%$ were perennial species. Among the perennial species, the most abundant life forms were hemicryptophytes (33.1\%) and cryptophytes $(22.6 \%)$. Lianas and epiphytes phanerophytes, chamaephytes, and phanerophytes were scarce $(1.1 \%, 6.1 \%$, $6.6 \%$, respectively). Each life form was mainly represented by species of native status, except for therophytes, most of which were adventive (Fig. 4).

Diagnoses, descriptions, and images are presented for 1 species that was not fully identified, 10 species that

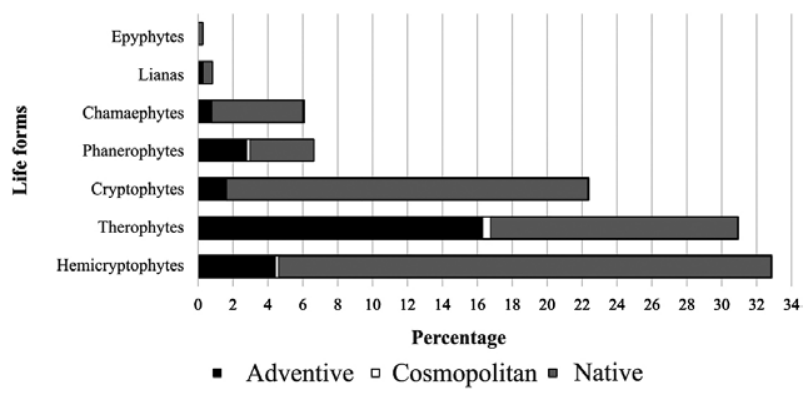

Figure 4. Frequency of different life forms of vascular plant according to its status in the Paititi Natural Reserve. 
were newly recorded in Buenos Aires mountain ranges, and 31 native species, mostly threatened according to Delucchi (2006).

Subclass Polypodiidae

Order Polypodiales: Family Pteridaceae: Subfamily Vittarioideae

\section{Genus Adiantum L.}

\section{Adiantum raddianum C. Presl.}

Figure 5

Adiantum raddianum C. Presl (1836): 158 - Cabrera (1968): 178; Flora Argentina (2017); Tropicos (2017).

Adiantum cuneatum Langsdorff \& Fischer (1810): 23—Capurro (1961): 121.

Cryptophyte, native with wide distribution in the American continent. Least Concern (Delucchi 2006).

Characteristics. Delicate fern; rhizomes cylindrical, covered with brown-yellowish scales. Fronds triangular, pinnately compound, glabrous, with a thin, cylindrical, dark, almost black, glossy petiole. Pinnules alternate, irregular, almost rhombic; apex incised, with base cuneate, veins dichotomous; petioles long, thread-like. Sorus located on edge of pinnules.

Comments. This is a rare species in the study area. This fern grows in shady, humid sites. At the summit, it grows in deep cracks of rocks and also along the shaded banks of the stream. The major difference between $A$. raddianum and $A$. chilense Kaulfuss, which is also frequent in Buenos Aires mountains, is in the shape of the pinnules; the latter species presents reniform pinnules without cuneate bases and with the upper margin entire or with short incisions.

Subclass Magnoliidae

Superorder Lilianae

Order Asparagales: Family Amaryllidaceae: Tribe Gilliesiae

\section{Genus Nothoscordum Kunth}

\section{Nothoscordum gaudichaudianum Kunth}

Figure 6

Nothoscordum gaudichaudianum Kunth (1843): 458-Hurrell (2009b): 44; Flora Argentina (2017); Tropicos (2017).

Nothoscordum grossibulbum Beauverd (1908): 1003.

Allium bivalve (L.) Kuntze var. gaudichaudianum (Kunth) Kuntze (1898): 312-Guaglianone (1972): 159-242. Allium bivalve (L.) Kuntze (1898): 315.

Cryptophyte, native with distribution in the Southern Cone Region of South America; previosly known from the Entre Ríos and Corrientes provinces (Argentina) but not from Buenos Aires province.

Characteristics. Perennial herb to $25 \mathrm{~cm}$ tall. Bulb subglobose, $1.5 \mathrm{~cm}$ in diameter, with onion odor, without lateral rhizomes. Leaves linear, 1-3 cm long, veins parallel, apex obtuse, 4-18 cm long. Peduncles longer than foliage. Inflorescence: an umbel, with 2-5 flowers, with erect, unequal pedicels; rotate perianth with 6 white tepals with purplish tinge; free filaments. Fruit: a capsule.

Comments. This is a rare species in the study area.
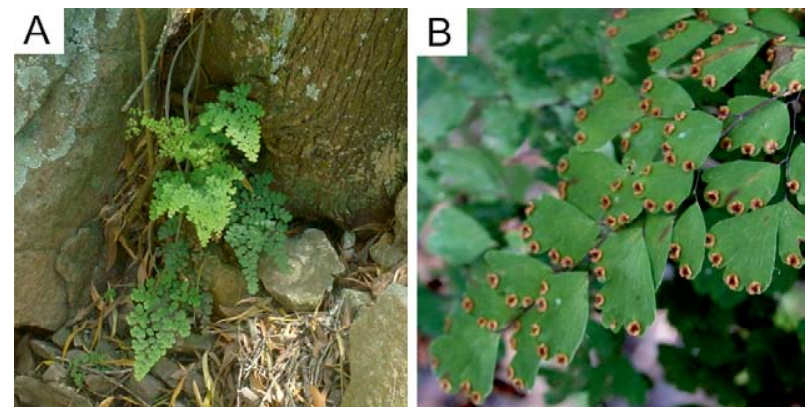

Figure 5. Adiantum raddianum. A. Plant. B. Detail of the sorus that are located on the edge of the pinnules.

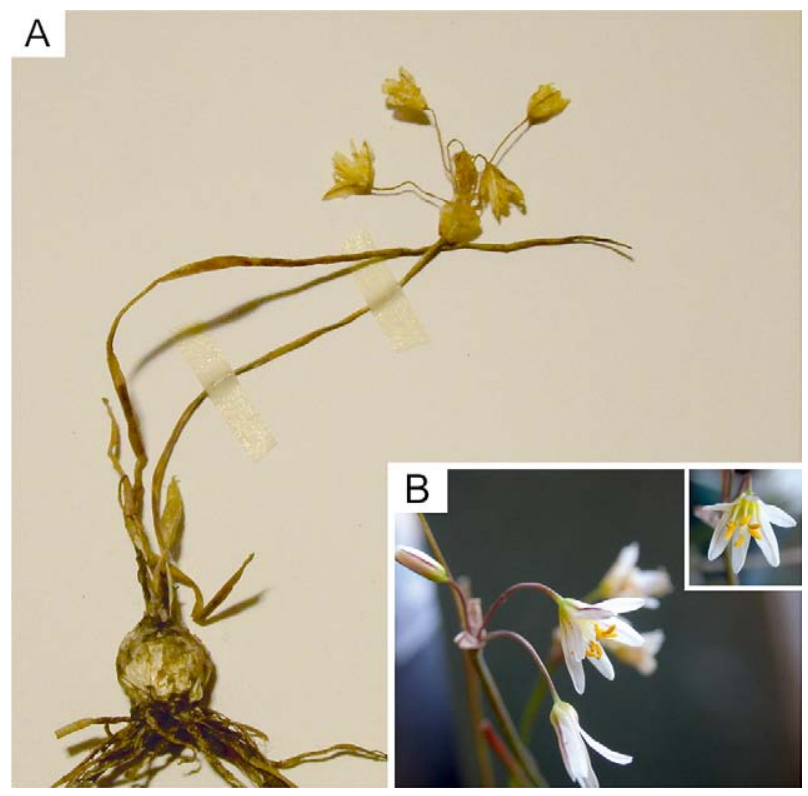

Figure 6. Nothoscordum gaudichaudianum. A. Plant. B. Inflorescence and flower detail.

It grows in full sunlight conditions on shallow to moderately deep, damp soils on rocky and grassy slopes with other small to medium-sized species. Nothoscordum gaudichaudianum can be distinguished from other Nothoscordum species by its erect peduncles, white tepals, free filaments, and bulbs with characteristic onion odor.

\section{Nothoscordum montevidense Beauv. ssp. latitepalum (Guag.) Ravenna}

Figure 7

Nothoscordum montevidense Beauv. var. latitepalum (Guag.) Ravenna (1978): 144-Hurrell (2009b): 49; Flora Argentina (2017); Tropi$\cos (2017)$.

Nothoscordum montevidense Beauv. var. latipetalum Guaglianone (1972): 232.

Nothoscordum montevidense Beauverd (1906): 1011 - Cabrera (1968): 515.

Cryptophyte, native with distribution in the Southern Cone Region of South America; previously recorded from Buenos Aires province but not from mountainous areas.

Characteristics. Perennial herb, 5-10 cm tall. Bulb solitary, globose, ca $1 \mathrm{~cm}$ in diameter. Sheaths short. Leaves to $10 \mathrm{~cm}$ long, parallel-veined, linear, with apex acute. Peduncles erect. Inflorescence: an umbel, with 2-10 flowers. Tepal perianth yellow, $3.5-5 \mathrm{~mm}$ long. Anthers to $2 \mathrm{~mm}$ long. Fruit: a capsule. 


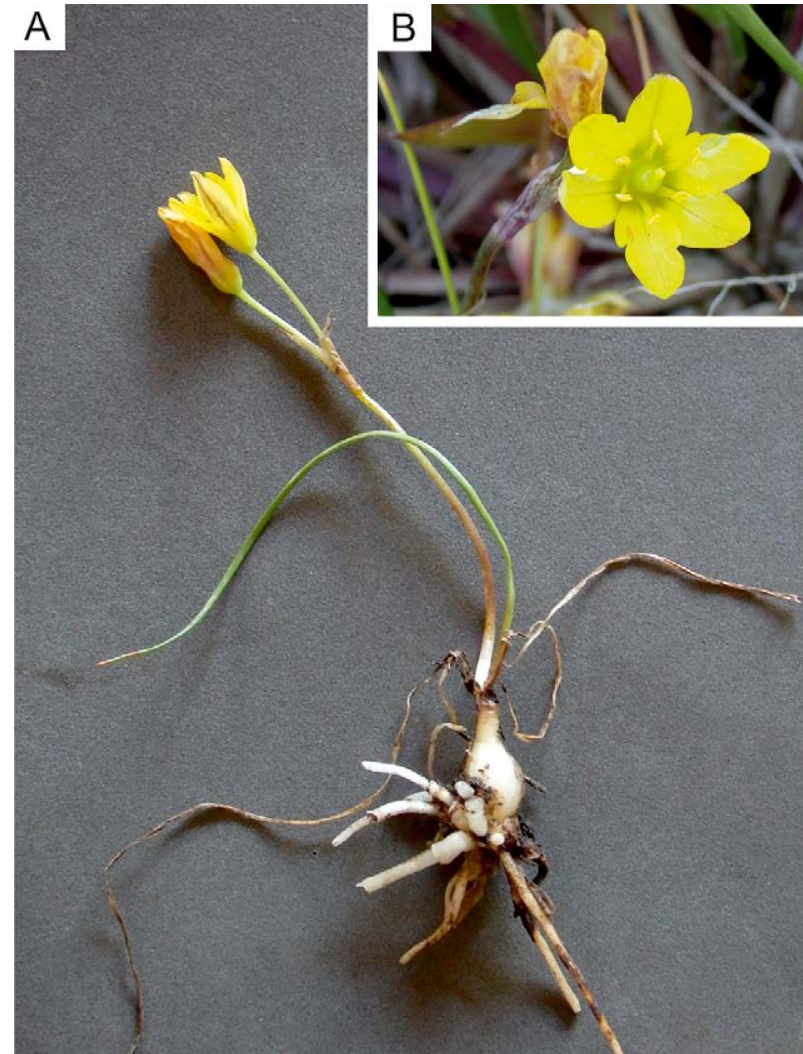

Figure 7. Nothoscordum montevidense ssp. latitepalum. A. Plant. B. Flower.

Comments. This is an infrequent species in the study area. It grows in shallow to moderately deep, damp soils on rocky and grassy slopes with other white-flowered Nothoscordum species. Cabrera (1968) reported $N$. montevidense from Buenos Aires province but not this subspecies. Hurrell (2009b) reported the existence of 3 subspecies in the Rioplatense region of northwestern Buenos Aires province. Our Paititi specimens belong to $N$. montevidensis ssp. latitepalum because they are relatively tall and present flowers with wide yellow tepals.

Order Asparagales: Family Iridaceae: Tribe Tigrideae

Genus Cypella Herb.

\section{Cypella herbertii Hooker subsp. wolffhuegeli (Hauman) Ravenna}

Figure 8

Cypella herbertii Hooker subsp. wolffhuegeli (Hauman) Ravenna

(1965): 312—Cabrera (1968): 548; Hurrell (2009a): 270; Flora Argentina (2017); Tropicos (2017).

Cypella wolffhuegeli Hauman (1909): 84.

Cypella herbertii Hooker (1826): 2637.

Cryptophyte, endemic to mountain ranges of Buenos Aires province. Vulnerable due to restricted habitat (Delucchi 2006).

Characteristics. Perennial herb. Bulbs subglobose. Peduncles erect, branched, to $1 \mathrm{~m}$ tall. Basal leaves scarce, linear in shape, parallel-veined, folded, to $35 \mathrm{~cm}$ long, $2.5 \mathrm{~cm}$ wide. Inflorescence: a cyme (rhipidium); bracts unequal, with 1-2 flowers; tepals orange-brown, filaments free, styles entire. Fruit: a capsule.

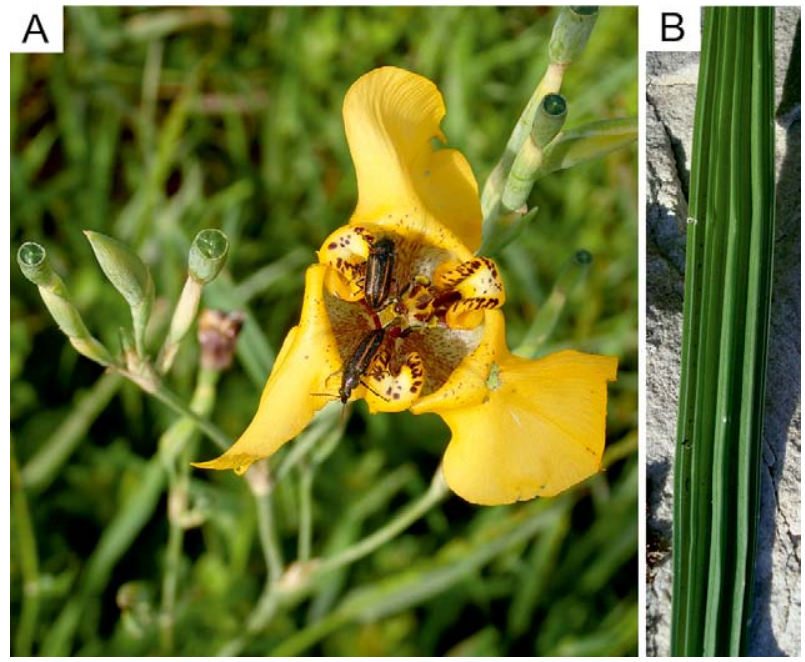

Figure 8. Cypella herbertii subsp. wolffhuegeli. A. Flower. B. Leaf detail.

Comments. This is a rare species in the study area. It grows in full sunlight conditions in rock cracks on slopes. It is very similar to $C$. herbertii ssp. herbertii, but this last species presents vivid orange tepals and united filaments.

Genus Gelasine Herb.

\section{Gelasine elongata (Graham) Ravena}

Figure 9

Gelasine elongata (Graham) Ravena (1988): 154-Flora Argentina (2017); Tropicos (2017).

Ferraria elongata Graham (1830): 173.

Gelasine azurea Herbert (1840): 3779—Cabrera (1968): 543.

Cryptophyte, native with distribution in the Southern Cone Region of South America.

Characteristics. Perennial bulbous herb, to $80 \mathrm{~cm}$ tall. Basal leaves linear, ca $25 \mathrm{~cm}$ long, $3 \mathrm{~cm}$ wide, sword-
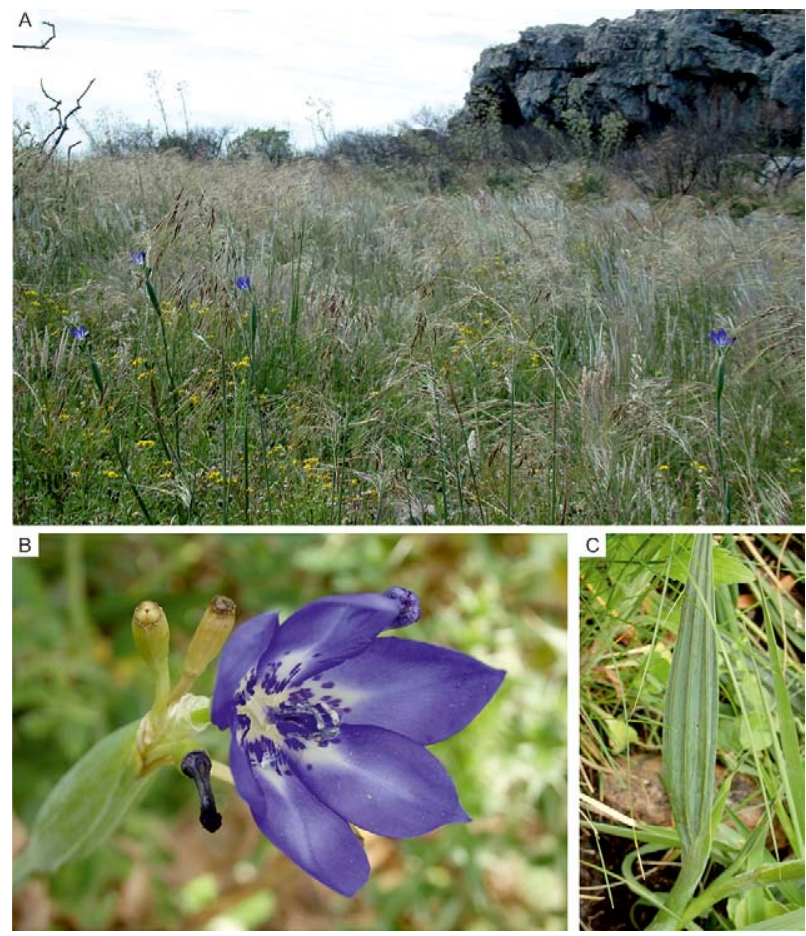

Figure 9. Gelasine elongata. A. Plants. B. Flower. C. Leaf detail. 


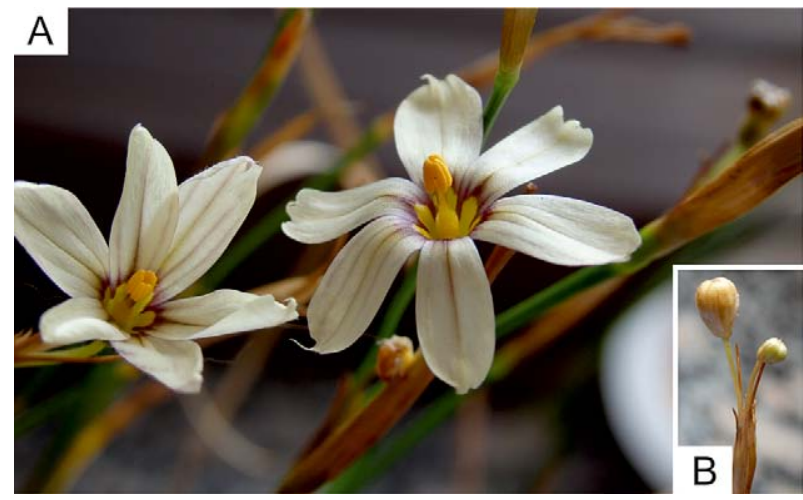

Figure 10. Sisyrinchium sp. A. Flowers. B. Fruits.

shaped, folded, parallel-veined, semi-coreaceus; superior leaves scarce. Inflorescence: a cyme with 1-3 flowers; tepals blue with dark spots at base. Fruit: a capsule.

Comments. This is an infrequent species in the study area. It grows in full sunlight conditions and deep to moderate deep soils on rocky and grassy slopes. Gelasine elongate is the only species of its genus known from Buenos Aires province, and it can be recognized by its folded leaves and blue tepals.

Order Asparagales: Family Iridaceae: Tribe Sisyrinchieae Genus Sisyrinchium L.

\section{Sisyrinchium sp.}

Figure 10

Cryptophyte, native.

Characteristics. Perennial herb, $30-50 \mathrm{~cm}$ tall. Roots fleshy. Basal leaves numerous, erect, parallel-veined, sharply folded along their midribs, 20-27 cm long. Peduncle winged, with a multiflorous cyme (rhipidium); flowers white to pale yellow, with violet nerves and yellow spots at base, surrounded by purplish tinge; tepals with emarginate-apiculate apex. Filaments entirely connate into a glabrous column; base of the filaments coverd with yellow glands. Fruit: a globose or obovate capsule.

Comments. This is a frequent species in the study area. It grows in full sunlight conditions and shallow to moderately deep soils on rocky and grassy slopes. The specimens of the Paititi Reserve have fleshy roots and their filaments are grouped forming a column. The base of this column is covered with glands so it would correspond to Sisyrinchium platense I.M. Johnst. or S. pachyrhizum Baker (Cabrera 1968, Roitman and Hurrell 2009). However, in S. platense the tepals are violet to pale blue, and in $S$. pachyrhizum they are vivid yellow with brown spots. As neither of the described species for Buenos Aires province present tepals with the colors seen in our specimens, we have not identified our material to species.

Order Asparagales: Family Orchidaceae: Tribe Chloraeeae

\section{Genus Bipinnula Commerson}

Bipinnula pennicillata (Rch. f.) Cisternas \& Salazar Figure 11

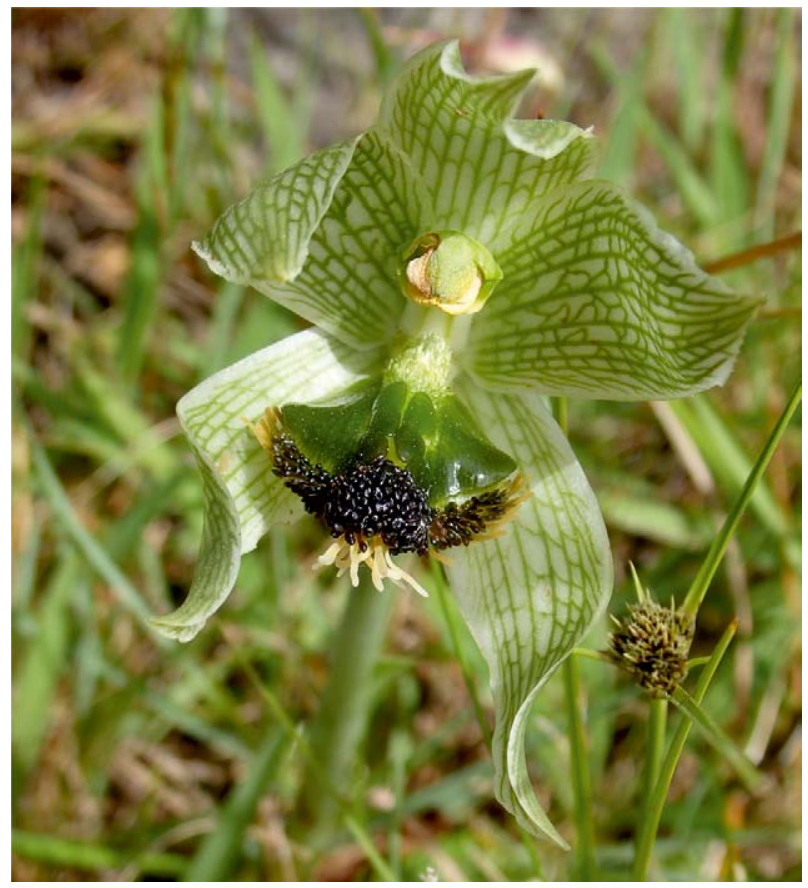

Figure 11. Bipinnula pennicillata. Flower.

Bipinnula pennicillata (Rchb. f.) Cisternas \& Salazar ex Cisternas et al. (2012): 10_Flora Argentina (2017); Tropicos (2017).

Geoblasta pennicillata (Rchb. f.) Hoehne ex Correa (1968): 71Cabrera (1968): 591; Hurrell (2009a): 348.

Chloraea pennicillata Reichenbach. f. (1878): 51.

Cryptophyte, native with distribution in the Southern Cone Region of South America. Vulnerable due to restricted habitat (Delucchi 2006).

Characteristics. Terrestrial herb, to $30 \mathrm{~cm}$ tall including inflorescence. Roots narrow, also tuberous roots. Basal leaves 3 or 4, with elliptic- to ovate-lanceolate in shape, to $10 \mathrm{~cm}$ long, larger than caulinar leaves. Inflorence 1-flowered, exceptionally 2-flowered; flower pale green, with reticulate dark green veins and an insectlike labellum with a darker, blackish-brown, apical part; labellum sessile, smooth, shiny, greenish, convex, its basal part covered by short, slender, retrorse, pale green hairs; central disk with 6 or 7 naked ridges; apex covered with short, clavate, blackish-brown and yellow appendices. Fruit: a capsule.

Comments. This is an infrequent species in the study area. It grows in full sunlight conditions in shallow soils on grassland slopes and on summits. Bipinnula pennicillata can be recognized from other Orchidaceae by its 1-flowered inflorescence and its large flowers with rhombic, succulent labellum and sepals without appendices.

Genus Chloraea Lindl.

\section{Chloraea membranacea Lindley}

Figure 12

Chloraea membranacea Lindley (1840): 401-Cabrera (1968): 588; Hurrell (2009a): 344; Flora Argentina (2017); Tropicos (2017).

Cryptophyte, native with distribution in the Southern Cone Region of South America. 
Characteristics. Terrestrial herb, $40-70 \mathrm{~cm}$ tall, including inflorescence, with folded, fleshy roots. Basal leaves 5 or 6 , elliptic in shape, to $15 \mathrm{~cm}$ long, with subacute apex. Inflorescence: a raceme; flowers greenish-white; labellum margin entire or slightly trilobed, covered by cilindrical, capitated, darker, blackish-brown to black appendices. Stamens with anthers inclined. Fruit: a capsule.

Comments. This is a rare species in the study area. It grows in damp soils, in shady places under trees. Chloraea membranacea is the only species of Chloraea known from Buenos Aires province and is distinguished from other Orchidaceae by its folded, thickened, fleshy roots, and its inclined anthers.

Order Poales: Family Bromeliaceae: Tribe Tillandsieae

Genus Tillandsia L.

\section{Tillandsia bergeri Mez}

Figure 13

Tillandsia bergeri Mez (1916): 254-Cabrera (1968): 455; Flora Argentina (2017); Tropicos (2017).

Epiphyte, native with distribution in the Southern Cone Region of South America. Vulnerable for reasons unknown (Delucchi 2006).

Characteristics. Perennial plant, to $30 \mathrm{~cm}$ tall, with branched roots, and stems covered with sheathed leaves. Leaves lanceolate, forming rosettes, covered with grey scales. Peduncles short, with bracts. Inflorescence: a spike with 6-10 flowers, 25-30 mm long; floral bracts and sepals green to pink; petals blue. Fruit: capsule.

Comments. This is an infrequent species in the study area. It grows in full sunlight conditions in cracks of rocks on summits, where it forms hanging clumps. It is the only species of the genus that has been found in the Tandilia mountain range system. It is the only lithomorphic species found in rocky outcrops and presents dense roots and leaves, multiflorous spikes and green to pink floral bracts and sepals, not reddish like T. aëranthos (Loisel) L. B. Smith.

Order Poales: Family Poaceae: Tribe Poeae

Genus Bromidium Nees \& Meyen

\section{Bromidium tandilense (Kuntze) Rúgolo}

\section{Figure 14}

Bromidium tandilense (Kuntze) Rúgolo (1982): 2002-Zuloaga et al. (2012b): 260; Flora Argentina (2017); Tropicos (2017).

Agrostis tandilensis (Kuntze) Parodi (1943): 158-Cabrera (1970): 231. Bromidium hygrometricum var. tandilense Kuntze (1898): 343.

Therophyte, native with distribution in the Southern Cone Region of South America; previously reported from Buenos Aires province but not from mountainous areas.

Characteristics: Annual herb, 5-50 cm tall. Leaves linear, parallel-veined, to $10 \mathrm{~cm}$ long, green, glabrous, with membranaceous ligule. Inflorescence: a spike-like panicle, to $8 \mathrm{~cm}$ long, with many 1-flowered spikelets, ca $3 \mathrm{~mm}$ long; glumes unequal; lemma with 4.5-6 mm long awns; palea absent. Fruit: a fusiform caryopsis.

Comments. This is an infrequent species in the study area. It grows in shallow soils and rock cracks on the sum-

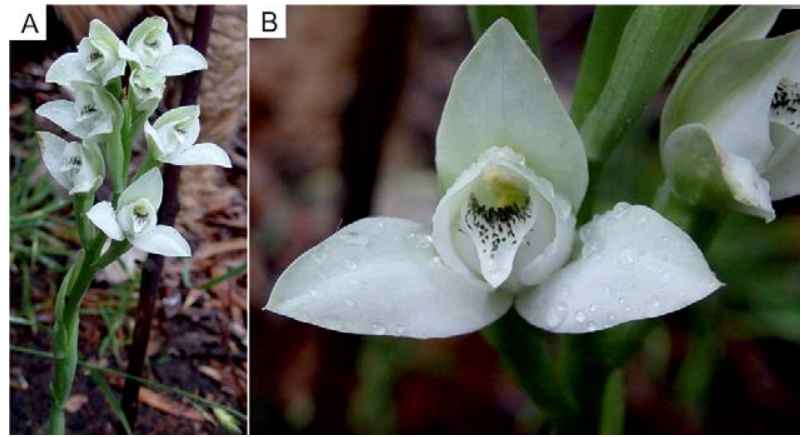

Figure 12. Chloraea membranacea. A. Inflorescence. B. Flower.
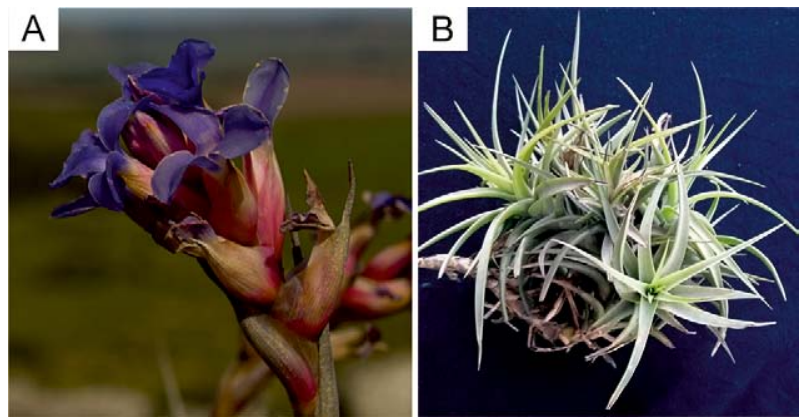

Figure 13. Tillandsia bergeri. A. Inflorescence. B. Leaf detail.

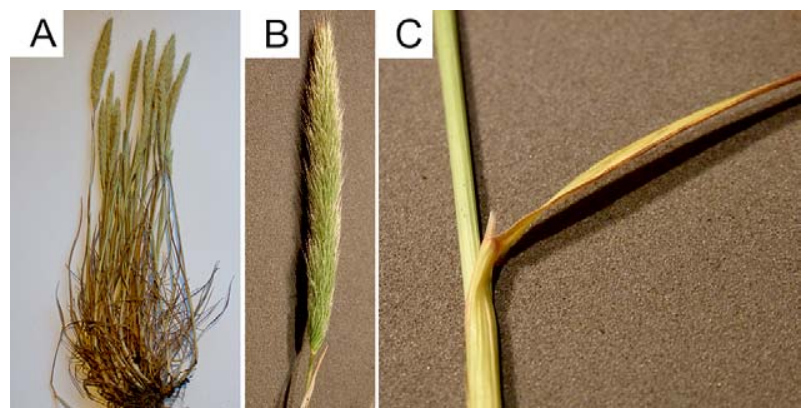

Figure 14. Bromidium tandilense. A. Plant. B. Inflorescence. C. Leaf detail.

mits. It is similar to Bromidium hygrometricum (Ness) Ness \& Meyen, which also has been found in Buenos Aires province, but $B$. hygrometricum has a glabrous lemma.

Order Poales: Family Poaceae: Tribe Danthonieae

Genus Danthonia DC

\section{Danthonia montevidensis Hack. \& Arechav.}

Figure 15

Danthonia montevidensis Hack. \& Arechav. (1896): 369-Zuloaga et al. (2012a): 229; Flora Argentina (2017); Tropicos (2017).

Hemicryptophyte, native with distribution in the Southern Cone Region of South America; previously known from Buenos Aires province but not from mountainous areas. Available information is inadequate to make a direct or indirect assessment of its risk of extinction (Data Deficient) (Delucchi 2006).

Characteristics: Perennial, caespitose species herbs, $15-60 \mathrm{~cm}$ tall. Young leaves are folded to rolled. Leaves parallel-veined, green, glabrous, to $30 \mathrm{~cm}$ long. Inflores- 


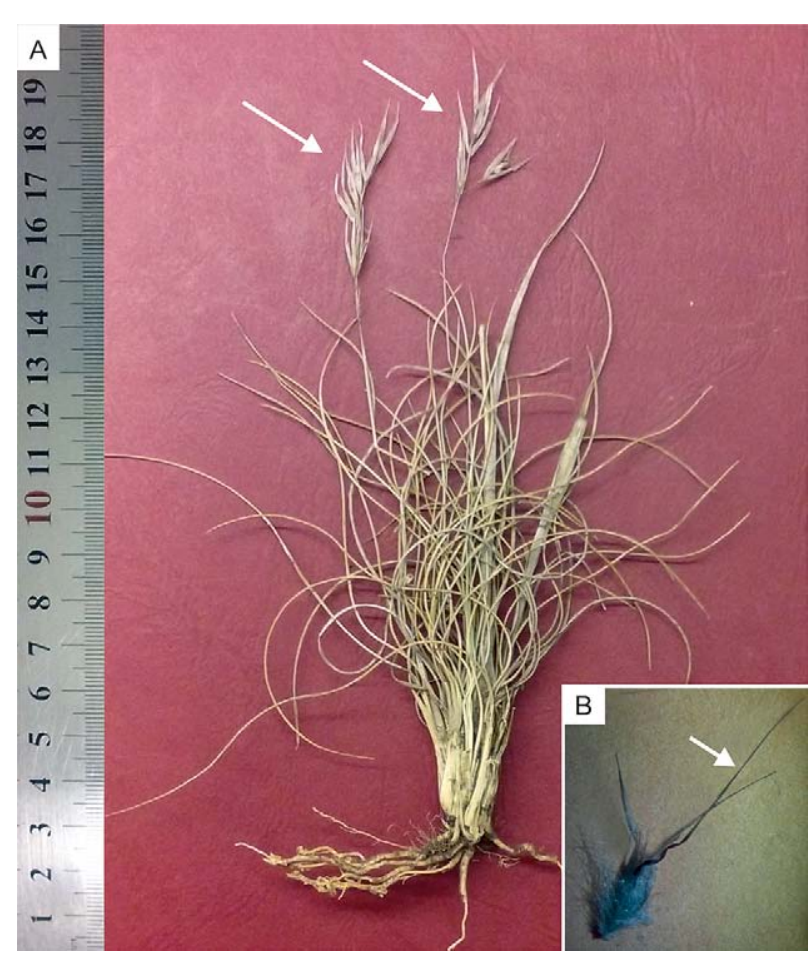

Figure 15. Danthonia montevidensis. A. Plant with two inflorescences (arrows). B. Flower and awn detail (arrow).

cence: a loose pubescence panicle, to $15 \mathrm{~cm}$ long, with multiflorous lanceolate spikelets. Glumes 16-30 mm long, outgrowing flower sets. Spikelets with 5-10 flowers; lemma 2.5-4 mm long, ovate-lanceolate, with bifid apex. Central twisted awn brown or colorless, 15-23 cm long, inserted at bifurcation point of lemma. Fruit: an obovate caryopsis.

Comments. This is a frequent species in the study area. It grows in shallow to moderately deep soils on slopes, in grasslands, and around the pond. It is similar to Danthonia cirrata Hack. \& Arechav., which also has been found in the Paititi Reserve, but the lemma of the latter species has the lateral lobes longer than the undivided part.

Order Poales: Family Poaceae: Tribe Meliceae

Genus Melica L.

\section{Melica rigida Cavanilles}

Figure 16

Melica rigida Cavanilles (1799): 47—Zuloaga et al. (2012b): 94; Flora Argentina (2017); Tropicos (2017).

Melica aurantiaca Desr. ex Lam. var. rigida (Cav.) Papp (1928): $352-$ Zuloaga et al. (2012b): 94.

Melica brasiliana auct. non Arduino (1764): 17.

Melica papilionacea auct. non. L. (1767): 31.

Cryptophyte, native with distribution in the Southern Cone Region of South America.

Characteristics. Perennial, caespitose species, 20-80 $\mathrm{cm}$ tall, with short rhizomes and erect culms. Young leaves are folded to rolled. Leaves parallel-veined, green, glabrous to hispid, to $9 \mathrm{~cm}$ long. Inflorescence: a linear, loose panicle, white with purplish tinge, compressed, multiflorous spikelets, to $11 \mathrm{~mm}$ long; glumes unequal;
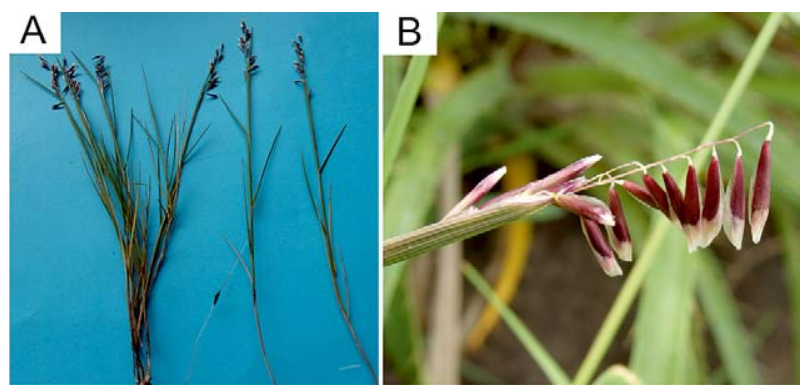

Figure 16. Melica rigida. A. Plants. B. Spikelet detail.

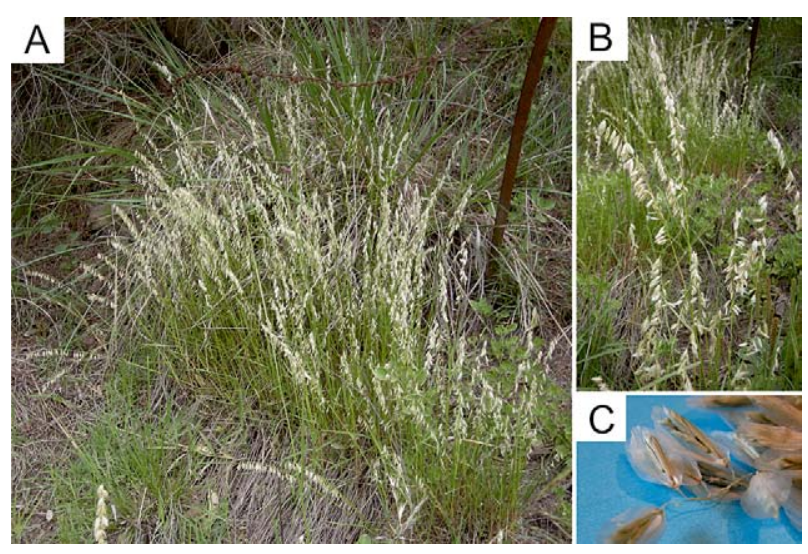

Figure 17. Melica parodiana. A. Plant. B. Inflorescence detail. C. Spikelet detail.

lemma papery with retrorse hairs; palea with glabrous lower surface. Fruit: an elliptic caryopsis.

Comments. This is a frequent species in the study area. It grows in shallow to moderately deep soils and in rock cracks on the summits and on rocky or grassy slopes. It is similar to M. brasiliana Ard. However, the latter species presents 11-17 mm long spikelets, which are longer than in M. rigida (Torres 1980, Zuloaga 2012b).

\section{Melica parodiana Torres}

Figure 17

Melica parodiana Torres (1968): 202_Cabrera (1970): 305; Zuloaga et al. (2012b): 94; Tropicos (2017).

Cryptophyte, native with distribution in the Southern Cone Region of South America. Vulnerable, reasons unknown (Delucchi 2006).

Characteristics. Perennial, caespitose species, with erect to slightly stretched culms, up to 30 to $50 \mathrm{~cm}$ tall, with roots on basal nodes. Leaves parallel-veined, folded to convolute, green, pubescent, up to $15 \mathrm{~cm}$ long, with retrorse hairs and membranous ligule. Inflorescence: a linear, loose panicle with white, compressed, multiflorous spikelets; glumes unequal; lemma papery with retrorse hairs and palea with glabrous lower surface. Fruit: an elliptic caryopsis.

Comments. This is a frequent species in the study area. It grows in shallow to moderately deep soils and in rock cracks on summits and on rocky and grassy slopes. It is similar to M. rigida, which has also been found in the Paititi Reserve, but $M$. rigida has glabrous abaxial leaf surface. 


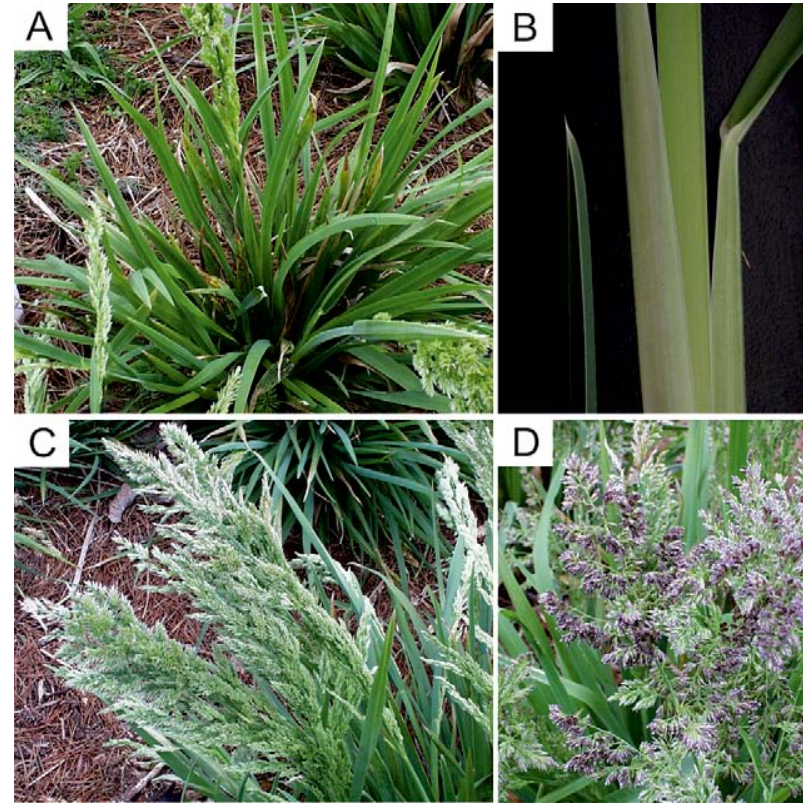

Figure 18. Poa iridifolia. A. Plant. B. Leaf detail. C. Female inflorescence. D. Male inflorescence.

Order Poales: Family Poaceae: Tribe Poeae

Genus Poa L.

\section{Poa iridifolia Hauman}

\section{Figure 18}

Poa iridifolia Hauman (1917): 407-Cabrera (1970): 115; Zuloaga et al. (2012b): 311; Flora Argentina (2017); Tropicos (2017) Poa lanigera Nees var. tandilensis Hackel (1911): 152.

Cryptophytes, endemic to mountain ranges of Buenos Aires province. Vulnerable for reasons unknown (Delucchi 2006).

Characteristics. Dioecious, perennial, caespitose species, to $1 \mathrm{~m}$ tall, with erect, 1 - or 2-node culms. Leaves parallel-veined, conduplicate, coriaceous, glaucous to glaucous-green, with rough margins and midribs. Basal innovations flabellate, leaf-sheaths compressed. Leaves plane, with a hooded apex, to $50 \mathrm{~cm}$ long; ligule membranous. Inflorescence: a compact panicle, to $20 \mathrm{~cm}$ long; female and male florets similar, multiflorous, pubescent, 3.5-6 mm long. Fruit: a fusiform caryopsis.

Comments. This is an infrequent species in the study area. It grows in shallow soils and in rock cracks on slopes and summits. It can be differentiated from other Poa species of the area by its caespitose, glaucouse foliage and by its conduplicate leaves with flabellate basal innovations, as in several Iris species.

\section{Superorder Rosanae}

Order Fabales: Family Fabaceae: Tribe Mimoseae

Genus Mimosa L.

\section{Mimosa rocae Lorentz \& Niederlein}

Figure 19

Mimosa rocae Lorentz \& Niederlein (1881): 213 - Cabrera (1967): 143;

Flora Argentina (2017); Tropicos (2017).

Chamaephytes; native with distribution in the Southern Cone Region of South America. Vulnerable, reasons unknown (Delucchi 2006).

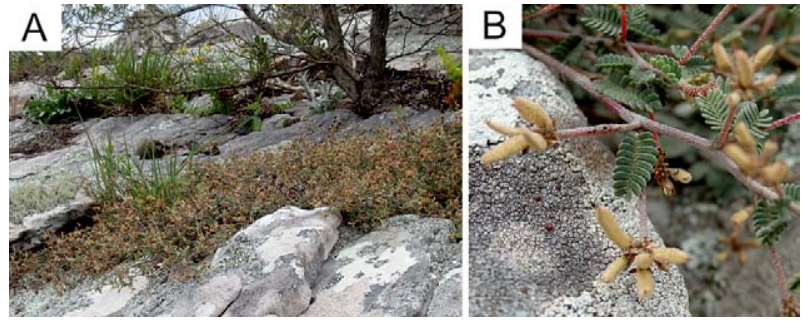

Figure 19. Mimosa rocae. A. Plant. B. Detail of stems, leaves and legumes.

Characteristics. Small shrubs covered with grey scales and with many creeping reddish branches. Leaves bipinnate, $0.3-1.5 \mathrm{~cm}$ long, with 1 pair of pinnae; leaflets imbricated, grey, 1-4 mm long, with stellate hairs. Inflorescence dense and globose, to $2 \mathrm{~cm}$ long, with yellow actinomorphic flowers. Fruits tomentose, with pale brown loments that break apart at 1-4 constrictions.

Comments. This is an infrequent species in the study area. It grows in full sunlight conditions in cracks of rocks on the summits. In the mountains of the area, $M$. tandilensis Speg. can also be found but differs from $M$. rocae by presenting prickles on the stems and purplish pink flowers.

Order Fabales: Family Fabaceae: Tribe Vicieae

Genus Lathyrus L.

\section{Lathyrus hookeri Don}

Figure 20

Lathyrus hookeri Don (1832): 332 - Cabrera (1967): 616; Flora Argentina (2017).

Lathyrus hookeri Don f. albiflora (O. Kuntze) Burkart, ex: Cabrera (1963): 616.

Lathyrus sessilifolius Hooker \& Arnott (1831): 20.

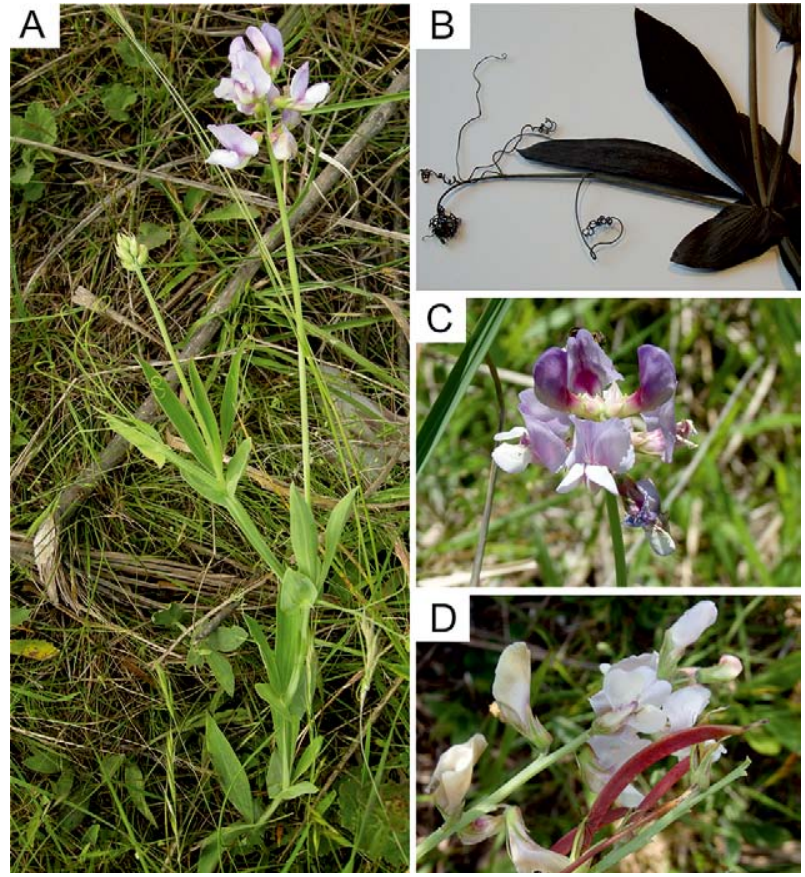

Figure 20. Lathyrus hookeri. A. Plant. B. Detail of leaves that have turn black after being dried. C. Specimen with white keel and violet standard and wings. D. Specimen with white petals. 
Cryptophytes; native with distribution in the Southern Cone Region of South America.

Characteristics. Perennial twining herb that turns black when it dries. Rhizomes and decumbent tetragone stems with 2 wings in upper stems. Leaves sessile or subsessile, pinnately compound, with 2 oval, slightly glaucous, mucronate leaflets with notable veins, 1-7 terminal tendrils, and wide sagittate stipules. Inflorescence: a raceme with 5-10 flowers of lilac to violet petals. Fruit: a linear and erect legume, which turns black at maturity.

Comments. This is a frequent species in the study area. It grows in intermediate to full sunlight, on slopes with moderately deep, damp soils. Many species of the genus Lathyrus thrive in the mountain ranges of this region. Lathyrus hookeri can be recognized by the presence of very short petioles (or even sessile leaves) and by its fruit that turns black when dried. In the Paititi Reserve, specimens with violet petals were found, which is typical, and specimens with white petals were also found that, according to Cabrera (1967), would correspond to L. hookeri Don f. albiflora (O. Kuntze) Burkart. Nowadays, L. hookeri f. albiflora is synonym of L. hookeri Don (Flora Argentina 2017). Besides the white flower specimens, plants with white keel and violet standard and wings were also found. This would be the first record of this variant of $L$. hookeri.

\section{Lathyrus pusillus Elliot}

Figure 21

Lathyrus pusillus Elliot (1823): 223-Flora Argentina (2017); Tropicos (2017).

Lathyrus crassipes Gillies, ex Hook. \& Arn. (1830): 198 - Cabrera (1967): 609.

Therophytes, native with wide distribution in the American continent; previously recorded from Buenos Aires province but not from mountainous areas.

Characteristics. Annual twining herb, to $50 \mathrm{~cm}$ tall. A glabrous plant with short-winged stems. Leaves pinnately compond, with 2 linear leaflets and a terminal tendril, split into $1-3$ segments; petiole $0.5-1.5 \mathrm{~cm}$ long, stipules sagittate. Inflorescence: racemes, with 1-3 flowers, with glabrous sepals and white to blue petals. Fruit: a linear and erect legume, turning brown at maturity.

Comments. This is an infrequent species in the study area. It grows in intermediate to full sunlight on slopes with moderately deep, moist soils. Lathyrus pusillus can be recognized from the many other Lathyrus species in the region by the presence of sagittate stipules and brown legumes that do not turn black when dry.

\section{Genus Vicia L.}

\section{Vicia linearifolia Hooker \& Arnott}

Figure 22

Vicia linearifolia Hooker \& Arnott (1830): 20 - Cabrera (1967): 588; Flora Argentina (2017).

Therophytes, native with distribution in the Southern Cone Region of South America; previously recorded from Buenos Aires province but not from mountainous areas.

Characteristics. Annual, delicate, twining herb,

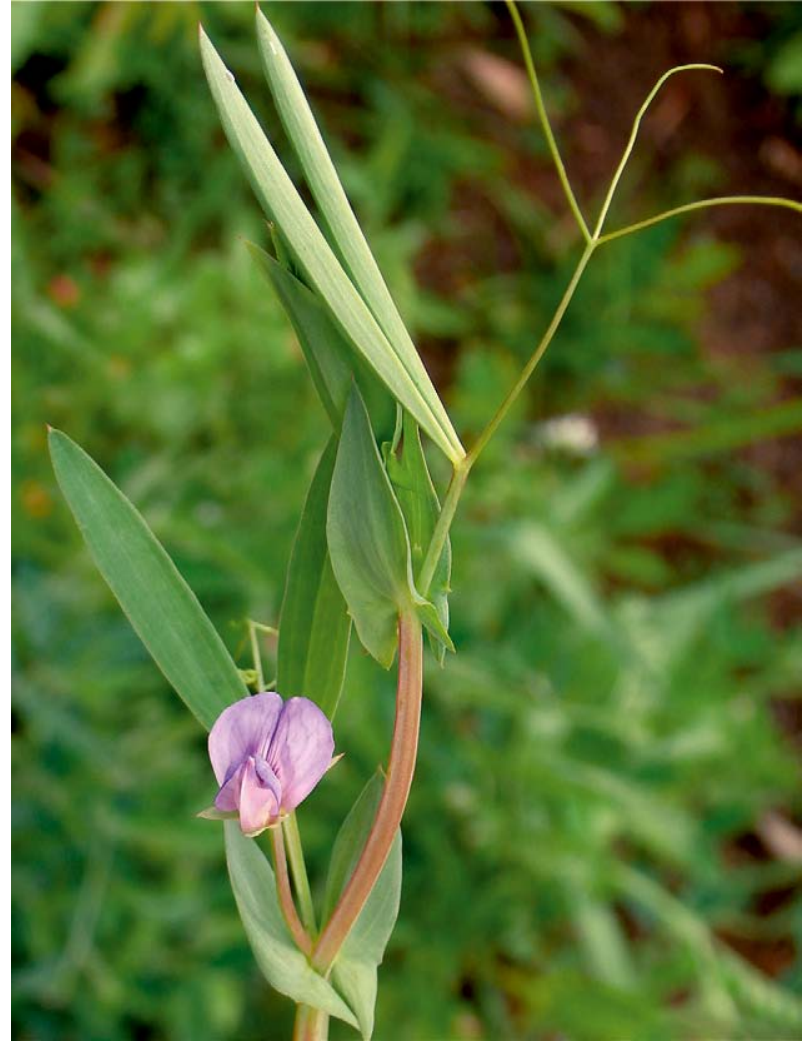

Figure 21. Lathyrus pusillus. Detail of flower and leaves.
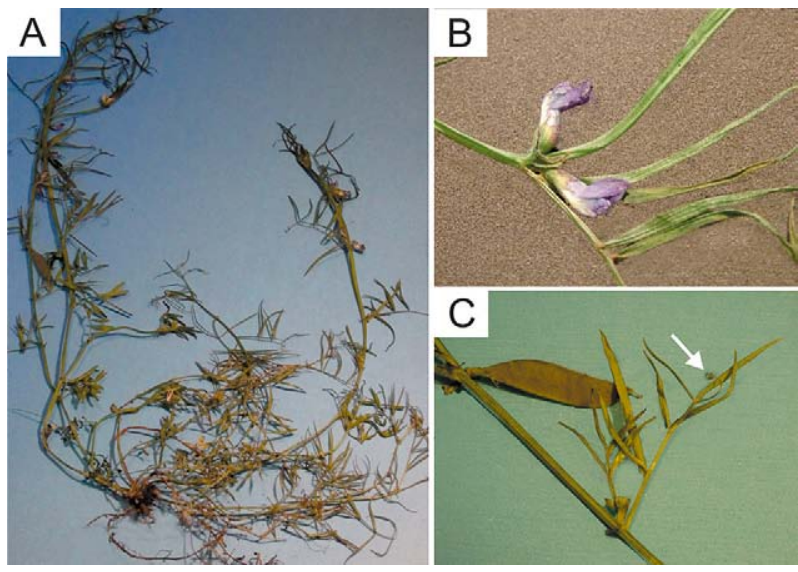

Figure 22. Vicia linearifolia. A. plant. B. detail of the flowers. C. detail of legume, leaf and simple tendril (arrow).

20-40 cm tall, glabrous, with narrow, angular stems. Leaves pubescent, pinnately compound, with 3-5 linear to filiform pairs of leaflets, $0.2-3 \mathrm{~mm}$ wide; with a terminal, simple tendril, split into 1-3 segments; petiole short or even absent; stipules semi-sagittate, with a spur. Flowers solitary in axils of stems, exceptionally in a terminal, 2-flowered cyme; corollas pale blue to blue. Fruit: an erect or pendent, pubescent, brown legume.

Comments. This is an infrequent species in the study area. It grows in shady sites under trees in damp soils on slopes. It differs from other Vicia species by the presence of solitary flowers (exceptionally 2 ) that are up to $4 \mathrm{~mm}$ long.

\section{Vicia setifolia Kunth var. bonariensis Burkart}

Figure 23

Vicia setifolia Kunth var. bonariensis Burkart (1966): 595-Cabrera 


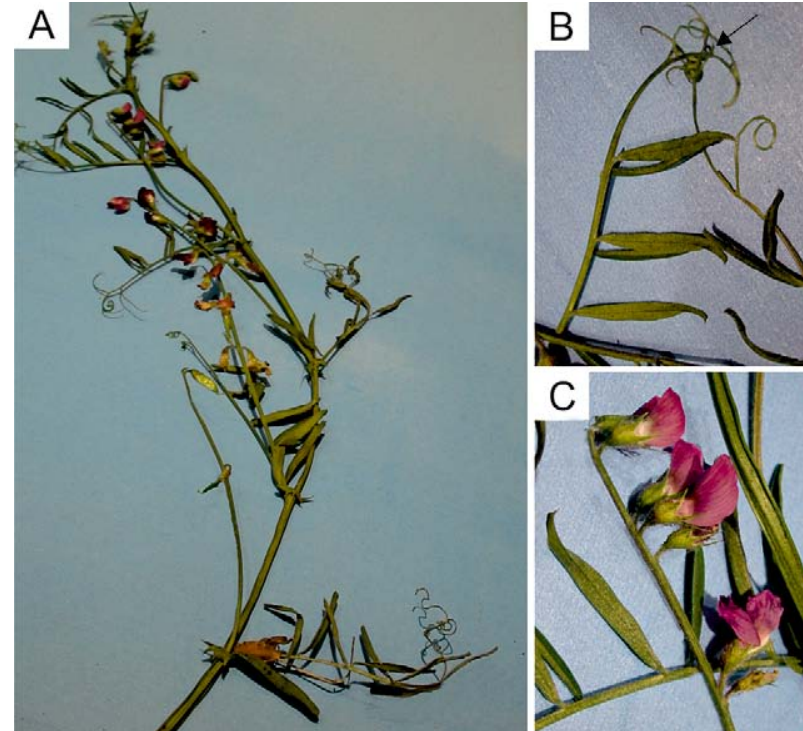

Figure 23. Vicia setifolia var. bonariensis. A. Plant. B. Detail of leaf and split tendril (arrow). C. detail of the flowers.

(1967): 595; Flora Argentina (2017); Tropicos (2017). Vicia setifolia Kunth (1823): 500-501.

Cryptophytes, endemic to mountain ranges of Buenos Aires province. Vulnerable, reasons unknown (Delucchi 2006).

Characteristics. Perennial rhizomatous twining herb. Stems pubescent, ascendent, $40-80 \mathrm{~cm}$ high, angulate to bi-winged. Leaves pubescent, pinnately compound, with 3-5 linear-lanceolate to oblong pairs of leaflets, with a terminal tendril, split into 1-3 segments; petiole short; stipules semi-sagittate, with acute apex and a spur. Inflorescence: a raceme with 4-8 flowers, with blue-violet corolla and pubescent, dentate calyx. Fruit: a compressed linear legume with a recurved apex.

Comments. This is an infrequent species in the study area. It grows in intermediate and full sunlight on slopes and in grasslands in moderately deep, moist soils. This perennial species differs from other Vicia species by the presence of peduncle racemes with flowers smaller than $1.4 \mathrm{~cm}$ long, and pubescent leaves with 3-5 linearlanceolate to oblong pairs of leaflets.

Order Fabales: Family Polygalaceae: Tribe Polygaleae

Genus Polygala L.

\section{Polygala australis A.W. Bennett}

Figure 24A, B

Polygala australis A.W. Bennett (1879): 203-Cabrera (1965a): 64; Flora Argentina (2017); Tropicos (2017).

Polygala pamparum Spegazzini (1901b): 240.

Therophytes, native with distribution in the Southern Cone Region of South America.

Characteristics. Annual or biennial herb, with erect or prostrate leafy stems, $5-15 \mathrm{~cm}$ tall. Leaves simple, linear in shape, ca 6-8 $\mathrm{cm}$ long and $1-1.7 \mathrm{~mm}$ wide. Inflorescence: a spike-like raceme, to $2.5 \mathrm{~cm}$ long, with zygomorphic, perfect flowers, ca $2 \mathrm{~mm}$ long, with white petals. Fruit: an orbicular capsule that contains seeds with a ring of hairs.
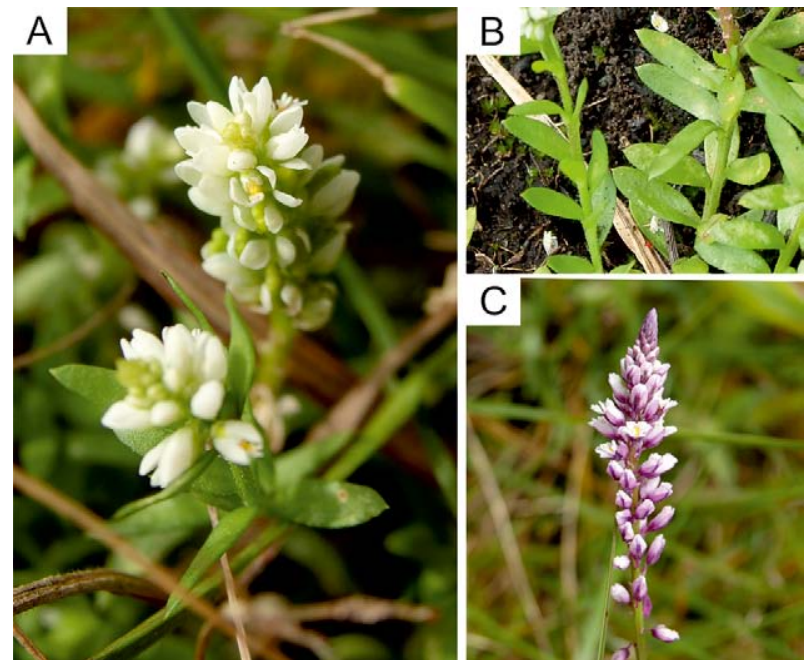

Figure 24. A. Detail of Polygala australis flowers. B. Detail of P. australis leaves. C. Detail of $P$. linoides flowers.

Comments. This is a frequent species in the study area. It grows in shallow, damp soils on grassland slopes and on the summits. Polygala australis shares habitat with $P$. linoides Poir., which, however, is a perennial herb with lilac flowers (Fig. 24C) and presents seeds without a ring of hairs.

Order Malvales: Family Malvaceae: Tribe Hibisceae

Genus Pavonia Cav.

\section{Pavonia cymbalaria A. St.-Hil. \& Naudin}

Figure 25

Pavonia cymbalaria A. St.-Hil. \& Naudin (1842): 42—Cabrera (1965a): 176; Flora Argentina (2017); Tropicos (2017).

Chamaephytes, native with distribution in the Southern Cone Region of South America.

Characteristics. Subshrub with many creeping branches, to $60 \mathrm{~cm}$ long, and densely pubescent ascending stems. Leaves with stipulates; ovate to triangular, margins crenate, glaucous, covered with short hairs. Flowers solitary; epicalyx with oval bracts; corolla with pink-lilaceous petals; nerves and basal stained red to violet. Fruit: a schizocarp, with reticulated mericarps.

Comments. This is a frequent species in the study area. It grows in shallow to moderately deep soils on slopes, including grassy slopes, and on summits. In the mountain ranges also grows Pavonia glechomoides A.St. Hil., which differs from P. cymbalaria by the presence of long hair pubescence, suborbiculate leaves, thin epicalyx bracts, and white or pinkish petals with the basal stain purple.

Order Myrtales: Family Myrtaceae: Tribe Myrteae

Genus Psidium L.

\section{Psidium salutare (H.B.K.) O. Berg var. mucronatum (Cambess.) Landrum}

Figure 26

Psidium salutare (H. B. K.) O. Berg var. mucronatum (Cambess.) Landrum (2003): 1463 - Flora Argentina (2017); Tropicos (2017).

Psidium luridum (Sprengel) Burret (1941): 484—Cabrera (1965a): 305. 

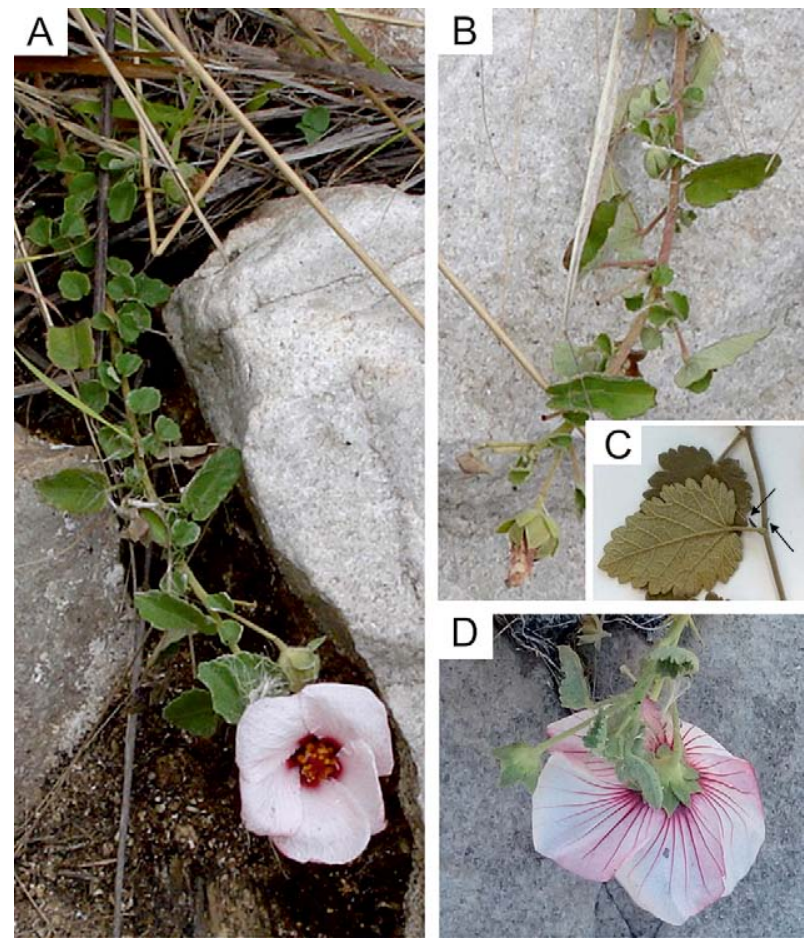

Figure 25. Pavonia cymbalaria. A. Flower. B. Stem and leaves. C. Detail of the leaves and stipulates (arrows). D. Detail of the back of the petals.

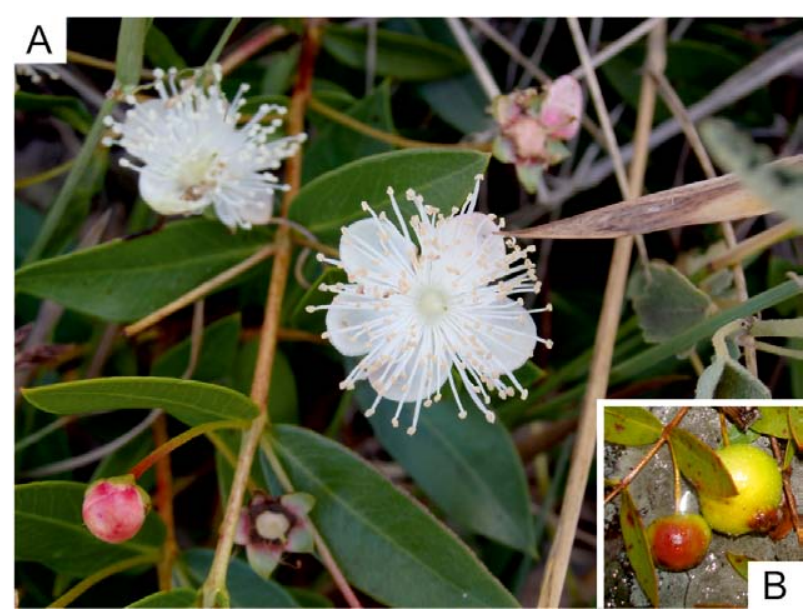

Figure 26. Psidium salutare var. mucronatum. A. Stems and flowers. B. Immature fruits.

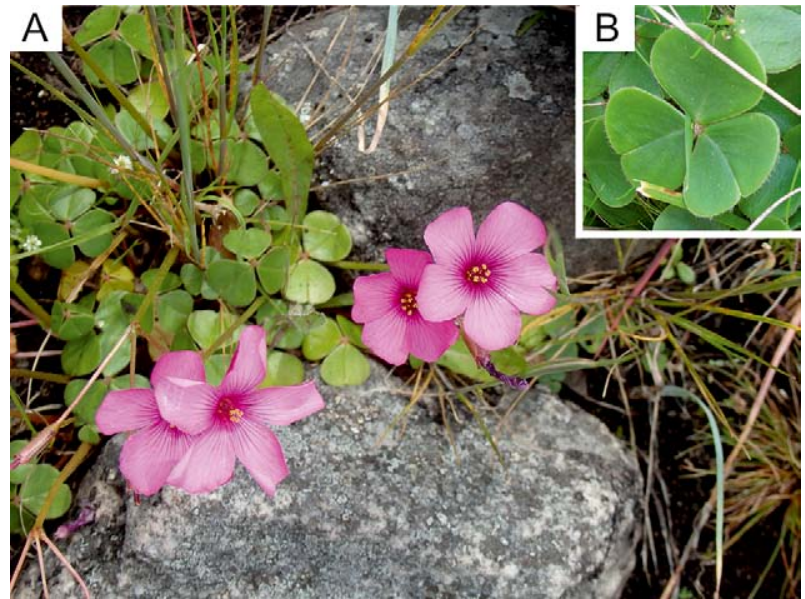

Figure 27. Oxalis brasiliensis. A. Plant. B. Leaflet detail.

Myrtus mucronata Cambessèdes ex A. St. Hilaire (1829): 294.

Myrtus salutaris Kunth ex H.B.K (1823): 132.

Cryptophytes, native with distribution in the Southern Cone Region of South America.

Characteristics. Aromatic, dwarf shrub, to $10-50 \mathrm{~cm}$ tall, with rhizomes and many creeping branches. Leaves opposite, elliptic, with apex acuminate. Flowers solitary, axillary, with 5 white petals, 5 sepals, numerous stamens; pedicels shorter than leaves. Fruit: a globose, green to red berry with persistent calyx.

Comments. This is a frequent species in the study area. It grows in shallow soils and in rock cracks on summits and in bare and grassy slopes. In mountain ranges it grows as a small shrub, to $20 \mathrm{~cm}$ tall. It is the only species of Psidium occurring in Buenos Aires province. It is recognized from other species of Mirtaceae by its creeping form and the number of sepals.

Order Oxalidales: Family Oxalidaceae

Genus Oxalis L. Subsection Austro-Americanae

Oxalis brasiliensis G. Lodd, C. Lood \& W. Lood.

Figure 27

Oxalis brasiliensis G. Lodd, C. Lood \& W. Lood ex Hildebrand (1884): 1962 - Lourteig (2000): 201; Flora Argentina (2017); Tropicos (2017).

Oxalis macachin Arechav. (1898) ex Arechavaleta (1900): 219Cabrera (1965a): 6.
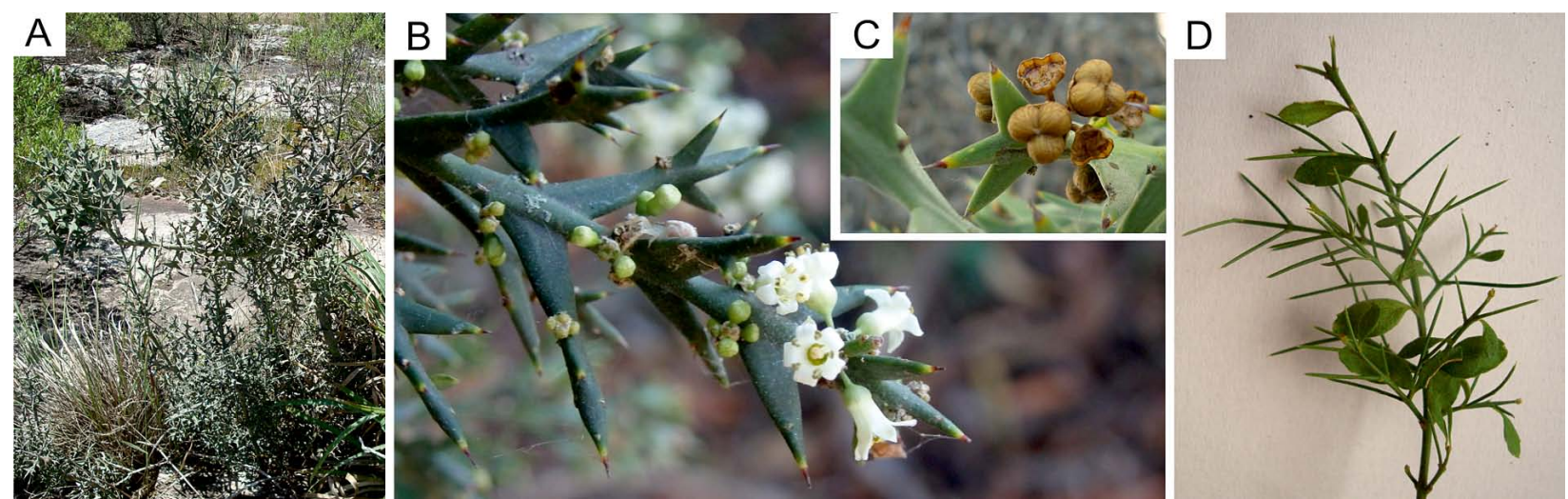

Figure 28. A. Plant of Colletia paradoxa. B. Stems and flowers of C. paradoxa. C. Fruits of C. paradoxa. D. Stems of Colletia spinosissima. 
Cryptophytes, native with wide distribution in the American continent.

Characteristics. Perennial herb with pivoting and fleshy roots. Bulb globose, covered with scales from the leaf sheaths. Leaves long, petiolated, trifoliate, pubescent and arranged in a basal rosette. Leaflets semi-fleshy, obovate shape and with subtle emarginated apex. Inflorescence: an umbel, with 3-5 actinomorphic flowers with five pink to purple petals and five sepals. Fruit: a cylindrical capsule.

Comments. This is a very frequent species in the study area. It grows in grassy slopes, cracks, and rocky outcrops on the slopes and on the summit. It differs from other Oxalis that have also been found in mountain ranges, by its simple bulb, leaflet with subtle emarginated apex and inflorescence with at least 3 pinkish flowers.

Order Rosales: Family Rhamnaceae: Tribe Colletiae

\section{Genus Colletia Commers}

\section{Colletia paradoxa (Spreng.) Escalante}

Figure 28A-C

Colletia paradoxa (Spreng.) Escalante (1946): 219—Cabrera (1965a):

163; Tortosa (1995): 4; Flora Argentina (2017); Tropicos (2017).

Condalia paradoxa Sprengel (1825a): 825.

Colletia cruciata Gillies \& Hooker (1829): 152.

Phanerophytes; native with wide distribution in the Southern Cone Region of South America. Least Concern (Delucchi 2006).

Characteristics. Rhizomatous, glaucous shrub, to 3 $\mathrm{m}$ tall. Spines triangular, compressed, decussate, without marked leaf nodes. Leaves ovate-lanceolate, to 7 $\mathrm{mm}$ long, promptly deciduous. Inflorescence: fascicle with 2-9 flowers. Flowers white, ca $1 \mathrm{~cm}$ long; sepals oblong-lanceolate, reflexes; corolla absent. Fruit: 3-locular capsule.

Comments. This is a very frequent species in the study area. It grows in intermediate and full sunlight conditions on grasslands and on slopes in moderately deep, moist soils. It also grows under the trees and in cracks of the slopes and summit. This species could be confused with other Rhamnaceae that are frequently found in the mountain ranges of Buenos Aires province: Colletia spinosissima J. F. Gmel. and Discaria americana Gillies \& Hook. These species differ from C. paradoxa in vegetative morphologic characters. Colletia spinosissima has dark yellowish-green branches and cylindrical spines (Fig. 28D), while D. americana has leaf nodes with a transversal mark (Tortosa 1995).

Superorder Caryophyllanae

Order Caryophyllales: Family Cactaceae: Tribe

Trichocereeae

Genus Gymnocalycium Pfeiff.

\section{Gymnocalycium gibbosum (Haw.) Pfeiffer}

\section{Figure 29}

Gymnocalycium gibbosum (Haw.) Pfeiffer ex Mittler (1844): 124Cabrera (1965b): 285; Flora Argentina (2017); Tropicos (2017). Cactus gibbosus Haworth (1812): 173.
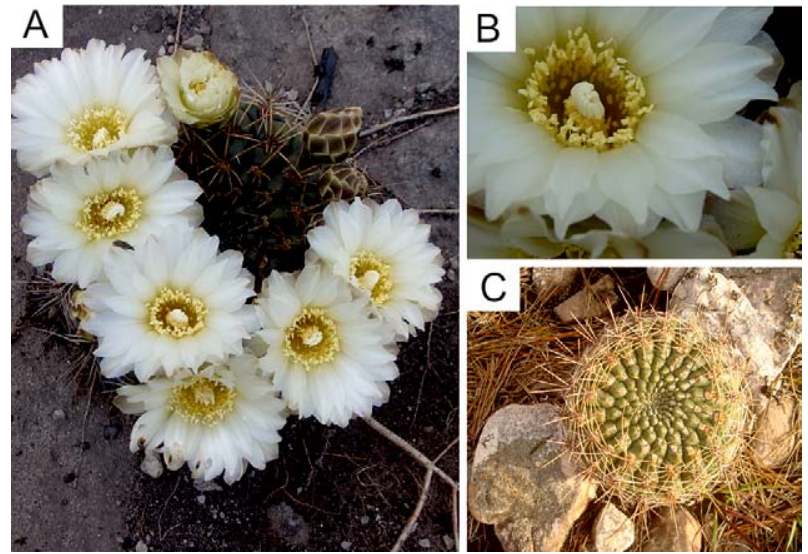

Figure 29. Gymnocalycium gibbosum. A. Plant at blooming. B. Flower. C. Plant in vegetative stage.

Gymnocalycium platense (Speg.) Britton \& Rose (1922): 163 - Cabrera (1965b): 286.

Gymnocalycium brachypetalum Spegazzini (1925): 139-Cabrera (1965b): 286.

Chamaephytes; native with wide distribution in the Southern Cone Region of South America. Vulnerable, reasons unknown (Delucchi 2006).

Characteristics. Perennial, glaucous to green herb with greatly enlarged, globose stem, to $20 \mathrm{~cm}$ long, 10 $\mathrm{cm}$ wide, and 12-16 lobed ribs. Areoles with 7-15 rigid spines. Flowers located in the superior areoles, $6 \mathrm{~cm}$ wide. Perianth white; external scales green, with ovate shape and acute apex. Fruit: fusiform or piriform shape.

Comments. This is a frequent species in the study area. It grows in full sunlight conditions in cracks on the slopes. It differs from other Cactaceae from the study area by the color of the flowers; Wigginsia and Parodia species have yellow flowers, while G. gibbosum has white flowers.

Superorder Asteranae

Order Apiales: Family Apiaceae: Tribe Saniculeae

Genus Eryngium L.

\section{Eryngium regnellii Malme}

Figure 30

Eryngium regnellii Malme (1904): 9—Calviño \& Martínez (2007): 71; Flora Argentina (2017); Tropicos (2017).

Hemicryptophytes, native with wide distribution in the Southern Cone Region of South America.

Characteristics. Perennial herbs with rhizomes and erect branches, to $2 \mathrm{~m}$ tall. Leaves glabrous, membranous; basal leaves flaccid, linear, with parallel veins and appressed, solitary bristles in the margin (frequently 2 at the base), to $1 \mathrm{~m}$ long and $15 \mathrm{~mm}$ wide, larger than the caulinar leaves. Inflorescence: a dichotomous cyme, with ovoid, greenish-white capitulums $(1.2 \times 1 \mathrm{~cm})$. Fruit: a schizocarp composed of 2 mericarps with lateral scales and vesicles on the upper lower surface, rare or absent in the base.

Comments. This is a frequent species in the study area. It grows in full sunlight conditions, in moderately deep, damp soils and in cracks on the slopes. Up to 2007, E. regnellii was confused with E. stenophyllum Urb. 


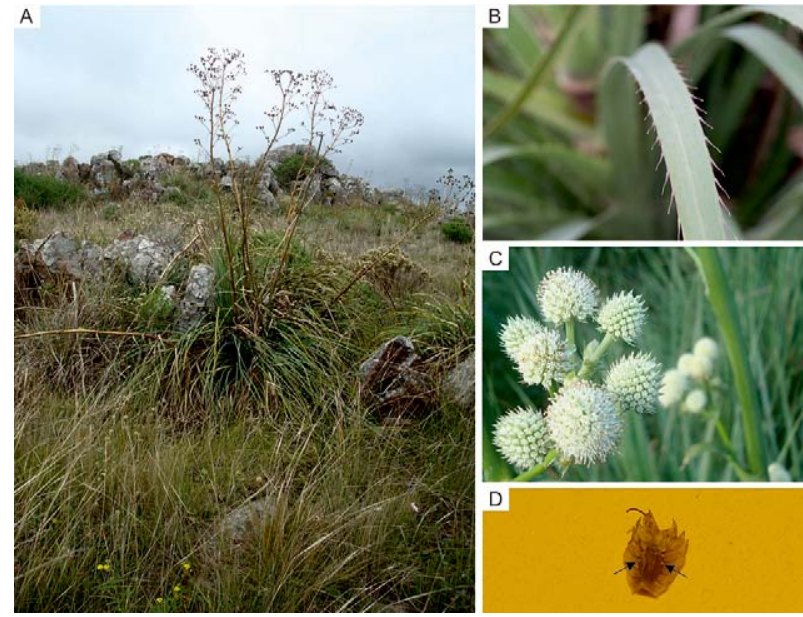

Figure 30. Eryngium regnellii. A. Plant. B. Detail of the presence of solitary bristles in the leaf margin. C. Lateral inflorescences. D. Vesicles on the upper back of the mericarp (arrow).

(Calviño and Martínez 2007), a species reported from Buenos Aires province. Eryngium stenophyllum differs from $E$. regnellii in leaf and mericarp characteristics. It presents coriaceous leaves with 1-3 bristles that are up to $15 \mathrm{~mm}$ long at the margin, and mericarps with lateral free scales and a naked lower surface.

Order Asterales: Family Asteraceae: Tribe Astereae

Genus Baccharis L.

\section{Baccharis dracunculifolia DC ssp. tandilensis (Speg.) Giuliano}

Figure 31

Baccharis dracunculifolia DC ssp. tandilensis (Speg.) Giuliano ex Zuloaga et al. (2014a): 71-Flora Argentina (2017).

Baccharis tandilensis Spegazzini (1901a): 26-Cabrera (1963): 122. Baccharis dracunculifolia De Candolle (1836): 421.

Phanerophytes; endemic to mountain ranges of Buenos Aires province. Critically Endangered, reasons unknown (Delucchi 2006).

Characteristics. Dioecious species. Globose shrub, 1-5 $\mathrm{m}$ tall. Young branches pubescent with alternated leaves. Leaves: obovate shape, length-width ratio between 1.8:1 and 3.6:1. Capitulum: sessile or subsessile, with yellow florets and white pappus. Male capitulum with tubular disc florets, up $3 \mathrm{~mm}$ long; female capitulum with filiform florets, 4-5 mm long.

Comments. This is a very frequent species in the study area. It grows in various habitats, from deep soils of grasslands, to shallow soils and cracks of the slopes and summit. It is similar to Baccharis draunculifolia ssp. dracunculifolia, but it has linear leaves (length-width ratio $6: 3: 1-11: 6: 1$ ), and it does not occur in south-eastern Buenos Aires province.

\section{Genus Sommerfeltia Less.}

\section{Sommerfeltia spinulosa (Spreng.) Lessing}

Figure 32

Sommerfeltia spinulosa (Spreng.) Lessing (1832): 190 - Cabrera (1963): 98; Zuloaga et al. (2014a): 240; Tropicos (2017).

Conyza spinulosa Sprengel (1826): 510.
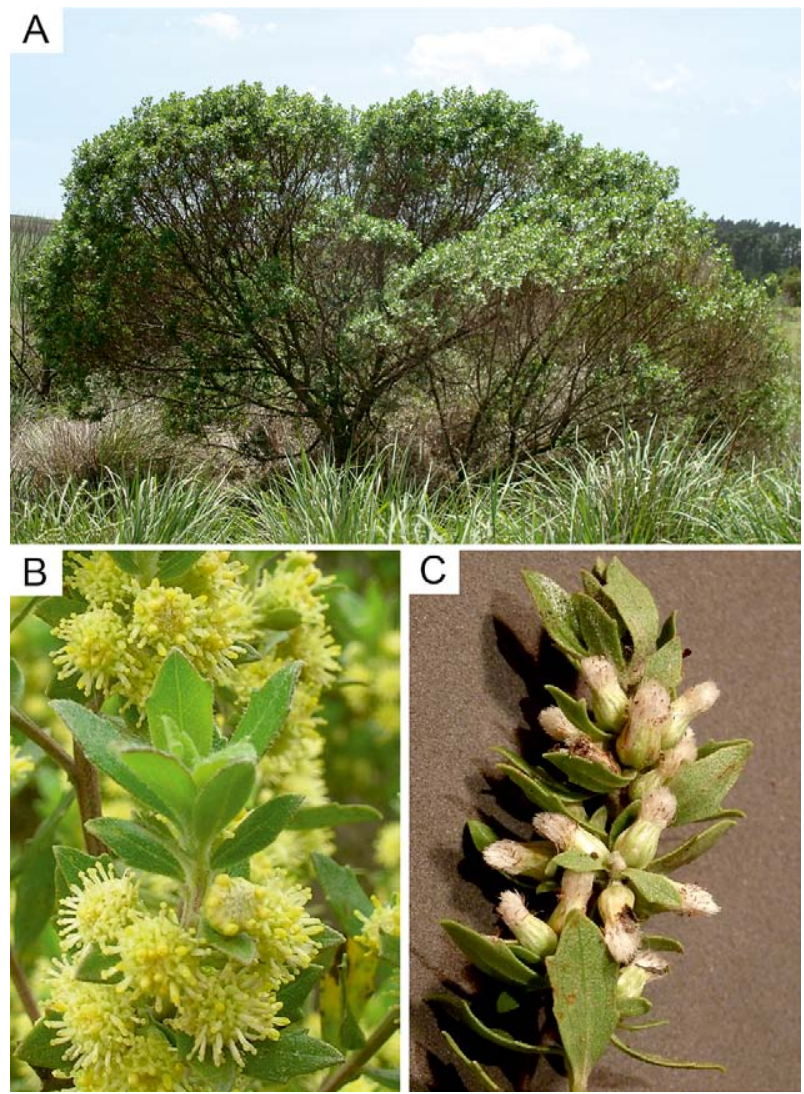

Figure 31. Baccharis dracunculifolia ssp. tandilensis. A. Plant. B. Male capitulums. C. Female capitulums.

Chamaephytes; endemic to mountain ranges of Buenos Aires province. Least Concern (Delucchi 2006).

Characteristics. Shrub with many branches, to 40 $\mathrm{cm}$ tall, with pinnatisect, spiniform and rigid leaves, 1-2.5 cm long. Capitulum radiate, bell-shaped, $7 \mathrm{~mm}$ long, with white ray outer florets and yellow tubular disc florets. Achenes with glandular and pubescent surface, 3.5-4 mm long, crowned by a white pappus.

Comments. This is an infrequent species in the study area, but it is a common species in other mountain ranges of the Tandilia system. It grows in full sunlight conditions, in the shallow soils and cracks at the summit.

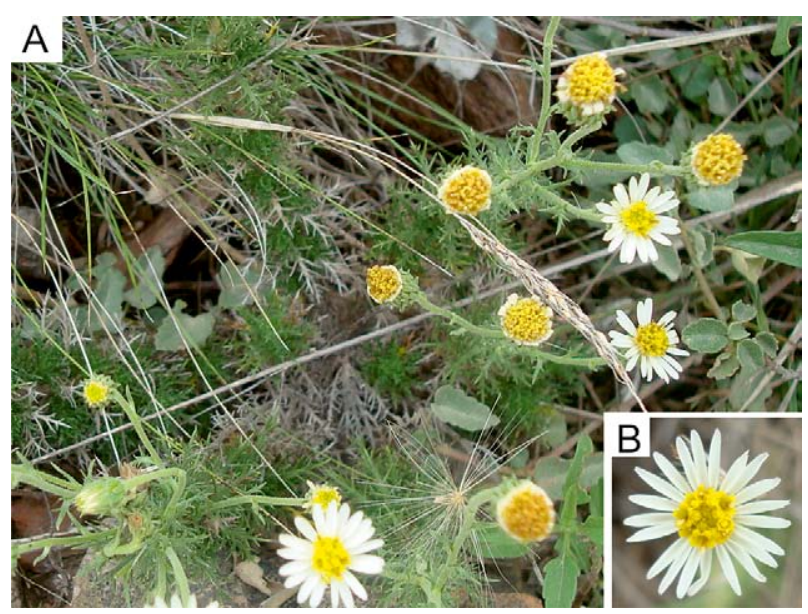

Figure 32. Sommerfeltia spinulosa. A. Plant. B. Capitulum. 
Sommerfeltia spinulosa is the only species of its genus that has been found in Argentina.

Order Asterales: Family Asteraceae: Tribe Eupatorieae

Genus Stevia L.

Stevia satureiifolia (Lam.) Sch. Bip. var. patagonica (Hieron.) Hieronymus

\section{Figure 33}

Stevia satureiifolia (Lam.) Sch. Bip. var. patagonica (Hieron.) Hieronymus (1897): 737-Cabrera (1963): 37; Cabrera and Freire (1997): 94; Zuloaga et al. (2014a): 428; Tropicos (2017).

Stevia multiaristata Spreng. var. patagonica Hieronymus (1880): 353. Stevia satureiifolia (Lam.) Schultz Bip. ex Klotzsch (1852): 291

Stevia multiaristata Sprengel (1826): 449

Eupatorium satureifolium Lamark (1786): 411.

Chamaephytes,native with distribution in the Southern Cone Region of South America. Least Concern (Delucchi 2006).

Characteristics. Suffrutescent with many branches and leaves, $20-60 \mathrm{~cm}$ tall. Leaves pubescent, linear, to $2.5 \mathrm{~cm}$ long and $2 \mathrm{~mm}$ in diameter and with obtuse apex. Capitulums: isomorph, with a brief peduncle and pinkish tubular florets, disposed in dense corymbs. Achenes: cylindrical, with glabrous surface and reddish pappus.

Comments. This is an infrequent species in the study area. It grows in full sunlight conditions, in shallow soils and deep cracks of rocks on the grassland slopes and on the summit. This species is similar to $S$. multiaristata Spreng and $S$. aristata Don but $S$. multiaristata presents long peduncles and $S$. aristata has rhombic leaves.

Order Asterales: Family Asteraceae: Tribe Gnaphaleae

Genus Gamochaeta Wedd.

\section{Gamochaeta pensylvanica (Willd.) Cabrera}

\section{Figure 34}

Gamochaeta pensylvanica (Willd.) Cabrera (1961): 376-Cabrera (1963): 175; Hurrell (2013): 157; Freire and Ihlarlegui (2014): 477; Flora Argentina (2017); Tropicos (2017).
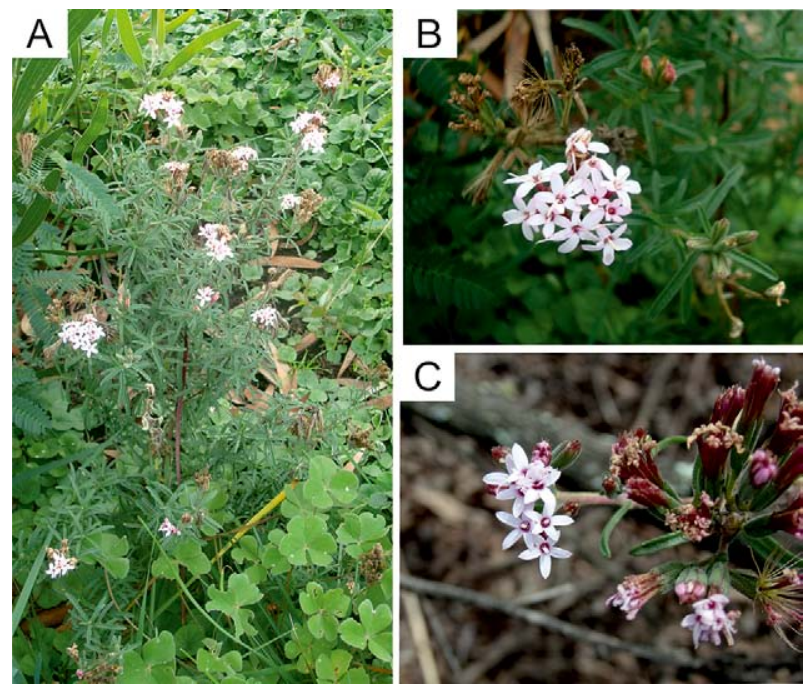

Figure 33. Stevia satureiifolia. A. Plant. B. Detail of the leaves and detail of the capitulums disposition (dense corymbs). C. Detail of the brief peduncles.

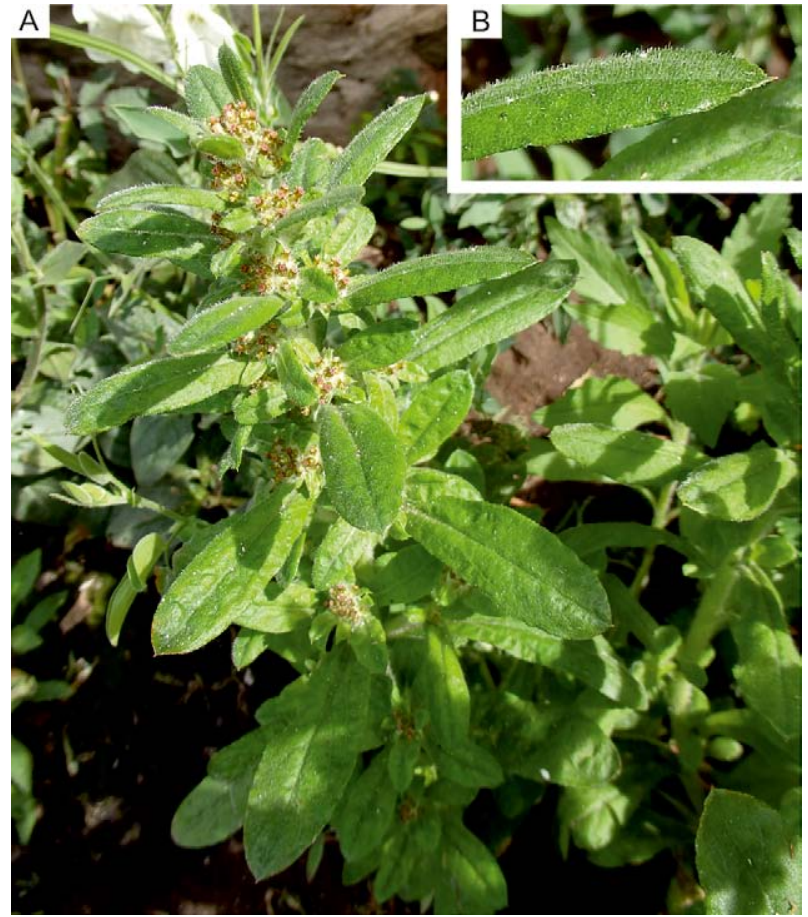

Figure 34. Gamochaeta pensylvanica. A. Plant. B. Detail of the leaf surface.

Gamochaeta platensis (Cabrera) Cabrera (1961): 376 - Cabrera (1963): 168; Hurrell (2013): 152.

Gnaphalium platense Cabrera (1941): 167.

Gnaphalium pensylvanicum Willdenow (1809): 829.

Hemicryptophyte, native with distribution in the Southern Cone Region of South America; previously recorded from Buenos Aires province but not from mountainous areas. Vulnerable, reasons unknown (Delucchi 2006).

Characteristics. Perennial herb, to $60 \mathrm{~cm}$ tall, with simple ascending branches, densely covered with leaves and lanate and glandular hairs. Leaves with spatulate shape, mucronate or obtuse apex, entire margin and attenuate to decurrent base. Leaf surface concolor or weakly bicolor with lanate and glandular hairs, denser on the abaxial surface. Inflorescences: capitulums with numerous filiform outer florets and only 3 or 4 tubular disc florets, arrange in glomerules, placed over pseudospiciform arrays interrupted by leaves. Phyllaries with lanate hairs.

Comments. This is a frequent species in the study area. It grows in intermediate and full sunlight conditions and on shallow soils and cracks on the slopes and summit. It differs from other Gamochaeta species that grow in Buenos Aires province by the leaf hair type: G. pensylvanica has lanate and glandular hairs. Cabrera (1963) and Hurrell (2013), who only noted the presence of glandular hairs in G. platensis, considered G. pensylvanica and $G$. platensis to be different species.. However, Freire and Iharlegui (2014) considered that these taxa to be synonyms.

Order Asterales: Family Asteraceae: Tribe Inuleae

Genus Pterocaulon Elliott 

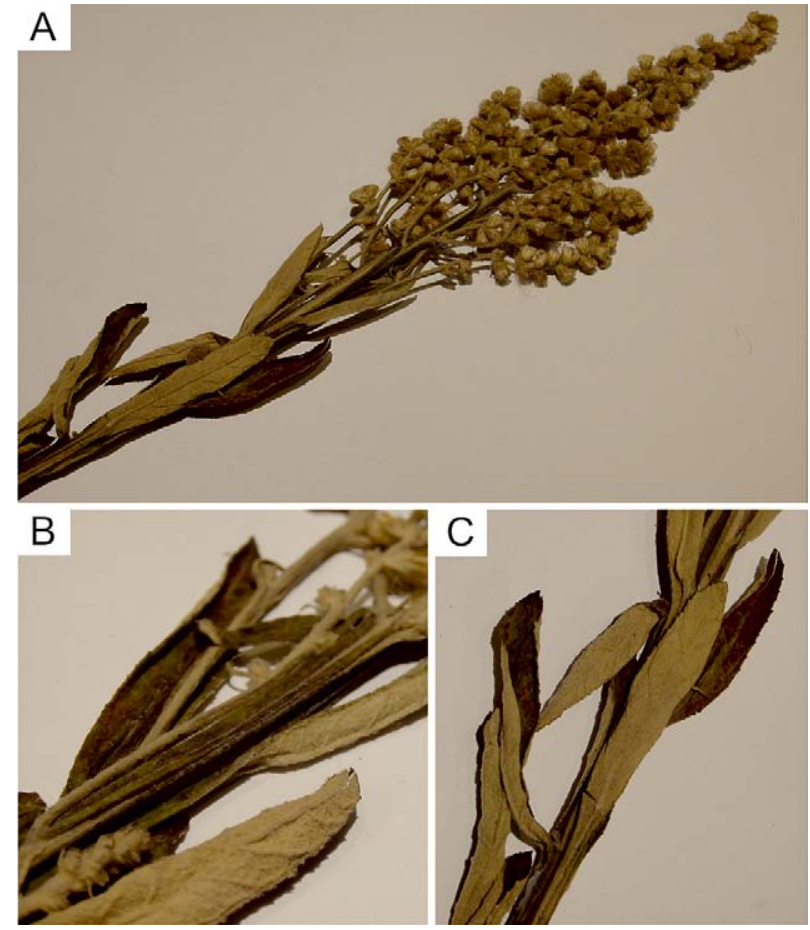

Figure 35. Pterocaulon cordobense. A. Detail of the inflorescence. B. Detail of the winged stem. C. Detail of the leaves.

\section{Pterocaulon cordobense Kuntze}

Figure 35

Pterocaulon cordobense Kuntze (1898): 169—Cabrera (1963): 141; Hurrell (2013): 190; Flora Argentina (2017); Tropicos (2017).

Hemicryptophytes, native with wide distribution in the Southern Cone Region of South America, previously recorded in Buenos Aires province but not in mountainous areas.

Characteristics. Perennial herb with brief xylopodium, to $80 \mathrm{~cm}$ tall, branches tomentouse to glabrous. Leaves with ovate to lanceolate shape, acute apex, dentate edge and decurrent base, giving the appearance of having winged stems. Abaxial surface of the leaves tomentouse; adaxial surface glabrous. Inflorescence: capitulums with numerous filiform outer florets and only 2 or 3 white tubular disc florets. Achenes with glandular-pubescent surface.

Comments. This is a rare species in the study area. It grows in full sunlight conditions, on deep soils and in cracks of the slopes. It differs from other Pterocaulon species that grow in Buenos Aires province by being less than $100 \mathrm{~cm}$ tall, and by having dentage, oblanceolate leaves as well as inflorescence with branched spikes.

Order Asterales: Family Asteraceae: Tribe Lactuceae

Genus Hypochaeris L.

\section{Hypochaeris neopinnatifida C.F. Azevedo-Gonçalves \& Matzenbacher}

Figure 36

Hypochaeris neopinnatifida C.F. Azevedo-Gonçalves \& Matzenbacher (2006): 158-Flora Argentina (2017); Tropicos (2017).

Hypochaeris rosengurttii Cabr. var. pinnatifida (Speg.) Cabrera (1941): 393-Cabrera (1963): 397.

Hypochaeris variegata (Lam.) Baker var. pinnatifida Spegazzini (1896): 40

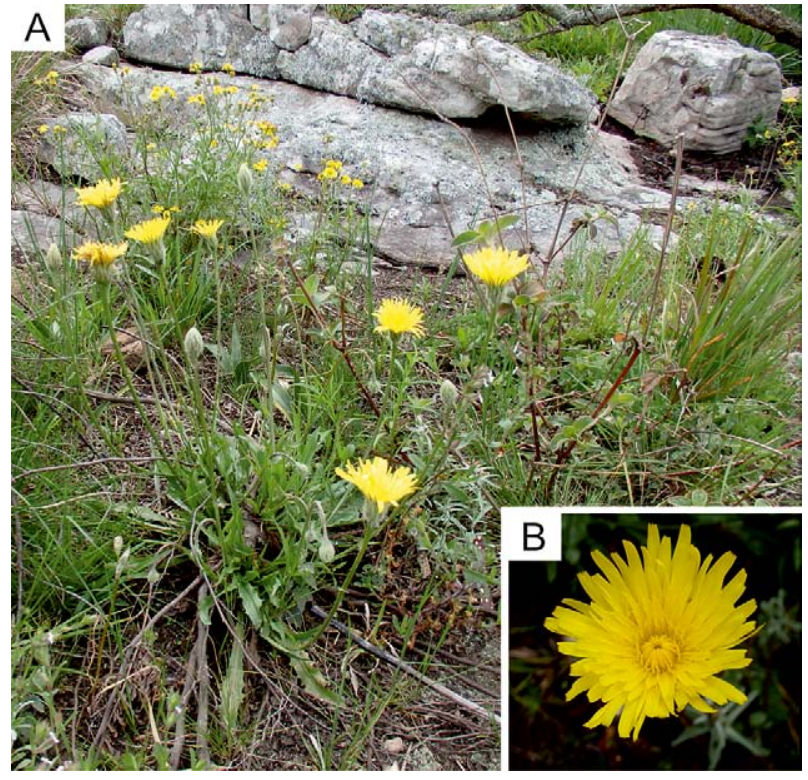

Figure 36. Hypochaeris neopinnatifida. A. Plant. B. Capitulum.

Hieracium variegatum Lamark (1786): 362.

Cryptophytes; native with wide distribution in the Southern Cone Region of South America. Vulnerable, reasons unknown (Delucchi 2006).

Characteristics. Perennial herb, with latex, rhizomes and simple or bifurcated erect stems, 10-40 cm tall. Leaves arrange in a basal rosette, with oblanceolate shape, lobate to pinnatisect margin, attenuate base and acute apex. Inflorescence: solitary discoid capitulums with yellow florets. Achenes with a short thick beak, ca $0.5 \mathrm{~mm}$ in diameter, crowned by a pappus with 1 -series plumose hairs.

Comments. This is an infrequent species in the study area. It grows in full sunlight conditions in shallow soils at the summit. Hypochaeris neopinnatifida and H. pampasica Cabrera differ from the other Hypochaeris species in Buenos Aires province by having achenes with beaks that are shorter than the seminiferous portion, and a 1-series pappus hair disposition. Particularly, H. pampasica has a thinner beak than $H$. neopinnatifida.

Genus Hieracium L.

\section{Hieracium tandilense Sleumer}

Figure 37

Hieracium tandilense Sleumer (1956): 97—Cabrera (1963): 432; Flora Argentina (2017); Tropicos (2017).

Cryptophytes; endemic to mountain ranges of Buenos Aires province. Critically Endangered, reasons unknown (Delucchi 2006).

Characteristics. Perennial herb with latex; rhizomes erect; stems ramified and lanose, to $60 \mathrm{~cm}$ tall. Leaves in a basal rosette, with lanceolate shape, ca $12 \mathrm{~cm}$ long, $3 \mathrm{~cm}$ in diameter, caulinar leaves smaller and without rigid or stiff bristles. Inflorescence: discoid capitulums with yellow florets in a cymose-corymb inflorescence. The phyllaries have lanuginose hairs mixed with black, short, soft, glandular hairs. Black-red achenes crowned by a light brown pappus. 

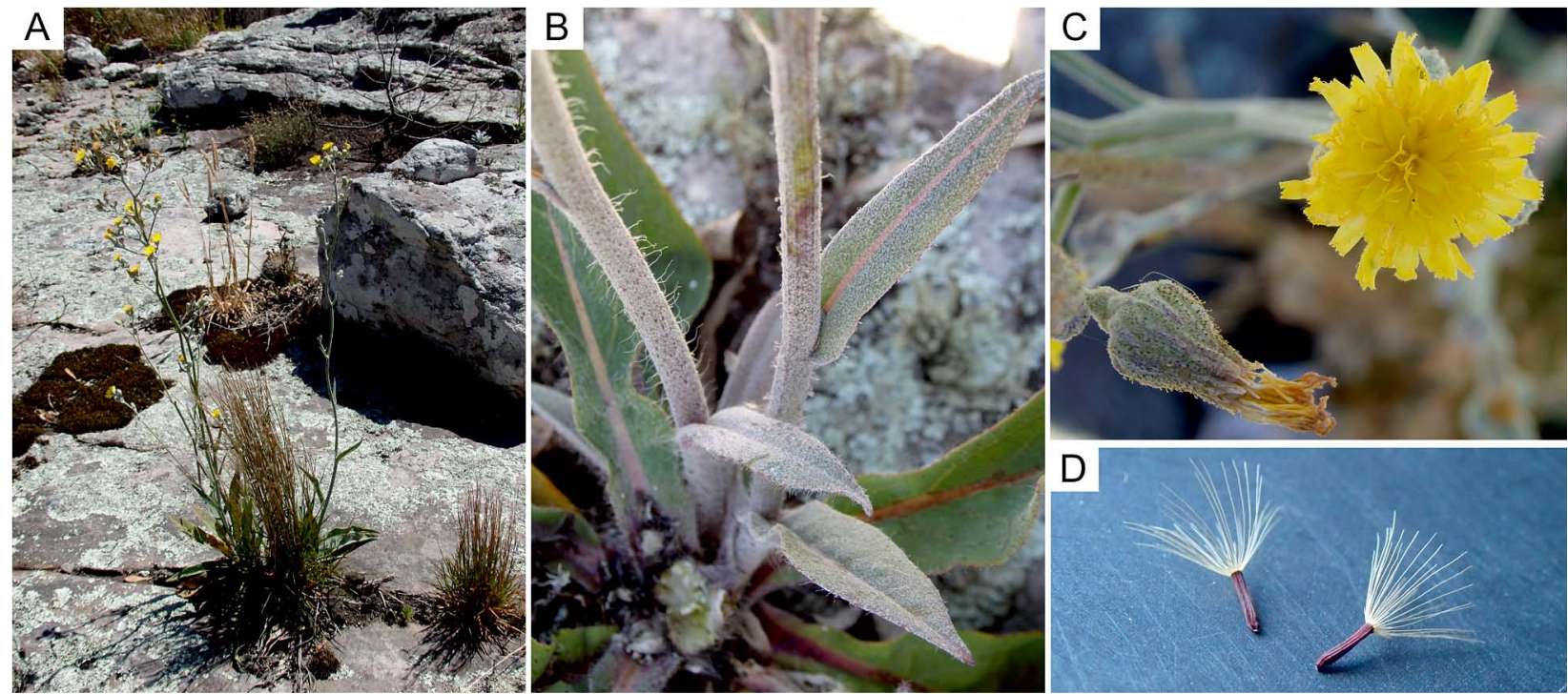

Figure 37. Hieracium tandilense. A. Plant: B. Detail of stem and leaves. C. Capitulums and phyllaries. D. Achens.

Comments. This is a rare species in the study area. It grows in full sunlight conditions in cracks at the summit. Hieracium palezieuxii Zahn and H. burkartii Sleumer also occur in the region but $H$. palezieuxii presents rigid and stiff bristles on the stems and leaves, while $H$. burkartii has rigid bristles on the phyllaries.

Order Asterales: Family Asteraceae: Tribe Mutisieae Genus Chaptalia Vent.

\section{Chaptalia piloselloides (Vahl.) Baker}

\section{Figure 38}

Chaptalia piloselloides (Vahl) Baker, ex: Martius (1884): 378-Cabrera (1963): 366; Flora Argentina (2017); Tropicos (2017). Perdicium piloselloides Vahl (1791): 38.

Cryptophytes; native with wide distribution in the Southern Cone Region of South America.

Characteristics. Perennial herb with fascicled thick roots. Leaves in a basal rosette, green in the adaxial surface and white in the abaxial one, $0.7-1.5 \mathrm{~cm}$ wide, with oblanceolate shape, retrorsely-dentate margin, acute apex and long attenuate base ending in a sheathed petiole. Inflorescence: solitary discoid capitulums at the end of a lanate peduncle, with glabrous phyllaries and three kinds of white florets; ray outer florets (female flowers), filiform intermediate florets (female flowers) and bilabiate disc florets in the center of the head (hermaphrodite flowers). Achenes with a beak and crowned by a reddish pappus.

Comments. This is an infrequent species in the study area. It grows in shallow to moderately deep, damp soils of the summit and grassland slopes. Chaptalia exscapa (Pers.) Baker is a species frequently found in the Tandilia mountain range, but it differs from $C$. piloselloides by the 2 to $4 \mathrm{~cm}$ wide leaves, achenes without beak, and lack of scape when flowering.

Order Asterales: Family Asteraceae: Tribe Senecioneae Genus Senecio L.

\section{Senecio grisebachii Baker var. subincanus Cabrera}

Figure 39

Senecio grisebachii Baker var. subincanus Cabrera (1950): 70Cabrera (1963): 315; Hurrell (2013): 242; Zuloaga et al. (2014b):

123; Tropicos (2017).

Senecio grisebachii Baker, ex Martius (1884): 313.

Chamaephytes; endemic to mountain ranges of Buenos Aires province.

Characteristics. Perennial herb with erect stems, to 1 $\mathrm{m}$ tall. Leaves with lanceolate shape, acute apex, attenuate base and serrate margin. Adaxial leaf surface lanose to glabrous; abaxial leaf surface tomentose, white. Inflorescence: radiate capitulums with ray yellow outer florets,
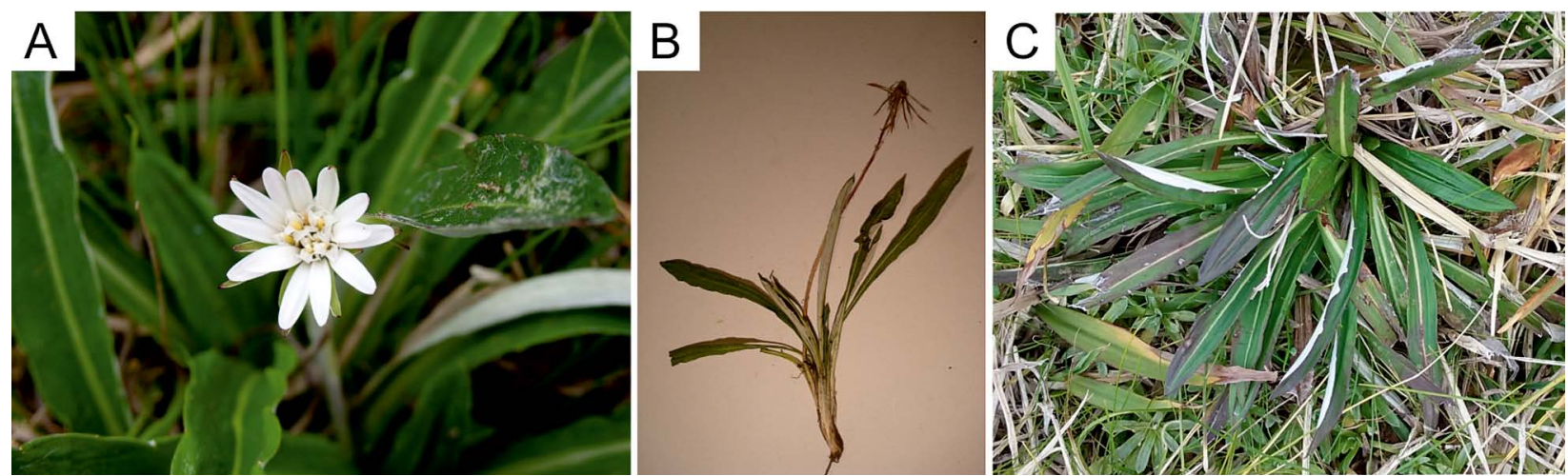

Figure 38. Chaptalia piloselloides. A. Capitulum. B, C. Plant. 


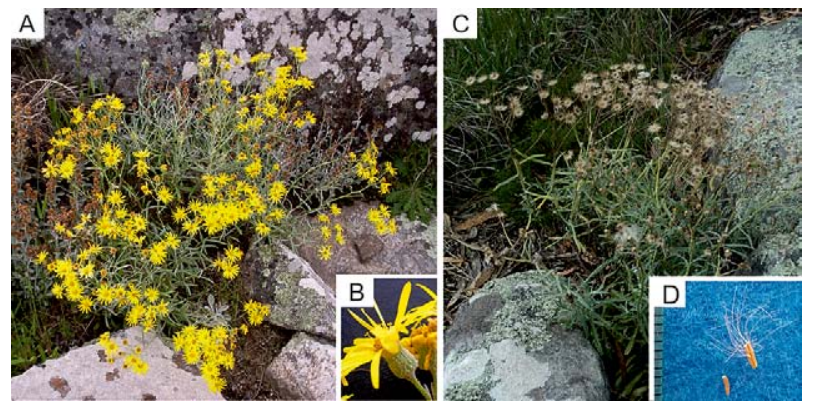

Figure 39. Senecio grisebachii var. subincanus. A. Plant at blooming. B. Capitulum. C. Plant after fructification. D. Achenes with and without pappus.

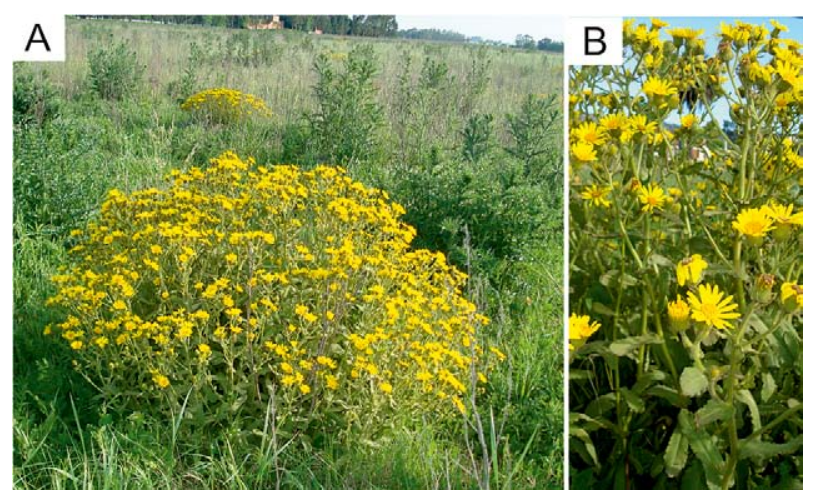

Figure 40. Senecio selloi. A. plant. B. steams at blooming.

and yellow tubular disc florets disposed in cymose-cor$y m b$ inflorescences at the end of the branches; phyllaries densely covered with white tomentose hairs. Fruits: achenes with sericeous pubescent surface and white pappus.

Comments. This is an infrequent species in the study area. It grows in full sunlight conditions in cracks on the summit. Senecio grisebachii var. subincanus is the only variety of $S$. grisebachii that has been recorded in the region. It can be recognized by its tomentose, not glandular hairs and its pubescent achenes.

\section{Senecio selloi (Spreng.) DC}

Figure 40

Senecio selloi (Spreng.) De Candolle (1837): 419-Cabrera (1963):

292; Hurrell (2013): 231; Zuloaga et al. (2014b): 192; Tropicos (2017).

Senecio doroniciflorus De Candolle (1837): 385.

Cineraria selloi Sprengel (1826): 549.

Chamaephytes; native with wide distribution in the Southern Cone Region of South America. Least Concern (Delucchi 2006).

Characteristics. Suffrutescent, to $1 \mathrm{~m}$ tall, with numerous leaves in the base of the plant. Leaves oblanceolate-spatulate, with obtuse apex and serrated margins, densely covered with white glandular hairs. Superior plant leaves sessile, with auriculate base. Inferior leaves with an auriculated pseudo-stalk and attenuate base. Inflorescence: bell-shaped radiate capitulums with yellow ray outer florets and yellow tubular disc florets disposed in loose corymbs. Fruits: cylindrical achenes with glabrous surface and white pappus.

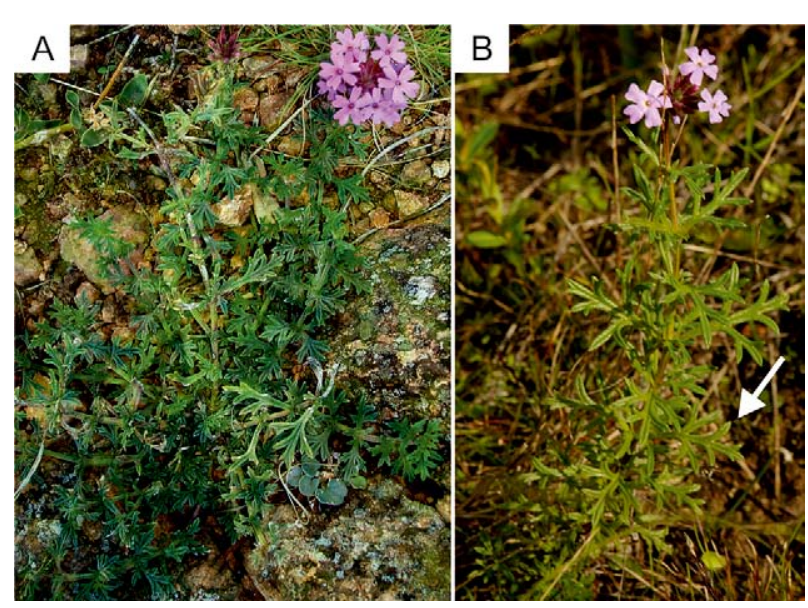

Figure 41. Glandularia tenera. A. Plant. B. Plant and leaf detail (arrow).

Comments. This is a frequent species in the study area. It grows in full sunlight conditions in deep to moderately deep soils or in deep cracks on grassland slopes. It can be recognized by its densely glandular hairs, entire or dentate leaf margin and $14-15 \mathrm{~mm}$ tall involucre.

Order Lamiales: Family Verbenaceae: Tribe Verbeneae

Genus Glandularia G. F. Gemel.

\section{Glandularia tenera (Spreng.) Cabrera}

Figure 41

Glandularia tenera (Spreng.) Cabrera (1953): 398-Flora Argentina (2017); Tropicos (2017).

Glandularia pulchella (Sweet) Tronc. var. gracilior Tronc. (1964): 470-Cabrera (1965b): 139.

Verbena tenera Spreng. (1825a): 750 - Tropicos (2017).

Hemicryptophytes; native with wide distribution in the Southern Cone Region of South America. Critically Endangered, reasons unknown (Delucchi 2006).

Characteristics. Prostrate herb with strigose pubescence in stems and floral branches. Leaves briefly petiolate with petiole less than $10 \mathrm{~mm}$. Leaf blade of 15-20 $\mathrm{mm}$ long and $15 \mathrm{~mm}$ wide, 3- to 5-dissected to bipinnatisect, segments linear to narrowly ovate and both surfaces strigose; the abaxial surface presents glands. Inflorescences: dense multifloral spikes arranged in monobotrya or pleiobotrya with frondose paracladia; peduncles $15-20 \mathrm{~mm}$ long. Floral bracts $3.5-4.5 \mathrm{~mm}$ long, ovate, with acute apex and strigose pubescence with patelliform glands; calyx $8-9 \mathrm{~mm}$ long, strigose with some patelliform glands; corolla $12-13 \mathrm{~mm}$ long, externally glabrous, lilac to violet. Fruit: cluses of 4-6 $\mathrm{mm}$ long, 1.5-2 $\mathrm{mm}$ wide, with rostrate apex.

Comments. This is a frequent species in the study area. It grows in full sunlight conditions in shallow soils on grassland slopes and at the summit. Glandularia tenera and G. peruviana (L.) Small, both commonly in southern Buenos Aires province. G. peruviana has leaves entire and the corolla red.

Order Ericales: Family Primulaceae: Tribe Lysimachieae

Genus Pelletiera A. St. Hil. 


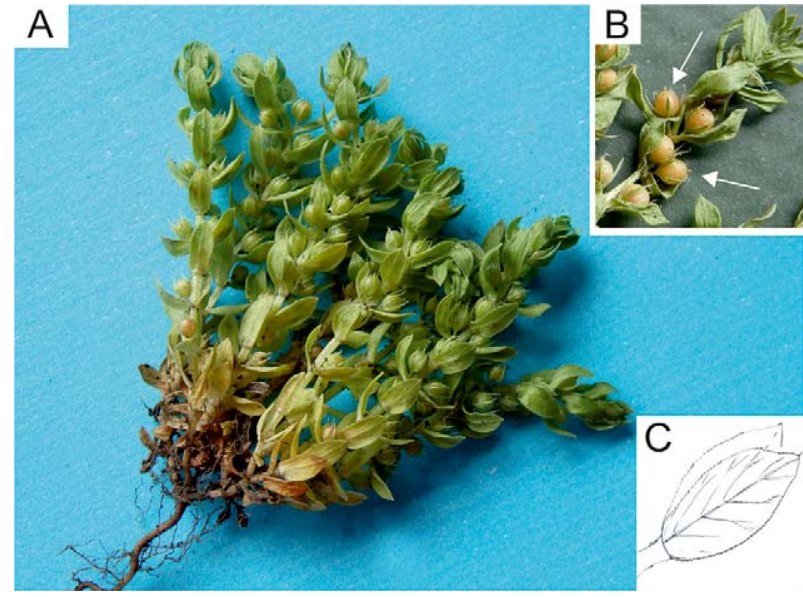

Figure 42. Pelletiera verna. A. Plant. B. Detail of fruits (arrows). C. Leaves.

\section{Pelletiera verna A. St. Hil.}

\section{Figure 42}

Pelletiera verna A. St. Hil. (1822): 365-Flora Argentina (2017); Tropicos (2017).

Pelletiera serpyllifolia (Schreb.) O. Kuntze (1898): 193-Cabrera (1965b): 7 .

Lysimachia serpyllifolia Schreber ex Poiret (1814): 477.

Therophytes, native with distribution in the Southern Cone Region of South Americ; previously recorded in Buenos Aires province but not in mountain range areas.

Characteristics. Annual, dwarf herb, to $10 \mathrm{~cm}$ tall, with angular stems. Leaves 4-6 mm long, opposite, sessile, with eliptic shape, acute apex and denticulate margin. Flowers: subsessil, 2-3 mm long; sepals linearlanceolate with apex acute; corolla white, smaller than the calyx. Fruit: globose capsule, $2 \mathrm{~mm}$ long, longitudinal dehiscent.

Comments. This is an infrequent species in the study area. It grows in full sunlight conditions in shallow soils on grassland slopes and at the summit. Pelletiera verna is the only species of its genus in Argentina.

Order Gentianales: Family Rubiaceae: Tribe Spermacoceae

Genus Borreria G. F. W. Meyer

\section{Borreria dasycephala (Chamisso \& Schlechtendal) Bacigalupo \& E.L. Cabral}

Figure 43

Borreria dasycephala (Cham. \& Schltdl.) Bacigalupo \& E.L. Cabral (1996): 306-Flora Argentina (2017); Tropicos (2017).

Diodia dasycephala Cham. \& Schltdl. (1828): 348—Cabrera (1965b) 351.

Hemicryptophytes, native with wide distribution in the Southern Cone Region of South America; previously recorded in Buenos Aires province but not in mountain range areas.

Characteristics. Perennial, rhizomatous herb with tetragonal stems. Leaves glabrous, with ovate-oblong shape, acute apex and attenuate base. Flowers: terminal or subtermianl, in groups of 1-3 flowers per peduncle; corolla and anthers white. Fruit: 2 mericarps with pubescence at the top.

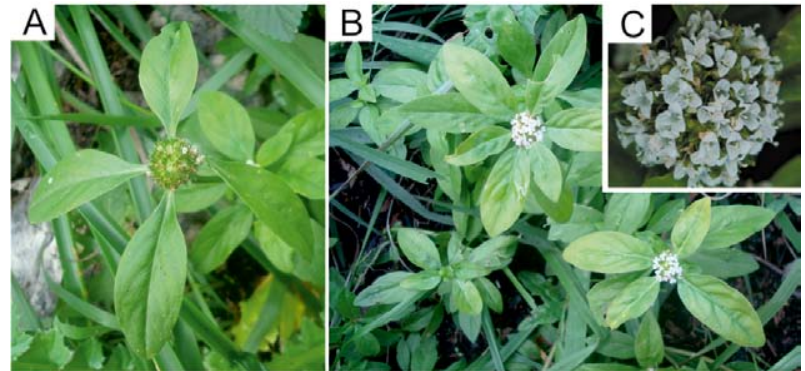

Figure 43. Borreria dasycephala. A. Leaf detail. B. Plant with terminal inflorescences. C. Flowers.

Comments. This is a very frequent species in the study area. It grows in full sunlight conditions with other short species in deep and moderately deep soils on grassland slopes and at the summit. This species has been described as an annual species (Flora Argentina 2017) contrary to the specimens seen in the Paititi Reserve, which are hemicryptophyte herbs as previously described by Cabrera (1965b) and Delprete et al. (2005).

Order Gentianales: Family Rubiaceae: Tribe Rubieae

Genus Galium L.

\section{Galium hirtum Lamarck}

Figure 44

Galium hirtum Lamark (1786): 583-Cabrera (1965b): 367; Flora Argentina (2017); Tropicos (2017).

Relbunium hirtum (Lam.) K. Schumann, ex Martius C. F. P. (1888): 107-Cabrera (1965b): 367.

Therophytes, native with distribution in the Southern Cone Region of South America.

Characteristics. Annual herb with tetragonal, pubescent stems. Leaves pubescent, ovate, with apex acute. Pseudoverticils formed by 2 leaves and 2 foliar stipules.

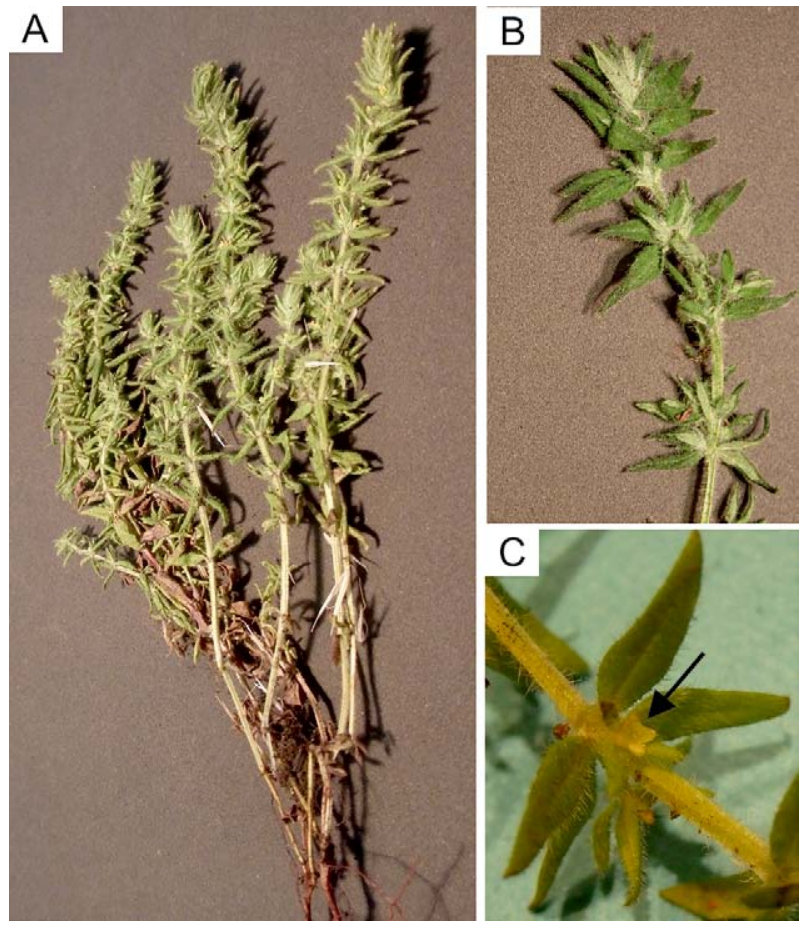

Figure 44. Galium hirtum. A. Plant. B. Detail of leaves and stems. C. Flower (arrow). 


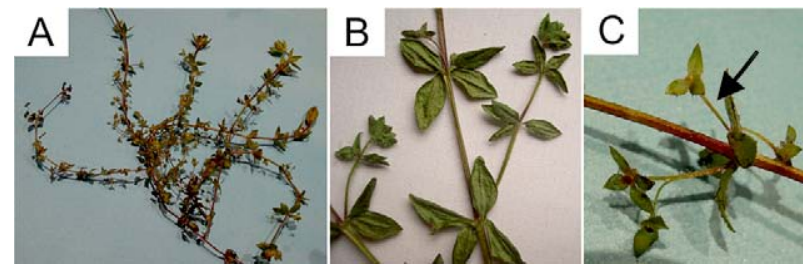

Figure 45. Galium hypocarpium. A. plant. B. Detail of leaves and stems. C. Peduncles (arrow).

Flowers: solitarly upon peduncles that are smaller than the involucral bracts; 4 yellow, small petals. Fruit: a white berry.

Comments. This is a frequent species in the study area. It grows in shaded, moist soils and under the trees. It grows with other Galium species such as G. aparine, an annual herb with sticky hairs on leaves and fruits, and $G$. hipocarpium, a perennial herb with long peduncle flowers and red berries.

\section{Galium hypocarpium (L.) Endlicher}

\section{Fig. 45}

Galium hypocarpium (L.) Endlicher, ex Grisebach (1861): 351-Flora Argentina (2017); Tropicos (2017).

Relbunium hypocarpium (L.) Hemsley (1881): 63-Cabrera (1965b): 370.

Valantia hypocarpia L. (1759): 1307

Hemicryptophytes, native with wide distribution in the Southern Cone Region of South Americ; previously recorded in Buenos Aires province but not from mountain range areas. Vulnerable (Delucchi 2006).

Characteristics. Perennial herb with tetragonal stems and thin, reflexed pubescence. Roots: fibrous, arising from inferior stem nodes. Leaves: oblong, ovateobovate, with apex acute, obtuse, or cuspidate, margin revolute , and pubescence fine. Pseudoverticils formed by 2 leaves and 2 foliar stipules. Flowers: solitarly, placed upon peduncles that are larger than the involucral bracts. Fruit: a red berry.

Comments. This is an infrequent species in the study area. It grows in damp soils, under trees and in grasslands near the summit. It is similar to G. noxium (A. St. Hil.) Dempster ssp. valantioides (Cham. \& Schltdl.) Dempster, which, however, has white berries.

Order Lamiales: Family Plantaginaceae: Tribe Plantagineae

\section{Genus Plantago L.}

\section{Plantago tandilensis (Pilg.) Rahn}

Figure 46

Plantago tandilensis (Pilg.) Rahn (1983): 337-Flora Argentina (2017); Tropicos (2017).

Plantago brasiliensis Sims var. tandilensis Pilger (1928): 64 - Cabrera (1965b): 338

Plantago brasiliensis Sims (1825): 2616.

Chamaephytes; endemic. Vulnerable, reasons unknown (Delucchi 2006).

Characteristics. Shrub with numerous ascendant branches, $20-50 \mathrm{~cm}$ tall. Leaves: glabrous or slightly pubescent, with linear shape, 3-5 veins and sheathed

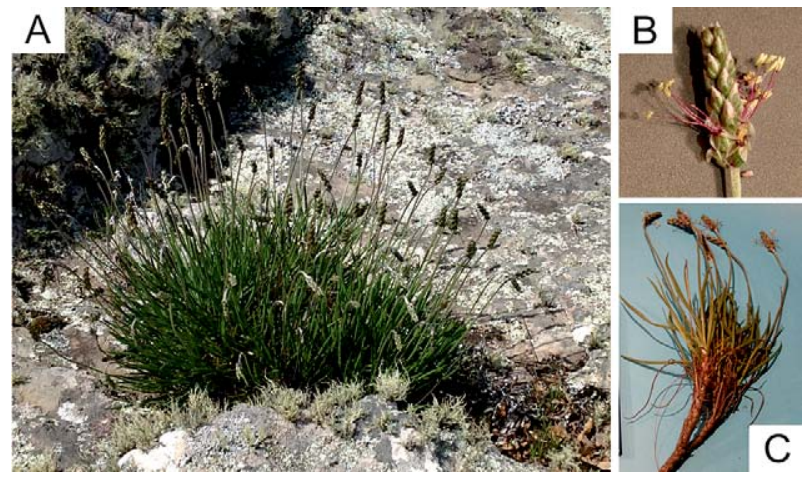

Figure 46. Plantago tandilensis. A. Plant. B. Inflorescence. C. Detail of branches.

base; 8-20 cm long and 1-3 $\mathrm{mm}$ wide. Inflorescences: cylindrical dense spikes, $1-5 \mathrm{~cm}$ long; peduncles longer than the leaves; flowers with free sepals. Fruit: a capsule with 2 seeds.

Comments: This is a rare species in the study area. It grows in full sunlight conditions in cracks on the summit. Plantago bismarckii Nederlein, a species endemic to the Ventania mountain range, has sericeous-tomentose leaves and a smaller spike than $P$. tandilensis.

\section{Discussion}

Ferns are moderately represented in the Sierra Chica; the study area had $50 \%$ of the species that Alonso et al. (2009b) reported from the Tandilia system and only $14 \%$ of the taxa reported by Capurro (1961) from the whole of Buenos Aires province. Gymnosperms were represented only by 2 adventive Pinus spp., originating from past plantations. The major taxonomic group was the angiosperms, which are mainly represented by eudicots. This is similar to what has been observed in the Ventania system (Long and Grassini 1997) and in the mountains of other Argentine provinces (Méndez 2009, Oggero and Arana 2012).

Of the 364 species recorded in this study, 71 were not included by Alonso et al. (2009b) in a review of the flora of the Tandilia system. Taking into account these 71 taxa, the floristic richness described by Alonso et al. (2009b) would rise from 578 to 649 taxa. This value is similar to the number of specific and infraspecific taxa described for other mountain ranges of Argentina, such as the Ventania system $(N=652)$, the eastern area of the Cordón del Plata in Mendoza province $(N=667)$, and the southern part of the Sierras Pampeanas Orientales in Córdoba province $(N$ = 734) (Long and Grassini 1997, Méndez 2009, Oggero and Arana 2012).

The representativeness of the surveyed botanic families $(N=72)$ was dissimilar because more than $50 \%$ of the total identified taxa was grouped into 6 families. The Asteraceae and the Poaceae accounted for $41 \%$ of the inventoried vascular plants. The predominance of these families has also been observed elsewhere in Argentina (Flora Argentina 2017) and specifically in several orographic systems in La Pampa, Córdoba, and Mendoza 
provinces (Mazzola et al. 2008, Méndez 2009, Morici et al. 2010, Oggero and Arana 2012, Cantero et al. 2014). In Buenos Aires province, the high species richness of these 2 families was previously recorded in the Ventania system (Frangi and Bottino 1995, Long and Grassini 1997), and for other mountain ranges of the Tandilia system (Escaray 2007, Alonso et al. 2009a, 2009b, Álvarez et al. 2012).

All species identified in this study had already been reported for Buenos Aires province (Cabrera 1963, 1965a, 1965b, 1967, 1968, 1970, Flora Argentina 2017), with the exception of Nothoscordum gaudichaudianum Kunth. However, not all species were reported from mountain habitats (Frangi 1975, Long and Grassini 1997, Valicenti et al. 2000, 2005, Escaray 2007, Alonso et al. 2009a, Álvarez et al. 2012), and most of these are species that frequently thrive in disturbed and agricultural landscapes of southeastern Buenos Aires province (Montes et al. 2006). Others are well-known weeds, ornamental trees, fruit shrubs, forage plants, or water plants (Dimitri 1978, Alonso and Ispizúa 1996, 2008, Lahitte et al. 2004, Montes et al. 2006, Hurrell 2013, Ahumada et al. 2016).

Therophytes from the Paititi Reserve represented about a third of all taxa found and were mostly adventive species. Among the perennial species, cryptophytes and hemicryptophytes were the most abundant life forms. In these 2 categories, plants keep their buds beneath or at the soil surface, which provides considerable protection against adverse conditions such as shallow soils, high exposure to solar radiation, seasonal drought, and high summer temperatures, conditions that also increase fire danger (Frangi 1975, Mazzola et al. 2008).

According to Zalba and Villamil (2002), the process of invasion by adventive species is dynamic and some species classified as casual and non-invasive can become aggressive invaders due to increases in the number of propagules, changes in the regime of disturbance, and the introduction of other species that may facilitate their expansion. Accordingly, the presence of several exotic tree and shrub species (e.g., Acacia melanoxylon, Laurus nobilis, Prunus mahaleb, and Rubus ulmifolius) as well as some herbaceous species (e.g., Holcus lanatus, Senecio madagascariensis, Cirsium vulgare, and Carduus spp.) that grow spontaneously in different parts of the reserve, may become invasive and thus contribute to the deterioration of natural areas and the loss of native populations (Isla et al. 2001, Monserrat 2010).

The reserve hosted $60 \%$ of the native species reported for the Tandilia system by Alonso et al. (2009b), and at least $4 \%$ of the endemisms reported for Argentina by Zuloaga and Morrone (1996, 1999), some of which are unique to the Tandilia system, like Baccharis dracunculifolia ssp. tandilensis, Hieracium tandilense, and Plantago tandilensis; others, like Cypella herbertii subsp. wolffhuegeli, Mimosa rocae, Poa iridifolia, and Vicia setifolia var. bonariensis, are also found in the Ventania system (Crisci et al. 2001). These results reveal the remarkable richness of the native flora of the untouched area of the Paititi Reserve. This flora provides shelter and food for numerous vertebrates and invertebrates (Vega and Bellagamba 1990, Cicchino and Farina 2007), and the reserve serves to protect genetic resources and provides many ecosystem services (Echeverria et al. 2015).

Finally, this study demonstrates the importance of preserving the mountain range areas of Buenos Aires province, and particularly of this reserve, especially considering that $8 \%$ of the inventoried species are threatened species in Buenos Aires province (Delucchi 2006). The adventive species in the Paititi Reserve must be identified and quantified (i.e., density and distribution) to develop mitigation strategies. Native plant species could be used to recolonize and recover degraded sites on mountain ranges in the area. This ecosystem must be preserved to protect its natural resources, allow the conservation of native species, and maintain its role in providing ecosystem services.

\section{Acknowledgements}

We are very grateful to the Neotropical Grassland Conservancy (NGC) and to the National University of Mar del Plata (Facultad de Ciencias Exactas y Naturales, EXA 745/15; Facultad de Ciencias Agrarias, AGR 448/14$503 / 16$ ) for their financial support. We would also like to thank the engineer and anthropologist Esteban González Zugasti, owner of the Paititi Natural Reserve, for his invaluable collaboration; Ph.D. Nicolás Wyngaard and translator Verónica Leofanti for reviewing and editing the manuscript; and Lic. Hernán Angelini for helping us find the satellite image.

\section{Authors' Contributions}

MLE, SIA and VMC contributed to the study design, collected the data and wrote the text; MLE and SIA identified specimens and made the analysis.

\section{References}

Ahumada $\mathrm{OH}$, Alonso S, Amuchástegui A, Andrada A, Braun K, Cáceres E, Cantero J, Carbone L, Chaila S, Cipriotti PA, Collantes MB, D'Alfonso C, Mario N, Villalobos ME, Delucchi G, Echeverría ML, Escartin C, Galetti L, Gil ME, Giunti S, González A, Hurrell JA, Leaden MI, Lobato Echeverría RA, Long MA, López MG, Mattenella M, Maturo HM, Mulko J, Núñez C, Oakley L, Palou D, Prado DE, Puntieri J, Rauber RB, Roncaglia R, Ruiz G, Scaramuzzino R, Sobrero MT, Troiani H, Vanni O, Villamil CB (2016) Descripción de las especies, In: Fernández O, Leguizamón ES, Acciaresi HA (Eds) Malezas e Invasoras de la Argentina. Tomo II: Identificación y Reconocimiento. Editorial Universidad Nacional del Sur, Bahía Blanca, 53-935.

Alonso SI, Guma IR, Nuciari MC, Van Olphen A (2009a) Flora de un área de la Sierra La Barrosa (Balcarce) y fenología de especies nativas con potencial ornamental. Revista de la Facultad de Ciencias Agrarias, Universidad Nacional de Cuyo 41 (2): 23-44.

Alonso SI, Ispizúa VN (1996) Malezas Graminiformes de Cultivos y Parques. Clave para determinar las especies más frecuentes en el Sudeste Bonaerense. Estación Experimental Agropecuaria Balcarce, Instituto Nacional de Tecnología Agropecuaria, Balcarce, 43 pp. 
Alonso SI, Ispizúa VN (2008) Catálogo del Material del Herbario BAL: Poáceas (Gramíneas), $2^{\circ}$ edición. Instituto Nacional de Tecnología Agropecuaria, Balcarce: 116 pp.

Alonso SI, Ispizúa V, Nuciari MC, Clausen A, Calandroni M (2009b) Valor actual y potencial de los recursos florísticos del sistema serrano de Tandilia (Buenos Aires, Argentina). In: Seguel I, León P, Muñóz G, Piñeira J, Avendaño L (Eds) Proceedings $7^{\circ}$ Simposio de Recursos Genéticos para América Latina y el Caribe. Instituto Nacional de Investigaciones Agropecuarias, Pucón, 453-454.

Al-Shehbaz IA, Salariato DL (2012) Flora Argentina. Flora Vascular de la República Argentina. Dicotyledoneae, Brassicaceae. 8. Instituto de Botánica Darwinion-Instituto Multidisciplinario de Biología Vegetal, Consejo Nacional de Investigaciones Científicas y Técnicas, Córdoba, 270 pp.

Álvarez MF, Fernández Honaine M, Borrelli N, Osterrieth M (2012) Diversidad vegetal en canteras de áridos del sudeste bonaerense. In: del Río JL, De Marco SG (Eds) Sustentabilidad de la Minería en Áreas Periurbanas: una aproximación multidimensional. Universidad Tecnológica Nacional de Mar del Plata, Mar del Plata, 83-99.

Arduino P (1764) Animadversionum Botanicarum Specimen Alterum. Typographia Sansoniana, Venecia, $42 \mathrm{pp}$.

Arechavaleta J (1900) Flora uruguaya: Oxalideas. Anales del Museo Nacional de Montevideo 3: 201-240.

Ariza Espinar L (2005) Pródromo de la flora fanerogámica de la Argentina Central. Familia Asteraceae I. Tribu Astereae 3. Museo Botánico, Universidad Nacional de Córdoba, Córdoba, 139 pp.

Azevêdo-Gonçalves CF, Matzenbacher NI (2006) Notas nomenclaturais em Hypochaeris L. (Asteraceae). Pezquisas, Serie Botanica 57: $157-160$

Bacigalupo NM, Cabral EL (1996) Infrageneric classification of Borreria (Rubiaceae-Spermacoceae) on the basis of American species. Opera Botanica Belgica 7: 297-308.

Barboza GE (2013) Flora Argentina. Flora vascular de la República Argentina. Dicotyledoneae, Solanaceae 13: 1-349. Instituto de Botánica Darwinion-Instituto Multidisciplinario de Biología Vegetal, Consejo Nacional de Investigaciones Científicas y Técnicas, Córdoba.

Beauverd G (1906) Une liliacée nouvelle de l'Uruguay. Bulletin de 1'Herbier Boissier (sér. 2) 6: 1011

Beauverd G (1908) Nouvelles espèces uruguayennes du genre Nothoscordum Kunth. Bulletin de l'Herbier Boissier (sér. 2) 8: 93-1007.

Bennett AW (1879) Polygalæ americanæ novæ vel parum cognitæ. Journal of Botany, British and Foreign 17: 201-207.

Benton TG, Vickery JA, Wilson JD (2003) Farm and biodiversity: is habitat heterogeneity the key? Trends in Ecology and Evolution 18 (4): 182-188. https://doi.org/10.1016/S0169-5347(03)00011-9

Bertonatti C, Corcuera J (2000) Situación Ambiental Argentina 2000. Fundación Vida Silvestre, Buenos Aires, 437 pp.

Bilenca D, Miñarro F (2004) Identificación de Áreas Valiosas de Pastizal (AVP's) en las Pampas y Campos de Argentina, Uruguay y Sur de Brasil. Fundación Vida Silvestre Argentina, Buenos Aires, $323 \mathrm{pp}$.

Britton NL, Rose JN (1922) The Cactaceae, descriptions and illustrations of plants of the cactus family 3 Gibson Brothers, Washington DC, 255 pp. https://doi.org/10.5962/bhl.title.46288

Burkart AE (1966) Notas sobre las especies de Vicia (Leguminosae) del área mesopotámico-pampeana. Darwiniana 14 (1): 161-194.

Burret M (1941) Myrtaceen-Studien. Notizblatt des Botanischen Gartens und Museums zu Berlin 15 (3): 479-550.

Cabrera AL (1941) Compuestas bonaerenses. Revista del Museo de La Plata, Sección Botánica 4: 1-450.

Cabrera AL (1950) Notes on the Brazilian Senecioneae. Brittonia 7 (2): 53-74.

Cabrera AL (1953) Manual de la flora de los alrededores de Buenos Aires. Editorial Acme, Buenos Aires, 589 pp.

Cabrera AL (1961) Observaciones sobre las Inuleae-Gnaphalineae (Compositae) de América del Sur. Boletín de la Sociedad Argentina de Botánica 9: 359-386.
Cabrera AL (1963) Compuestas. Flora de la Provincia de Buenos Aires. Colección Científica Instituto Nacional de Tecnología Agropecuaria. Tomo 4 (6). Instituto Nacional de Tecnología Agropecuaria, Buenos Aires, 443 pp.

Cabrera AL (1965a) Oxalidáceas a Umbelíferas. Flora de la Provincia de Buenos Aires. Colección Científica Instituto Nacional de Tecnología Agropecuaria. Tomo 4 (4). Instituto Nacional de Tecnología Agropecuaria, Buenos Aires, 417 pp.

Cabrera AL (1965b) Ericáceas a Caliceráceas. Flora de la Provincia de Buenos Aires. Colección Científica Instituto Nacional de Tecnología Agropecuaria. Tomo 4 (5). Instituto Nacional de Tecnología Agropecuaria, Buenos Aires, 434 pp.

Cabrera AL (1967) Piperáceas a Leguminosas. Flora de la Provincia de Buenos Aires. Colección Científica Instituto Nacional de Tecnología Agropecuaria. Tomo 4 (3). Instituto Nacional de Tecnología Agropecuaria, Buenos Aires, 673 pp.

Cabrera AL (1968) Gimnospermas y Monocotiledóneas (excepto Gramíneas). Flora de la Provincia de Buenos Aires. Pteridáceas. Colección Científica Instituto Nacional de Tecnología Agropecuaria. Tomo 4 (1). Instituto Nacional de Tecnología Agropecuaria, Buenos Aires, 625 pp.

Cabrera AL (1970) Gramíneas. Flora de la Provincia de Buenos Aires. Colección Científica Instituto Nacional de Tecnología Agropecuaria. Tomo 4 (2). Instituto Nacional de Tecnología Agropecuaria, Buenos Aires, $627 \mathrm{pp}$.

Cabrera AL, Freire SE (1997) Eupatorieae (exc. Mikania). Flora Fanerogámica Argentina 47: 3-54, 76-104.

Cabrera AL, Zardini EM (1978) Manual de la Flora de los alrededores de la Provincia de Buenos Aires. Editorial Acme, Buenos Aires, $755 \mathrm{pp}$.

Calviño CI, Martínez SG (2007) Nuevas citas para Argentina y Uruguay, y notas sobre Eryngium sect. Panniculata (Apiaceae). Darwiniana 45 (1): 68-76.

Cantero J, Sfragulla J, Núñez C, Mulko J, Bonalumi A, Amuchastegui A, Barboza G, Chiarini F, Ariza Espinar L (2014) Vegetación de afloramientos carbonáticos de montañas del centro de Argentina. Boletín de la Sociedad Argentina de Botánica 49 (4): 559-580.

Capurro R (1961) Las pteridofitas de la Provincia de Buenos Aires e Isla Martín García. Anales de la Comisión de Investigaciones Científicas 2: 55-322.

Cavanilles AJ (1799) Icones et Descriptiones Plantarum quae aut sponte in Hispania crescunt, aut in hortis hospitantur 5. Ex Regia Typographia, Madrid, 776 pp.

Chamisso LKA, Schlechtendal DFL (1828) De plantis in expeditione speculatoria Romanzoffi ana observatis. Linnaea 3 (4): 338-366.

Chase MW, Reveal JL (2009) A phylogenetic classification of the land plants to accompany APG III. Botanical Journal of the Linnean Society 161 (2): 122-127. https://doi.org/10.1111/j.10958339.2009.01002.x

Chebez JC (2005) Guía de las Reservas Naturales de la Argentina 3. Editorial Albatros, Buenos Aires, 192 pp.

Christenhusz M, Reveal J, Farjon A, Gardner MF, Mill RR, Chase MW (2011b) A new classification and linear sequence of extant gymnosperms. Phytotaxa 19: 55-70. https://doi.org/10.11646/ phytotaxa.19.1.3

Christenhusz M, Zhang X.-C., Schneider H (2011a) A linear sequence of extant families and genera of lycophytes and ferns. Phytotaxa 19: 7-54. https://doi.org/10.11646/phytotaxa.19.1.2

Cichino AC, Farina JL (2007) Los carábidos (Insecta, Coleoptera) de los suelos serranos y periserranos de las estancias Paititi y El Abrojo, Sierra De Difuntos, Partido de General Pueyrredón, Provincia de Buenos Aires, Argentina. In: Proceedings VI Encuentro Nacional Científico Técnico de Biología del Suelo y IV Encuentro sobre Fijación Biológica del Nitrógeno. Universidad Nacional de Río Cuarto, Río Cuarto, 1-15.

Cisternas MA, Salazar GA, Verdugo G (2012) Transfer of Geoblasta pennicillata to Bipinnula (Chloraeinae, Orchidaceae). Phytotaxa 64: 9-10. https://doi.org/10.11646/phytotaxa.64.1.2 
Correa M (1968) Rehabilitación del género Geoblasta Barb. Rodr Revista del Museo de La Plata, Sección Botánica 11: 69-74.

Crisci VJ, Freire ES, Sancho G, Katinas L (2001) Historical biogeography of the Asteraceae from Tandilia and Ventania mountain ranges (Buenos Aires, Argentina). Caldasia 23 (1): 21-41.

De Candolle AP (1836) Prodromus Systematis Naturalis Regni Vegetabilis 5. Treuttel et Würtz, Paris, 706 pp.

De Candolle AP (1837) Prodromus Systematis Naturalis Regni Vegetabilis 6. Treuttel et Würtz, Paris, $687 \mathrm{pp}$.

Delprete PG, Smith LB, Klein RM (2005) Rubiáceas. In: Reis A (Ed.) Flora Ilustrada Catarinense. Herbário Barbosa Rodrigues, Itajaí, 349-842.

Delucchi G (2006) Las especies vegetales amenazadas de la Provincia de Buenos Aires: una actualización. Aprona Boletín Científico 39: 19-31.

Dimitri MJ (1978) Enciclopedia argentina de agricultura y ganadería. Descripción de las plantas cultivadas 1 (2). Editorial Acme, Buenos Aires, 1028 pp.

Don G (1832) A General History of the Dichlamydeous Plants 2. Gilbert \& Rivington, London, 875 pp. https://doi.org/10.5962/bhl.title.502

Echeverría ML, Comparatore VM, Alonso SI (2015) Contribución de las Especies Vasculares de la Reserva Natural Paititi (Bs. As., Argentina) al bienestar humano. $4^{\circ}$ Congreso Internacional de Servicios Ecosistémicos en los Neotrópicos: de la Investigación a la Acción. http://www.geap.com.ar/cisen4/libro-resumenes. Accessed on: 20179-23.

Elliott S (1823) Sketch of the Botany of South-Carolina and Georgia 2. J.R. Schenck, Charleston, 743 pp. https://doi.org/10.5962/bhl. title. 9508

Escalante M (1946) Las ramnáceas argentinas. Boletín de la Sociedad Argentina de Botánica 1 (3): 209-231.

Escaray FJ (2007) Estudio Florístico de una Ladera de la Sierra del Volcán (Sistema de Tandilia). Agricultural Engineering Thesis, Facultad de Ciencias Agrarias, Universidad Nacional de Mar de Plata, Buenos Aires, 98 pp.

Falasca S, Ulberich A, Bernabé N, Mordenti S (2000) Principales características agroclimáticas del sudeste bonaerense, República Argentina. Revista Geográfica 127: 91-102.

Flora Argentina (2017) Plantas vasculares de la República Argentina. http://www.floraargentina.edu.ar. Accessed on: 2017-9-25.

Frangi J (1975) Sinopsis de las comunidades vegetales y el medio de las sierras de Tandil (Provincia de Buenos Aires). Boletín de la Sociedad Argentina de Botánica 16 (4): 293-318.

Frangi J, Granco M, Sánchez N, Vicari LR, Rovetta GS (1980) Efecto del fuego sobre la composición y dinámica de la biomasa de un pastizal de Sierra de la Ventana (Bs. As., Argentina). Darwiniana 22 (4): 565-585.

Frangi J, Bottino OJ (1995) Comunidades vegetales de la Sierra de la Ventana, Provincia de Buenos Aires. Revista de la Facultad de Agronomía 71 (1): 93-133.

Freire S, Iharlegui L (2014) Género Gamochaeta Wedd. In: Zuloaga FO, Belgrano M, Anton MM (Eds) Flora Argentina. Flora Vascular de la República Argentina 7 (1). Instituto de Botánica Darwinion, Consejo Nacional de Investigaciones Científicas y Técnicas, Córdoba, 463-482.

Gillies J, Hooker WJ (1829) On the species of the genus Colletia, of the natural order Rhamnae, discovered by Dr. Gillies in South America. In: Hooker WJ (Ed.) Botanical Miscellany 1. John Murray, London, 150-156.

Graham R (1830) Description of several new or rare plants which have lately flowered in the neighbourhood of Edinburgh, and chiefly in the Royal Botanic Garden.Edinburgh New Philosophical Journal 9: 183-186.

Grisebach AHR (1861) Flora of the British West Indian Islands. Lovell Reeve \& Co., 789 pp https://doi.org/10.5962/bhl.title.143

Grondona EM (1948) Las especies argentinas del género Polygala. Darwiniana 8 (2-3): 279-405.

Guaglianone ER (1972) Sinopsis de las especies de Ipheion Raf. y
Nothoscordum Kunth (Liliaceae) de Entre Ríos y regiones vecinas. Darwiniana 17: 159-242.

Hackel E, Arechavaleta J (1896) Danthonia montevidensis. Anales del Museo Nacional de Montevideo 1 (1-6): 369-370.

Hauman LL (1909) Cypella nova argentina. Apuntes de Historia Natural 1: 84-86.

Hauman LL (1917) Notas florísticas. Anales del Museo Nacional de Historia Natural de Buenos Aires 29: 391-444.

Haworth AH (1812) Synopsis plantarum succulentarum. Taylor et Socii, London, 207 pp. https://doi.org/10.5962/bhl.title.9462

H.B.K. [Humboldt F, Bonpland A, Kunth KS] (1823) Nova Genera et Species Plantarum (4th edition) 6. Librariae Graeco-LatinoGermanico, Paris, 542 pp., tab. 513-600. https://doi.org/10.5962/ bhl.title. 640

Hemsley WB (1881) Enumeration of the gamopetalae, with descriptions of new species. Biologia Centrali-Americana. Botany 2. R.H. Porter and Dulau \& Co., London, 576 pp. https://doi.org/10.5962/ bhl.title. 730

Herbert W (1840) Gelasine azurea. Azure Gelasine. Curtis's Botanical Magazine 66: pl. 3779.

Hieronymus GHEW (1880) Sertum patagonicum. Boletín de la Academia Nacional de Ciencias (Córdoba, Argentina) 3: 353-385.

Hieronymus GHEW (1897) Erster beitrag zug kenntnis der siphonogamenflora der Argentina und der angrenzender länder. Botanische Jahrbücher für Systematik, Pflanzengeschichte und Pflanzengeographie 22: 672-798.

Hildebrand FHG (1884) Die lebensverhältnisse der Oxalisarten. G. Fisher, Jena, $140 \mathrm{pp}$.

Hooker WJ (1826) Cypella herberthii. Curtis's Botanical Magazine 53: pl. 2637 (2nd p.).

Hooker WJ, Arnott GAW (1830-1841) Orden 21: Leguminosae. In: Hooker WJ, Beechey FW, Arnott GAW (Eds) The Botany of Captain Beechey's Voyage. H.G. Bohn, London, 16-23.

Hooker WJ, Arnott GAW (1833) Lathyrus crassipes. Botanical Miscellany 3: 198-199.

Hurrell JA (2009a) Flora rioplatense: sistemática, ecología y etnobotánica de las plantas vasculares rioplatenses: 3 (4). Editorial Lola, Buenos Aires, $422 \mathrm{pp}$

Hurrell JA (2009b) Alliaceae. In: Hurrel JA (Ed.). Flora rioplatense: sistemática, ecología y etnobotánica de las plantas vasculares rioplatenses 3 (4). Editorial Lola, Buenos Aires, 35-62.

Hurrell JA (2013) Flora Rioplatense: sistemática, ecología y etnobotánica de las plantas vasculares rioplatenses 2(7). Sociedad Argentina de Botánica, Buenos Aires, 304 pp.

INTA (2016) Instituto de Clima y Agua. http://inta.gob.ar/instdeclimayagua. Accessed on: 2016-12-09.

Isla FI, Cortizo LC, Turno Orellano HA (2001) Dinámica y evolución de las barreras medanosas, Provincia de Buenos Aires, Argentina. Revista Brasileira de Geomorfología 2 (1): 73-83.

Klotzsch JF (1852) Beiträge zu einer flora der Aequinoctial-Gegenden der Neuen Welt. Linnaea 25: 268-292.

Krebs JR, Wilson JD, Bradbury RB, Siriwardena GM (1999) The second Silent Spring? Nature 400: 611-612. https://doi.org/10.1038/23127

Kristensen MJ, Lavornia J, Leber V, Pose MP, Dellapé P, Salle A, Braccalente L, Giarratano M, Higuera M (2014) Estudios para la conservación de la pampa austral. I. Diagnóstico de la biodiversidad local. Revista Estudios Ambientales. 2(1): 105-117.

Kunth KS (1823) Voyage de Humboldt et Bonpland. Sixième Partie. Botanique. Nova Genera et Species Plantarum (4th ed.) Tome 6. Gilde Fils, Paris, 542 pp, pls 513-600. https://doi.org/10.5962/bhl. title. 640

Kunth KS (1843) Enumeratio plantarum omniumh hucusque cognitarum: secundum familias naturales disposita, adjectis characteribus, differentiis et synonymis 4. JG Cottae, Stutgardiae et Tubingae, 752 pp. https://doi.org/10.5962/bhl.title.67381

Kuntze CEO (1898) Revisio Generum Plantarum 3 (1-3) A. Felix, Leipzig. https://doi.org/10.5962/bhl.title.327

Lamarck JB (1786) Encyclopédie Méthodique. Botanique 2. Panckoucke, 
Paris, 774 pp. https://doi.org/10.5962/bhl.title.824

Landrum LR (2003) A revision of the Psidium salutare complex (Myrtaceae). Sida 20(4): 1463-1469.

Langsdorff GH, Fischer FEL (1810) Plantes recueillies pendant le voyage des Russes autour du monde. JG Cotta, Tubingen, 99 pp.

Lessing CF (1832) Synopsis generum compositarum 8. Dunckeri et Humblotii, Berolini, 473 pp. https://doi.org/10.5962/bhl.title.51470

Lindley J (1840) The Genera and Species of Orchidaceous Plants. Ridgways, London, 554 pp. https://doi.org/10.5962/bhl.title.499

Linnaeus C (1759) Systema Naturae per regna tria naturae, secundun classes, ordines, genera, species, cum characteribus, differenntiis, synonymus, locis (editio decima) 2 Laurentii Salvii. Holmiae, 825-1381. https://doi.org/10.5962/bhl.title.542

Linnaeus C (1767) Mantissa Plantarum: generum editionis VI. et specierum editionis II. Laurentii Salvii, Holmiae, 584 pp. https:// doi.org/10.5962/bhl.title.69083

Long MA, Grassini CM (1997) Actualización del conocimiento florístico del Parque Provincial Ernesto Tornquist. Informe final convenio de colaboración recíproca del Ministerio de Asuntos Agrarios de la Provincia de Buenos Aires. Universidad Nacional del Sur, Bahía Blanca. 257 pp.

Lorentz PG, Niederlein G (1881) Expedición al Rio Negro. Enumeración sistemática de las plantas colectadas durante la expedición. 2. Botánica. Ostwald and Martínez, Buenos Aires, 122 pp.

Lourteig A (2000) Oxalis L. subgéneros Monoxalis (Small) Lourt. Oxalis y Trifidus Lourt. Bradea 7 (2): 201-629.

Malme GOA (1904) Die Umbelliferen der sweiten Regnellschen Reise. Arkiv för Botanik 3 (13): 1-22.

Martius CFP (1884) Compositae. Flora Brasiliensis 6 (3). Frid. Fleische in Comm, Leipzig, 442 pp, 108 pls. https://doi.org/10.5962/bhl. title. 454

Martius CFP (1888) Rubiaceae. Flora Brasiliensis 6 (6). Frid. Fleischer in Comm, Leipzig, 466 pp, pls. 63-151. https://doi.org/10.5962/ bhl.title. 454

Mazzola MB, Kin AG, Morici EF, Babinec FJ, Tamborini G (2008) Efecto del gradiente altitudinal sobre la vegetación de las sierras de Lihuel Calel (La Pampa, Argentina). Boletín de la Sociedad Argentina de Botánica 43 (1-2): 103-119.

MEA [Millenium Ecosystems Assesment] (2005) Ecosystems and Human Well-Being: synthesis. Island Press, Washington DC, 137 pp

Méndez E (2009) Biodiversidad de la flora del flanco oriental de Cordón del Plata (Luján de Cuyo, Mendoza, Argentina). Catálogo florístico. Boletín de la Sociedad Argentina de Botánica 44(1-2): $75-102$.

Mez CC (1916) Bromeliaceae. Repertorium Specierum Novarum Regni Vegetabilis 14: 103-104.

Mittler L (1844) Auf neue erfahrungen gestüsste kultur und uebersicht der im teutschen handel vorkommenden cactuspflanzen 2. Ludwig Schreck, Leipzig.

Monserrat A (2010) Conservación en médanos: la vegetación de la costa bonaerense en Coronel Dorrego, Monte Hermoso y Coronel Rosales In: Isla FA, Lastra CA (Eds.). Manual de manejo de barreras medanosas de la Provincia de Buenos Aires. Mar del Plata, Ed. Eudem, Universidad Nacional de Mar del Plata, pp. 197-216.

Montes L, Alonso SI, Nuciari MC, Clausen AM, Guma IR and Echarte AM (2007) Flora espontánea del sudeste bonaerense, (2a ed). Unidad Integrada Balcarce: Estación Experimental Agropecuaria Balcarce Instituto Nacional de Tecnología Agropecuaria/Facultad Ciencias Agrarias Universidad Nacional de Mar del Plata, Balcarce, $102 \mathrm{pp}$.

Morici EA, Prina A, Alfonso GL, Muiño W (2010) Flora y vegetación del valle superior del Río Atuel (Mendoza-Argentina). Boletín de la Sociedad Argentina de Botánica 45 (1-2): 109-118.

Múlgura ME, O'Leary N, Rotman AD (2012) Flora Argentina. Flora Vascular de la República Argentina 14. Dicotyledoneae, Verbenaceae. Instituto de Botánica Darwinion-Instituto Multidisciplinario de Biología Vegetal, Consejo Nacional de Investigaciones Científicas y Técnicas, Córdoba, 230 pp.
Oggero AJ, Arana MD (2012) Inventario de las plantas vasculares del sur de la zona serrana de Córdoba, Argentina. Hoehnea 39(2): 171-199.

OPDS [Organismo Provincial de Desarrollo Sostenible de la Provincia de Buenos Aires] (2011) Plan de manejo ambiental. Ley 14.126, Partido de Tandil. http://www.gob.gba.gov.ar/legislacion/legislacion/opds-11-17.html. Accessed on 2016-10-4.

Papp C (1928) Einige neue Formen von Melica aus Südamerika. Notizblatt des Botanischen Gartens und Museums zu Berlin-Dahlem 10 (94): 352-358.

Parodi LR (1943) La vegetación del Departamento de San Martín en Corrientes (Argentina). Darwiniana 6: 127-178.

Paruelo JM, Guerschman JP, Verón SR (2005) Expansión agrícola y cambios en el uso del suelo. Ciencia Hoy 15 (87): 14-23.

Pilger RKF (1928) Die Gattung Plantago in Zentral- und Südamerika. Botanische Jahrbücher für Systematik, Pflanzengeschichte und Pflanzengeographie 62: 1-112.

Poiret JLM (1814) Lysimachia serpyllifolia. Encyclopédie Méthodique, Botanique, Supplement 3 (2): 477-478. https://doi.org/10.5962/ bhl.title. 826

Presl C B (1836) Filicaceae. Tentamen Pteridographiae, seu genera filicacearum praesertim juxta venarum decursum et distributionem exposita. Typis Filiorum Theophili Haase, Pragae, 290 pp. https:/ doi.org/10.5962/bhl.title.630

Rahn K (1983) Plantago ser. Brasilienses, a taxonomic revision. Nordic Journal of Botany 3: 331-342. https://doi.org/10.1111/ j.1756-1051.1981.tb00699.x

RARNAP [Red Argentina de Reservas Naturales Privadas] (2016) http://reservasprivadas.org.ar/reservas-naturales-privadas. Accessed on: 2016-5-24.

Raunkiaer C (1934) The life form of plants and statistical plant geography. Clarendon Press, Oxford, 632 pp

Ravenna P (1965) Notas sobre Iridáceas II. Boletín de la Sociedad Argentina de Botánica 10 (4): 311-322.

Ravenna P (1978). Studies in the Alliaceae II. Plant Life 34: 130-151.

Ravenna P (1988). A previous valid specific epithet for Gelasine azurea (Iridaceae). Phytologia 65 (2): 89-168.

Reichenbach HG (1878) Otia Botanica Hamburgensia. TT Meissneri, Hamburgi. 119 pp.

Roitman G, Hurrell JA (2009) Sisyrinchium. In: Hurrell JA (Ed.). Flora rioplatense: sistemática, ecología y etnobotánica de las plantas vasculares rioplatenses 3 (4). Ed. Lola, Buenos Aires, 273-289.

Rúgolo de Agrasar ZE (1982) Revalidación del género Bromidium Nees et Meyen emend. Pilger (Gramineae). Darwiniana 24: 187-216.

Saint Hilaire A (1822) Aperçu d'un voyage dans l'ínterieur du Brésil. Mémoires du Muséum d'Histoire Naturelle 9: 337-380.

Saint Hilaire A, de Jussieu A, Cambèssedes J (1829) Flora Brasiliae Meridionalis, 2. A. Belin, Bibliopolan, Paris, 381 pp., pls 83-158.

Saint Hilaire A, Naudin CH (1842) Flore du Brésil. Annales des Sciences Naturelles, Botanique (sér. 2) 18: 24-54.

(Eds) Sanhueza C, Zalba S (2014). Banco de semillas, germinación y longevidad de semillas de retama (Spartium junceum, Fabaceae): implicancias para su control. Boletín de la Sociedad Argentina de Botánica 49 (1): 67-76.

Sentinel Playground. 2017. Sinergise Ltd. http://apps.sentinel-hub.com/ sentinel-playground. Accessed on: 2017-11-6.

Sims J (1825) Plantago brasiliensis. Brazil plantain. Curtis's Botanical Magazine 53: pl. 2607.

Sleumer HO (1956) Die Hieracium Argentiniens unter Berücksichtigung der Nachbarländer. Botanische Jahrbücher für Systematik, Pflanzengeschichte und Pflanzengeographie 77 (1): 85-148.

Spegazzini C (1896) Contribución al estudio de la flora de la Sierra de la Ventana. Talleres de publicaciones del Museo, La Plata, 87 pp.

Spegazzini CL (1901a) Contribución al estudio de la Flora de Tandil. Sesé, Larrañaga y Renovales, La Plata, $60 \mathrm{pp}$.

Spegazzini CL (1901b) Flórula de ciudad de La Plata y su partido. Boletín de la Oficina Agricola Ganadera de la Provincia de Buenos Aires, La Plata 1: 235-245. 
Spegazzini CL (1925) Nuevas notas cactológicas. Anales de la Sociedad Científica Argentina 99: 85-156.

Sprengel CPJ (1825a) Systema Vegetabilium (16th edition) 1. Librariae Dieterichianae, Gottingae, 992 pp. https://doi.org/10.5962/bhl. title. 822

Sprengel CPJ (1825b). Systema Vegetabilium (16th edition) 2. Librariae Dieterichianae, Gottingae, 939 pp. https://doi.org/10.5962/bhl. title. 822

Sprengel CPJ (1826) Systema Vegetabilium (16th edition) 3. Librariae Dieterichianae, Gottingae, 935 pp. https://doi.org/10.5962/bhl. title. 822

Teruggi ME, Kilmurray, JO (1980) Sierras septentrionales de la Provincia de Buenos Aires. In: Turner J. (Ed.). Geología Regional Argentina II. Academia Nacional de Ciencias, Córdoba, 919-965

The Angiosperm Phylogeny Group (2016) An update of the Angiosperm Phylogeny Group classification for the orders and families of flowering plants: APG IV. Botanical Journal of the Linnean Society 181: 1-20. https://doi.org/10.1111/boj.12385

Torres MA (1968) Una nueva especie del género Melica. Boletín de la Sociedad Argentina de Botánica 12: 202-205.

Tortosa R (1995) Rhamnaceae. Flora Fanerogámica Argentina 9. Córdoba: PROFLORA, Consejo Nacional de Investigaciones Científicas y técnicas, Córdoba, 18 pp.

Troncoso NS (1964) Dilucidación de las especies platenses de Glandularia (Verbenaceae) de hojas disectas. Darwiniana 13 (2-4) $468-485$.

Tropicos (2017) Tropicos.org. Missouri Botanical Garden. http://www tropicos.org. Accessed on: 2017-11-6.

Tscharntke T, Klein AM, Kruess A, Steffan-Dewenter I, Thies C (2005) Landscape perspectives on agricultural intensification and biodiversity-ecosystem service management. Ecology Letters 8 (8): 857-874. https://doi.org/10.1111/j.1461-0248.2005.00782.x

Vahl C (1791) Om Perdicium og dens arter, og om en ny slaegt Rohria, henhorende til Compositas. Skrivter af Naturhistorie-Selskabet 2. Trykt hos N. Møller og søn, Kiobenhavn, 176 pp., pl. 9.

Valicenti R, Escobar RM, Requesens E, Orfila E, Farina E, D’Alfonso C, Scaramuzzino R (2000) Relaciones entre la vegetación y la fisiografía en una transección perpendicular al arroyo Azul (Provincia de Buenos Aires). Revista de la Facultad de Agronomía de La Pampa 11 (1): 31-38.
Valicenti R, Farina E, D'Alfonso C, Scaramuzzino R (2005) Caracterización fitosociológica de un pajonal serrano de Paspalum quadrifarium Lam. en Azul (Provincia de Buenos Aires). Revista Científica Agropecuaria 9 (2): 141-152.

Vega L, Bellagamba P (1990) Lista comentada de la herpetofauna de las sierras de Balcarce y Mar del Plata, Buenos Aires, Argentina. Cuadernos de Herpetologia 5 (2): 10-14.

Viglizzo EF, Frank FC, Carreño LV, Jobbágy EG, Pereyra H, Clatt J, Pincén D, Ricard MF (2011) Ecological and environmental footprint of 50 years of agricultural expansion in Argentina. Global Change Biology 17(2): 959-973. https://doi.org/10.1111/j.13652486.2010.02293.x

Willdenow CL (1809) Enumeratio Plantarum Horti Botanici Berolinensis 1. Taberna Libraria scholae Realis, Berolini, 1139 pp.

Zalba SM, Villamil, CB (2002). Woody plant invasion in relictual grasslands. Biological Invasions 4(1): 55-72. https://doi. org/10.1023/A:1020532609792

Zuloaga FO, Morrone O (1996) Catálogo de las plantas vasculares de la República Argentina. I. Pteridophyta, Gymnospermae y Angiospermae (Monocotyledonae excluyendo Poaceae). Monographs in Systematic Botany from the Missouri Botanical Garden 60. Missouri Botanical Garden, St. Louis, 332 pp.

Zuloaga FO, Morrone O (1999) Catálogo de las plantas vasculares de la República Argentina II. Dicotyledoneae. Monographs in Systematic Botany from the Missouri Botanical Garden 74. Missouri Botanical Garden, St. Louis, 246 pp.

Zuloaga FO, Anton AM, Rúgolo Z (2012a) Flora Argentina. Flora Vascular de la República Argentina 3 (1). Gráficamente Ediciones, Córdoba, $588 \mathrm{pp}$.

Zuloaga FO, Anton AM, Rúgolo Z (2012b) Flora Argentina. Flora Vascular de la República Argentina 3 (2). Gráficamente Ediciones, Córdoba, $523 \mathrm{pp}$.

Zuloaga FO, Belgrano M, Antón AM (2014a). Flora Argentina. Flora Vascular de la República Argentina 7 (1). Instituto de Botánica Darwinion, Consejo Nacional de Investigaciones Científicas y Técnicas, Córdoba, 546 pp.

Zuloaga FO, Belgrano M, Antón AM (2014b) Flora Argentina. Flora Vascular de la República Argentina 7 (3). Instituto de Botánica Darwinion, Consejo Nacional de Investigaciones Científicas y Técnicas, Córdoba, 306 pp. 